\title{
ZERO-TEMPERATURE 2D STOCHASTIC ISING MODEL AND ANISOTROPIC CURVE-SHORTENING FLOW
}

\author{
HUBERT LACOIN, FRANÇOIS SIMENHAUS, AND FABIO LUCIO TONINELLI
}

\begin{abstract}
Let $\mathcal{D}$ be a simply connected, smooth enough domain of $\mathbb{R}^{2}$. For $L>0$ consider the continuous time, zero-temperature heat bath dynamics for the nearest-neighbor Ising model on $\mathbb{Z}^{2}$ with initial condition such that $\sigma_{x}=-1$ if $x \in L \mathcal{D}$ and $\sigma_{x}=+1$ otherwise. It is conjectured [24] that, in the diffusive limit where space is rescaled by $L$, time by $L^{2}$ and $L \rightarrow \infty$, the boundary of the droplet of "-" spins follows a deterministic anisotropic curve-shortening flow, where the normal velocity at a point of its boundary is given by the local curvature times an explicit function of the local slope. The behavior should be similar at finite temperature $T<T_{c}$, with a different temperature-dependent anisotropy function.

We prove this conjecture (at zero temperature) when $\mathcal{D}$ is convex. Existence and regularity of the solution of the deterministic curve-shortening flow is not obvious a priori and is part of our result. To our knowledge, this is the first proof of mean curvature-type droplet shrinking for a model with genuine microscopic dynamics.
\end{abstract}

2000 Mathematics Subject Classification: 60K35, 82C20

Keywords: Ising model, Glauber dynamics, Curve-shortening flow.

\section{Contents}

1. Introduction 1

2. Model and results 3

3. Local interface dynamics $\quad 8$

4. Proof of Theorem 2.3: evolution of the scale-invariant droplet 13

5. Proof of Theorem 2.1: existence of anisotropic curve-shortening flow with convex initial condition 26

6. Proof of Theorem 2.2: evolution of a convex droplet 32

7. Proof of Theorem 3.2: scaling limit for SSEP 38

Appendix A. Proof of Theorem 3.4: scaling limit for the zero-range process 40

Acknowledgments $\quad 51$

$\begin{array}{ll}\text { References } & 51\end{array}$

\section{INTRODUCTION}

Consider a thermodynamic system with two coexisting phases and imagine to prepare it in an initial condition where a droplet of one phase is immersed in the other phase. If the system undergoes a dynamics that does not conserve the order parameter, it is well understood phenomenologically [20] that the droplet will shrink in order to decrease its surface tension until it eventually disappears, and that (roughly speaking) the normal speed at a point of its

F. T. was partially supported by European Research Council through the "Advanced Grant" PTRELSS 228032 and by ANR project SHEPI. 
boundary will be proportional to the local mean curvature. Deriving such behavior from first principles, i.e. from a microscopic model undergoing a local (stochastic) dynamics, is a much harder task and this program was started only rather recently [24]. More precisely, what one expects is that, if the initial droplet is of diameter $L$, it will "disappear" within a time of order $L^{2}$ (this behavior is sometimes referred to as "Lifshitz law"). Moreover, in the "diffusive limit" where $L \rightarrow \infty$ and at the same time space is rescaled by $L$ (so that the initial droplet is of size $O(1))$ and time is accelerated by $L^{2}$, the droplet evolution should become deterministic and follow some anisotropic version of a mean curvature flow. Anisotropy (i.e. the fact that the normal velocity will also depend on the local orientation of the droplet boundary) is expected when the underlying model is defined on a lattice, as will be the case for us.

Up to now, mathematical progress on this issue has been rather modest, the main difficulty being that it is not clear how to implement the idea that the fast modes related to relaxation inside the two pure phases should decouple from slow modes related to the interface motion, which are responsible for the diffusive time scaling $L^{2}$.

A fairly well understood situation is that where the interface can be described by a height function and the bulk structure of the two phases is disregarded: this is possible (by definition) for the so-called "effective interface models" or Ginzburg-Landau $\nabla \phi$ interface models: for models with continuous heights and strictly convex potential undergoing a Langevin-type dynamics, Funaki and Spohn [10] derived the full mean-curvature motion in the diffusive scaling. Another well-studied case is that of models with Kac-type potentials: in this case, mean-curvature motion can be proven to emerge $[5,6,15]$ in a limit where interaction range is taken to infinity at some stage, but in this limit there is no sharp interface separating the phases and the system becomes very close to mean-field.

As for true lattice models, results are much more scarce. For instance, for the two-dimensional nearest-neighbor Ising model below the critical temperature, the best known upper bound on the "disappearance time" for a droplet of "- phase" immersed in the "+ phase" is of the order $L^{c(T)} \log L[22]$, very far from the expected $L^{2}$ scaling. Recently, a weak version of the Lifshitz law was proven for the three-dimensional Ising model at zero temperature: the disappearance time of a "-" droplet is of order $L^{2}$ (upper and lower bounds), up to multiplicative logarithmic (in $L$ ) corrections [2]. When the dimension is higher than three (always at zero temperature), an upper bound for the disappearance time of order $L^{2}(\log L)^{c}$, for some constant $c$, was proven in [18].

In this work, we concentrate on the two-dimensional nearest-neighbor Ising model on the infinite square lattice. The dynamics takes a very simple form: each spin is updated with rate one and after the update it takes the same value of the majority of its neighbors, or the value \pm 1 with equal probabilities if exactly two neighbors are "+" and two are "-". In this case, the disappearance time of a large "-" droplet should be asymptotically given by one half its volume (number of "-" spins). Moreover, in the diffusive scaling limit the droplet boundary should be given by a deterministic curve $\gamma(t)$ whose normal speed is given by the local (signed) curvature, times a function $a(\theta)$ where $\theta$ is the angle of the local normal vector. The function $a(\cdot)$ is explicitly known, see $(2.4)$. In this two-dimensional setting, it is more natural to refer to such flow as "(anisotropic) curve-shortening flow" (rather than "mean curvature flow").

Our main result (Theorem 2.2) is a proof of the curve-shortening conjecture (and, as a byproduct, of the Lifshitz law) when the initial droplet is convex.

There are some previous partial results available on this problem. In [23] (cf. also [21]), Rost described (with the language of exclusion processes rather than spin systems) the scaling limit of the evolution when initially spins are "-" in the first quadrant of $\mathbb{Z}^{2}$ (infinite corner) and "+" elsewhere. This is a simple situation because the interface motion is mapped to symmetric 
simple exclusion and is described by the associated height function at all times. In [24], Spohn described the scaling limit of the interface motion in a situation that more or less corresponds to the zero-temperature Ising model in an infinite vertical cylinder, with an initial condition such that the interface separating "+" from "-" spins can be globally described by a height function at all times (in particular, this cannot describe a droplet, and implicitly he has to modify the dynamics to guarantee that droplets do not appear later in the evolution). In [4], Chayes et al. proved the Lifshitz law (but not the curve-shortening conjecture) for a modified dynamics where updates which break the droplet into several droplets are forbidden. In [3], Cerf and Louhichi computed the "drift at time 0" of the droplet (for the non-modified dynamics), but their result does not allow to get information on the evolution for finite time $t>0$.

An important building block of our proof of the anisotropic curve-shortening conjecture is that, as was well understood by Spohn in [24], locally the interface can be (roughly speaking) described by the hydrodynamic limit of a certain zero-range process at the points where the tangent to the boundary is horizontal or vertical, and by the hydrodynamic limit of the symmetric simple exclusion process elsewhere. However, such correspondence is not exact due to updates that split the droplet into more than one connected components (see for instance Figure 4). In other words, the interface is not (even locally) the graph of a function. Also, it is a non-trivial task to patch together the various pieces of "local analysis" to control globally the evolution of the droplet. Both problems will be tamed by a sequence of monotonicity arguments, which are allowed because the dynamics conserves the stochastic ordering among configurations.

In order to prove Theorem 2.2, we also need to know that a classical solution to the anisotropic curve-shortening flow exists up to the time where the droplet disappears, and (crucially) that such solution is sufficiently regular in space and time (i.e. that the curvature is a Lipschitz function of the angle and a continous function of time). To our surprise, we found that the existing literature on curve-shortening flows does not provide global (in time) results for the flow associated to the zero-temperature $2 \mathrm{~d}$ Ising model. The reason is that the anisotropy function $a(\cdot)$ is not smooth (its derivative has jumps, reflecting the singularities of the surface tension at zero temperature), while the existing results assume that $a(\cdot)$ is at least $C^{2}$, cf. $[11,12$. To prove existence, uniqueness and regularity of the solutions (cf. Theorem 2.1), we will regularize the function $a(\cdot)$ and then analyze the regularized flow following the ideas of $[11,12]$. Of course, it will be crucial to guarantee that all the estimates we need are uniform in the regularization parameter, which tends to zero in the end.

The case where the initial "-" droplet is non-convex will be considered in future work. The additional difficulties are two-fold. First of all, from the analytic point of view, available global existence and regularity results for the solution of curve-shortening flows with non-convex initial condition seem to be limited to the isotropic case where $a(\cdot) \equiv 1$ [14]. Secondly, due to the fact that the droplet will move at the same time outwards and inwards at different locations according to the sign of the curvature, various monotonicity arguments we use in the following will not work.

\section{Model AND RESUlts}

2.1. Glauber dynamics and expected limiting evolution. Set $\mathbb{Z}^{*}:=\mathbb{Z}+\frac{1}{2}:=\{x+$ $(1 / 2) \mid x \in \mathbb{Z}\}$. We consider the zero-temperature stochastic Ising model on $\left(\mathbb{Z}^{*}\right)^{2}$ with its usual lattice structure ( $x$ and $y$ are linked if $|x-y|=1$ for the $l_{1}$ norm). Is is a continuous time Markov chain $(\sigma(t))_{t>0}$ on the space of spin configurations on $\left(\mathbb{Z}^{*}\right)^{2}, \Omega:=\{-1,1\}^{\left(\mathbb{Z}^{*}\right)^{2}}$. We write $\sigma(t)=\left(\sigma_{x}(t)\right)_{x \in\left(\mathbb{Z}^{*}\right)^{2}}$ and for simplicity we write $\sigma_{x}=-\left(\right.$ resp. $\sigma_{x}=+$ ) instead of $\sigma_{x}=-1$ (resp. $\left.\sigma_{x}=+1\right)$. 
The transition rules are the following : for each site $x \in\left(\mathbb{Z}^{*}\right)^{2}$, the value $\sigma_{x}$ of the spin at $x$ is updated independently with rate one. When the spin at a site is updated, it takes the same value as the spin of the majority of its neighbors, or the values \pm 1 with equal probabilities $1 / 2$ if two neighbors have " +" spins and the other two "-" spins. That these rules yield a well-defined Markov chain even in infinite volume is a standard fact (cf. [21]). In the following (cf. (2.1)), we will consider only initial conditions where the number of "-" spins is finite. It is easy to realize that the spins outside the smallest square containing all the initial "-" spins stay "+" forever, so that in reality we have a dynamics on a finite volume and the question of existence of the process is trivial.

We are interested in the evolution of the set of "-" spins for this Markov chain when the initial condition $\sigma(0)$ is a large droplet, i.e. a finite connected set of " -" spins surrounded by " +" spins. In that case, almost surely, after a finite time $\tau_{+}$, all the "-" spins have turned to "+" and the dynamics will stay forever in the all "+" configuration (which is an absorbing state). Our aim is to describe the evolution of the shape of the rescaled minus droplet on a proper (diffusive) time-scale. In the next paragraph we make that aim more precise.

We consider a compact, simply connected subset $\mathcal{D} \subset[-1,1]^{2}$ whose boundary is a closed smooth curve. Given $L \in \mathbb{N}$ we consider the Markov chain described above with initial condition

$$
\sigma_{x}(0)= \begin{cases}-1 & \text { if } x \in\left(\mathbb{Z}^{*}\right)^{2} \cap L \mathcal{D}, \\ +1 & \text { otherwise. }\end{cases}
$$

In order to see a set of "-" spins as a subset of $\mathbb{R}^{2}$, each vertex $x \in\left(\mathbb{Z}^{*}\right)^{2}$ may be identified with the closed square of side-length one centered at $x$,

$$
\mathcal{C}_{x}:=x+[-1 / 2,1 / 2]^{2} .
$$

One defines

$$
\mathcal{A}_{L}(t):=\bigcup_{\left\{x: \sigma_{x}(t)=-1\right\}} \mathcal{C}_{x}
$$

which is the "- droplet" at time $t$ for the dynamics. The boundary of $\mathcal{A}_{L}(t)$ is a union of edges of $\mathbb{Z}^{2}$ (this is the only reason why we defined the Ising model on $\overline{\mathbb{Z}}^{2}$ ).

What was conjectured by Lifshitz [20] on heuristic grounds for the low temperature Ising model is that $\mathcal{A}_{L}(t)$ should follow an anisotropic curve shortening motion: after rescaling space by $L$ and time by $L^{2}$ and letting $L$ tend to infinity, the motion of the interface between $\mathcal{A}_{L}(t)$ and its complement should be deterministic and the local drift of the interface should be proportional to the curvature, with an anisotropic correction to reflect anisotropy of the underlying lattice. More precisely, one can formulate this conjecture as follows [24]: Let $\gamma(t, L)$ denote the boundary of the (random) set $(1 / L) \mathcal{A}_{L}\left(L^{2} t\right)$. Then, for $L \rightarrow \infty, \gamma(t, L)$ should converge to a deterministic curve $\gamma(t)$ and the evolution of $(\gamma(t))_{t \geq 0}$ should be such that the normal velocity at a point $x \in \gamma(t)$ is given by the curvature at $x$, times an anisotropic factor $a\left(\theta_{x}\right)$, where $\theta_{x}$ is the slope of the outwards directed normal to $\gamma(t)$ at $x$. The velocity is directed inwards at points where $\gamma(t)$ is convex and outwards at points where it is concave. The function $a(\cdot)$ should have the explicit expression

$$
a(\theta):=\frac{1}{2(|\cos (\theta)|+|\sin (\theta)|)^{2}} .
$$

In particular, the curve $\gamma(t)$ should shrink to a point in a finite time

$$
t_{0}=\frac{\operatorname{Area}(\mathcal{D})}{\int_{0}^{2 \pi} a(\theta) \mathrm{d} \theta}=\frac{\operatorname{Area}(\mathcal{D})}{2} .
$$


Note that the function $a(\cdot)$ is symmetric around 0 and is periodic with period $\pi / 2$, which reflects the discrete symmetries of the lattice $\overline{\mathbb{Z}}^{2}$. It is important to note for the following that $a(\cdot)$ is $C^{\infty}$ except at $\theta=j \pi / 2, j=0, \ldots, 3$ where it is only continuous and its first derivative has a jump: indeed, $a(\theta) \sim 1 / 2-|\theta-i \pi / 2|$ for $\theta$ close to $i \pi / 2, i=0, \ldots, 3$.

\subsection{Results.}

2.2.1. Convex initial droplet. We prove the anisotropic curve shortening conjecture in the case where the initial droplet is convex and suitably smooth. Given a strictly convex smooth curve $\gamma=\partial \mathcal{D}$ in $\mathbb{R}^{2}$, we parameterize it following a standard convention of convex geometry (cf. e.g. [12] and Figure 1). For $\theta \in[0,2 \pi]$ let $v(\theta)$ be the unit vector forming an anticlockwise angle $\theta$ with the horizontal axis and let

$$
h(\theta)=\sup \{x \cdot v(\theta), x \in \gamma\}
$$

with $\cdot$ the usual scalar product in $\mathbb{R}^{2}$.

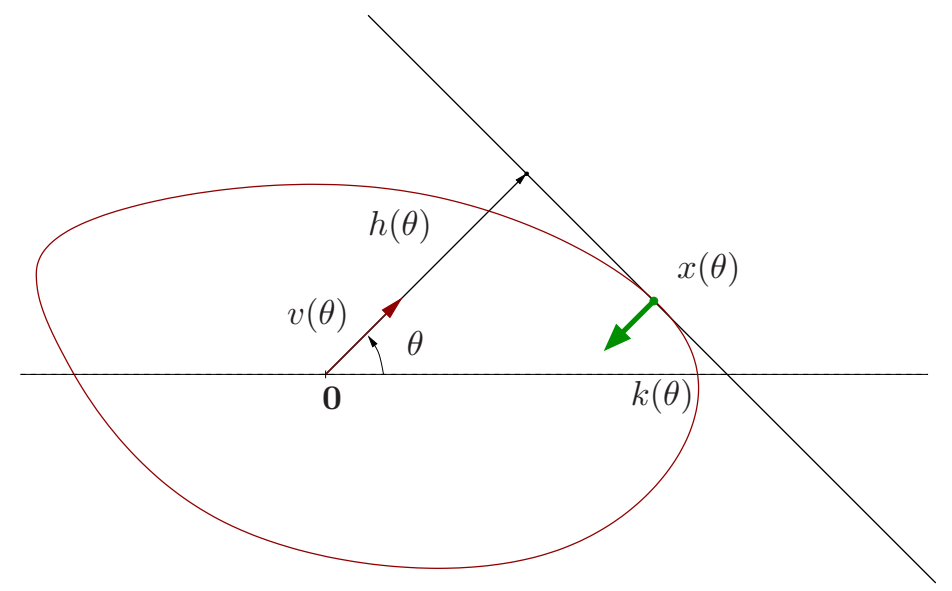

Figure 1. A graphic description of the support function $h$. Given $\theta$, consider the point $x(\theta)$ of $\gamma$ that maximizes $x \cdot v(\theta)$ (it is unique if the curve is strictly convex). Then $h(\theta)=x(\theta) \cdot v(\theta)$, and $k(\theta)$ is the norm of the curvature vector of $\gamma$ (bold vector) at $x(\theta)$. If the tangent to $\gamma$ at $x$ exists it is normal to $v(\theta)$ and $|h(\theta)|$ is the distance between the tangent and the origin.

The function $\theta \mapsto h(\theta)$ (called "the support function") uniquely determines $\gamma$ :

$$
\mathcal{D}=\cap_{0 \leq \theta \leq 2 \pi}\left\{x \in \mathbb{R}^{2}: x \cdot v(\theta) \leq h(\theta)\right\} .
$$

With this parameterization, the anisotropic curve shortening evolution reads

$$
\left\{\begin{array}{l}
\partial_{t} h(\theta, t)=-a(\theta) k(\theta, t) \\
h(\theta, 0)=h(\theta)
\end{array}\right.
$$

where, for a convex curve $\gamma, k(\theta) \geq 0$ is the curvature at the point $x(\theta) \in \gamma$ where the outward normal an anticlockwise angle $\theta$ with the horizontal axis and the $t$-derivative is taken at constant $\theta$ (see [12, Lemma 2.1] for a proof of $(2.7)$ ). Of course $h(\cdot)$ is the support function of $\partial \mathcal{D}$.

In general, even proving the existence of a solution of $(2.7)$ with $a(\cdot)$ given in $(2.4)$ is nontrivial, since $a(\cdot)$ has points of non-differentiability and the existing literature (e.g. $[11,12])$ usually assumes that $a(\cdot)$ is at least $C^{2}$. 
Our first result is

Theorem 2.1. Let $\mathcal{D} \subset[-1,1]^{2}$ be strictly convex and assume that its boundary $\gamma=\partial \mathcal{D}$ is a curve whose curvature $[0,2 \pi] \ni \theta \mapsto k(\theta)$ defines a positive, $2 \pi$-periodic, Lipschitz function. Then there exists a unique flow of convex curves $(\gamma(t))_{t}$ with curvature defined everywhere, such that $\gamma(0)=\gamma$ and that the corresponding support function $h(\theta, t)$ solves $(2.7)$ for $t \geq 0$ and satisfies the correct initial condition $h(\theta, 0)=h(\theta)$. The curve $\gamma(t)$ shrinks to a point $\mathbf{x}_{f} \in \mathbb{R}^{2}$ at time $t_{f}=\operatorname{Area}(\mathcal{D}) / 2$. For $t<t_{f}, \gamma(t)$ is a smooth curve in the following sense: its curvature function $k(\cdot, t)$ is Lipschitz and bounded away from 0 and infinity on any compact subset of $\left[0, t_{f}\right)$.

We let $\mathcal{D}(t)$ denote the convex closed set enclosed by $\gamma(t)$ (of course, $\mathcal{D}(t=0)=\mathcal{D}$ ). Also, we use the convention that $\mathcal{D}(t)=\left\{\mathbf{x}_{f}\right\}$ if $t \geq t_{f}$.

For $\delta>0$ let $B(x, \delta)$ denote the ball of radius $\delta$ centered at $x$ and for any compact set $\mathcal{C} \subset \mathbb{R}^{2}$ define

$$
\mathcal{C}^{(\delta)}:=\bigcup_{x \in \mathcal{C}} B(x, \delta), \quad \mathcal{C}^{(-\delta)}:=\left(\bigcup_{x \notin \mathcal{C}} B(x, \delta)\right)^{c} .
$$

Note that $\mathcal{D}(t)^{(\delta)}=B\left(\mathbf{x}_{f}, \delta\right)$ and $\mathcal{D}(t)^{(-\delta)}=\emptyset$ if $t \geq t_{f}$.

An event $B_{L}$ is said to occur with high probability (w.h.p.) if $\lim _{L \rightarrow \infty} P\left(B_{L}\right)=1$.

Theorem 2.2. Under the same assumptions on $\mathcal{D}$ as in Theorem 2.1, for any $\delta>0$ one has w.h.p.

$$
\begin{gathered}
\mathcal{D}^{(-\delta)}(t) \subset \frac{1}{L} \mathcal{A}_{L}\left(L^{2} t\right) \subset \mathcal{D}^{(\delta)}(t) \quad \text { for every } \quad 0 \leq t \leq t_{f}+\delta \\
\mathcal{A}_{L}\left(L^{2} t\right)=\emptyset \quad \text { for every } t>t_{f}+\delta .
\end{gathered}
$$

In particular, one has the following convergence in probability:

$$
\lim _{L \rightarrow \infty} \frac{\tau_{+}}{L^{2} \operatorname{Area}(\mathcal{D})}=\frac{1}{2} .
$$

The reason why in Theorems 2.1 and 2.2 we do not content ourselves with, say, initial $C^{\infty}$ curves is that, as we see in next section, there is a very natural initial condition whose curvature function is only Lipschitz and not $C^{1}$ (and stays so at later times).

Theorem 2.2 does not apply directly if one considers $\mathcal{D}=[0,1]^{2}$ or any other non-smooth convex set. However, approximating $\mathcal{D}$ from above and below by smooth compact sets and using monotonicity (cf. Section 2.3), one sees easily that (2.11) holds in any case. In particular, the disappearance time of an $L \times L$ square droplet is with high probability $L^{2} / 2(1+o(1))$.

Theorems 2.2 and 2.3 tell us that for our choices of initial configuration, the disappearance time of the minus droplet is non-random at first order. This implies that the variation distance of our Markov Chain from equilibrium (which is concentrated on the all-plus configuration) drops abruptly from 1 to 0 around time $L^{2} t_{f}$ within a time-window of width $o\left(L^{2}\right) \ll L^{2} t_{f}$ (we conjecture that the correct order of the window should be $O\left(L^{3 / 2}\right)$ ). This is a particular instance of a phenomenon called cut-off (cf. [7] and [19]).

2.2.2. Scale-invariant droplet. A particular case of Theorem 2.2 is that where the initial condition is scale invariant, i.e. when the limiting evolution $(\gamma(t))_{t}$ is a homothetic contraction. Consider the function

$$
f_{0}:\left[-\frac{1}{\sqrt{2}},+\frac{1}{\sqrt{2}}\right] \ni x \mapsto f_{0}(x)=\beta\left\{4 \alpha x \int_{0}^{x} e^{2 \alpha t^{2}} d t-e^{2 \alpha x^{2}}\right\},
$$


where $\alpha$ is the unique positive solution of

$$
4 \sqrt{2} \alpha e^{-\alpha} \int_{0}^{1 / \sqrt{2}} e^{2 \alpha t^{2}} d t=1
$$

and

$$
\beta=-\sqrt{2} e^{-\alpha}<0 .
$$

Note that $f_{0}$ is $\mathcal{C}^{\infty}$, positive, concave, symmetric around 0 and increasing on $\left[-\frac{1}{\sqrt{2}}, 0\right]$. We denote by $\left(\mathbf{e}_{1}, \mathbf{e}_{2}\right)$ the canonical basis of $\mathbb{R}^{2}$ and $\left(\mathbf{f}_{1}, \mathbf{f}_{2}\right)=\left(\frac{\mathbf{e}_{1}-\mathbf{e}_{2}}{\sqrt{2}}, \frac{\mathbf{e}_{1}+\mathbf{e}_{2}}{\sqrt{2}}\right)$ the image of $\left(\mathbf{e}_{1}, \mathbf{e}_{2}\right)$ by the rotation of angle $-\pi / 4$. We also define the curve $\gamma_{1}$ to be the graph of $f_{0}$ in the coordinate system $\left(\mathbf{f}_{1}, \mathbf{f}_{2}\right)$, i.e.

$$
\gamma_{1}:=\left\{x \mathbf{f}_{1}+f_{0}(x) \mathbf{f}_{2} \mid x \in\left[-\frac{1}{\sqrt{2}}, \frac{1}{\sqrt{2}}\right]\right\} .
$$

If $S_{1}$ (resp. $S_{2}$ ) denotes the symmetry with respect to the axis $\mathbf{e}_{1}$ (resp. $\mathbf{e}_{2}$ ) one defines the closed curve $\gamma$ by

$$
\gamma=\gamma_{1} \cup\left(S_{1} \gamma_{1}\right) \cup\left(S_{2} \gamma_{1}\right) \cup\left(\left(S_{1} \circ S_{2}\right) \gamma_{1}\right) .
$$

In the sequel $\mathscr{D}$ denotes the compact, convex set enclosed in $\gamma$, see Figure 2.

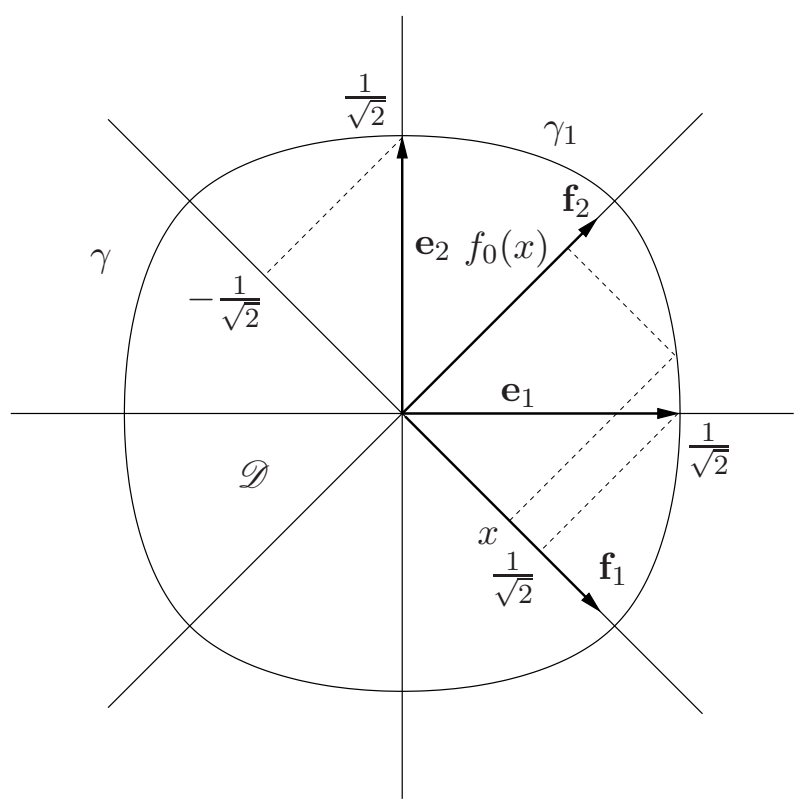

Figure 2. The curve $\gamma=\partial \mathscr{D}$ and the coordinate systems $\left(\mathbf{e}_{1}, \mathbf{e}_{2}\right)$ and $\left(\mathbf{f}_{1}, \mathbf{f}_{2}\right)$.

One can check that the curvature function $\theta \mapsto k(\theta)$ of $\partial \mathscr{D}$ is Lipschitz and bounded away from zero, but not differentiable at $\theta=i \pi / 2, i=0,1,2,3$. In this case, Theorem 2.2 can be formulated as follows.

Theorem 2.3. Assume that $\mathcal{D}=\mathscr{D}$. For any $\eta>0$, w.h.p,

$$
(\sqrt{1-2 \alpha t}-\eta) \mathscr{D} \subset \frac{1}{L} \mathcal{A}_{L}\left(t L^{2}\right) \subset(\sqrt{1-2 \alpha t}+\eta) \mathscr{D} \quad \text { for every } \quad t \geq 0
$$


where we work with the convention that $\sqrt{x}=0$ for $x \leq 0$ and that $x \mathscr{D}=\emptyset$ for $x<0$. Moreover, one has the following convergence in probability:

$$
\lim _{L \rightarrow \infty} \frac{\tau_{+}}{\operatorname{Area}(L \mathscr{D})}=\alpha \lim _{L \rightarrow \infty} \frac{\tau_{+}}{L^{2}}=\frac{1}{2} .
$$

It is easy to check, using Lemma 3.6 below and a couple of integrations by parts, that $\operatorname{Area}(\mathscr{D})=1 / \alpha$, yielding the first equality in (2.18). The expression (2.12) for the invariant shape appears also, although with different notations, in the recent work [17].

2.3. Graphical construction of the dynamics and monotonicity. Before starting the proofs, we wish to give a construction of the Markov process (called sometimes the graphical construction) that yields nice monotonicity properties. We consider a family of independent Poisson clock processes $\left(\tau^{x}\right)_{x \in \overline{\mathbb{Z}}^{2}}$. More precisely, to each site $x \in\left(\mathbb{Z}^{*}\right)^{2}$ one associates a random sequence (independently from other sites) of times $\left(\tau_{n}^{x}\right)_{n>0}$, that are such that $\tau_{0}^{x}=0$ and $\left(\tau_{n+1}^{x}-\tau_{n}^{x}\right)_{n \geq 0}$ are IID exponential variables with mean one. One also defines random variables $\left(U_{n, x}\right)_{n \geq 0}, x \in\left(\mathbb{Z}^{*}\right)^{2}$ that are IID Bernoulli variables of parameter $1 / 2$, with values \pm 1 .

Then given an initial configuration $\xi \in\{-1,1\}^{\left(\mathbb{Z}^{*}\right)^{2}}$ one constructs the dynamics $\sigma^{\xi}(t)$ starting from $\sigma^{\xi}(0)=\xi$ as follows

- $\left(\sigma_{x}(t)\right)_{t \geq 0}$ is constant on the intervals of the type $\left[\tau_{n}^{x}, \tau_{n+1}^{x}\right)$.

- $\sigma_{x}\left(\tau_{n}^{x}\right)$ is chosen to be equal to \pm 1 if a strict majority of the neighbors of $x$ satisfies $\sigma_{y}\left(\tau_{n}^{x}\right)= \pm 1$, and $U_{n, x}$ otherwise (this definition makes sense as, almost surely, two neighbors will not update at the same time unless with zero probability.)

This construction gives a simple way to define simultaneously the dynamics for all initial conditions (we denote by $P$ the associated probability). Moreover this construction preserves the natural order on $\{-1,+1\}^{\left(\mathbb{Z}^{*}\right)^{2}}$, given by

$$
\xi \geq \xi^{\prime} \Leftrightarrow \xi_{x} \geq \xi_{x}^{\prime} \text { for every } x \in\left(\mathbb{Z}^{*}\right)^{2}
$$

(this order is just the opposite of the inclusion order for the set of "-" spins, which is therefore also preserved). Indeed, if $\xi \geq \xi^{\prime}$, with the above construction, one has $P$-a.s.

$$
\forall t>0 \quad \sigma^{\xi}(t) \geq \sigma^{\xi^{\prime}}(t)
$$

\section{LOCAL INTERFACE DYNAMiCS}

One problem one has to deal with when proving mean curvature motion for the whole droplet is that even though initially the interface between "+" and "-" (i.e. the geometric boundary of the set $\mathcal{A}_{L}(0)$ ) is a simple curve, it can later split to form several loops. In fact, as a byproduct of our results, we will obtain that, with large probability, only very small extra loops can be created. We will tackle this problem by introducing some auxiliary dynamics that do not allow creation of new loops and stochastically compare to the original one.

A second problem is that the interface that one has to control is not exactly the graph of function, for which it would be easier to describe the macroscopic motion using partial differential equations. We begin by studying two dynamics for which the interface is indeed a graph, and which have locally the same large-scale behavior as the true evolution. It is more natural to introduce these dynamics as dynamics on interfaces rather than dynamics on spins. Our work then will consist of glueing together the "local results" of Theorems 3.2 and 3.4 to get Theorems 2.2 and 2.3 . 
3.1. Local dynamics away from the poles and simple exclusion process. The first auxiliary dynamics is used to control the evolution of the boundary of $(1 / L) \mathcal{A}_{L}\left(t L^{2}\right)$ away from the points (the poles) where the tangent to the deterministic curve $\gamma(t)$ is either horizontal or vertical. The evolution near the poles will be analyzed via a second auxiliary dynamics, see Section 3.2.

Given two positive natural numbers $M, N$ consider the state-space $\Omega_{M, N}$ of nearest-neighbor directed paths of length $L:=M+N$ with $M$ steps up and $N$ steps down:

$$
\Omega_{M, N}=\left\{\left(h_{x}\right)_{x \in\{0, \ldots, M+N\}} \in \mathbb{Z}^{M+N+1}|| h_{x+1}-h_{x} \mid=1, h_{0}=0 ; h_{M+N}=M-N\right\} .
$$

Given $h \in \Omega_{M, N}$ and $x \in\{1, \ldots, L-1\}$, we denote by $h^{(x)}$ the path with a corner "flipped" at $x$ defined by $h_{y}^{(x)}=h_{y}$ for all $y \neq x$ and

$$
h_{x}^{(x)}:=\left\{\begin{array}{l}
h_{x}-2 \text { if } h_{x \pm 1}=h_{x}-1, \\
h_{x}+2 \text { if } h_{x \pm 1}=h_{x}+1, \\
h_{x} \text { if }\left|h_{x+1}-h_{x}\right|=2 .
\end{array}\right.
$$

The dynamics $\Omega_{M, N}$ we consider is the one that flips every corner with rate $1 / 2$. More precisely it is the Markov chain whose generator $\mathcal{L}$ is defined as

$$
\mathcal{L} f(h):=\frac{1}{2} \sum_{x=1}^{L-1}\left(f\left(h^{(x)}\right)-f(h)\right), \quad \forall f: \Omega_{M, N} \mapsto \mathbb{R} .
$$

We denote by $(h(t))_{t \geq 0}$ the trajectory of the Markov chain started from initial condition $h(0):=h^{0} \in \Omega_{M, N}$.

Remark 3.1. Note that this dynamics is in one-to-one correspondence with the Ising dynamics on a rectangle $N \times M$ with "+" boundary conditions on two adjacent sides and "-" boundary conditions on the two opposite sides, provided that the initial configuration is such that the length of the $-/+$ boundary is $M+N$ (i.e. the minimal possible length). More precisely (see Figure 3) the correspondence is obtained by taking the graph of $h$, rotating it by $\pi / 4$ and rescaling space by a factor $\sqrt{2}$ (so that squares have side-length one on the right picture). Note that we are implicitly identifying an element $h \in \Omega_{M, N}$ with a continuous function $F$ : $[0, M+N] \mapsto \mathbb{R}$ such that $F(x)=h_{x}$ for $x=0,1, \ldots, M+N$ and $F(\cdot)$ is linear on intervals $(n, n+1)$ with integer $n$.

This corner-flip dynamics has been widely studied (see e.g. [25]) and can be mapped to the symmetric simple exclusion process (SSEP) on a finite interval (just say that there is a particle at $x=0, \ldots, M+N-1$ if and only if $h_{x+1}-h_{x}=+1$, and check that dynamics in terms of particles coincides with that of SSEP). From hydrodynamic-limit results, it is quite clear that the rescaled version of $h$ when $M, N$ tends to infinity should satisfy the heat equation (see [16, Section 4.2.] for an account on hydrodynamic equations for the exclusion process). However, we have not found in the literature a proof of the following precise statement we need (we give a concise proof of it in Section 7):

Theorem 3.2. Given a 1-Lipschitz function $\phi^{0}:[0,1] \mapsto \mathbb{R}$ with $\phi^{0}(0)=0$, let $(h(t))_{t \geq 0}$ the dynamics starting from initial condition $h^{0} \in \Omega_{M_{L}, N_{L}}$ given by

$$
\begin{aligned}
& h_{x}^{0}:=2\left\lfloor L \phi^{0}(x / L) / 2\right\rfloor \text { for even } x \\
& h_{x}^{0}:=2\left\lfloor\left(L \phi^{0}(x / L)-1\right) / 2\right\rfloor+1 \text { for odd } x
\end{aligned}
$$




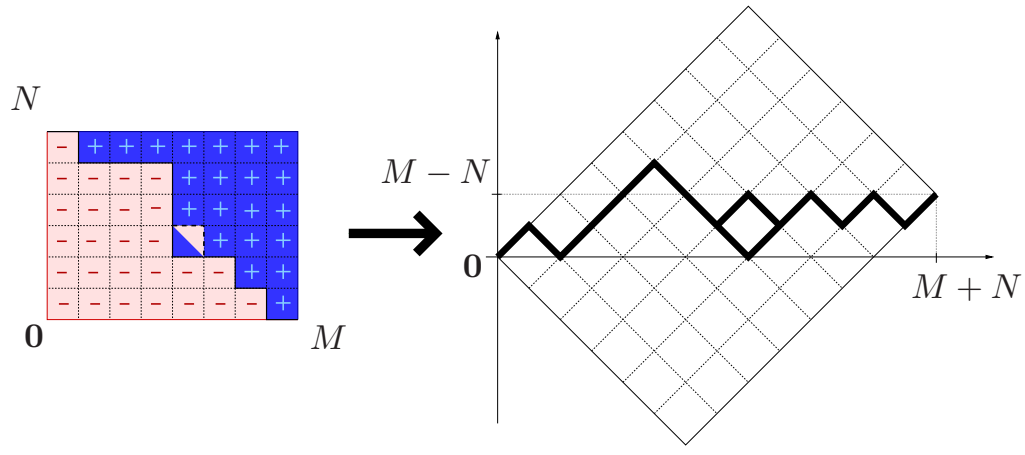

FiguRE 3. One-to-one correspondence between the dynamics in a rectangle with mixed boundary conditions and the corner-flip dynamics on paths. A possible spin update together with the equivalent corner-flip are represented

$\left(M_{N}\right.$ and $N_{L}$ are implicitly fixed by $L$ and $\left.\phi^{0}(1)\right)$. For all $T \geq 0$ and $\varepsilon>0$, w.h.p.

$$
\sup _{t \in[0, T], x \in[0,1]} \frac{1}{L}\left|h_{\lceil x L\rceil}\left(L^{2} t\right)-L \phi(x, t)\right| \leq \varepsilon
$$

where $\phi:[0,1] \times \mathbb{R}_{+} \rightarrow \mathbb{R}$ is the solution of the Cauchy problem

$$
\left\{\begin{array}{l}
\partial_{t} \phi(x, t)=\frac{1}{2} \partial_{x}^{2} \phi(x, t) \quad \forall t>0, \quad \forall x \in(0,1), \\
\phi(0, t)=0, \quad \phi(1, t)=\phi^{0}(1) \quad \forall t>0 \\
\phi(x, 0)=\phi^{0}(x) \quad \forall x \in(0,1) .
\end{array}\right.
$$

3.2. Local dynamics around the poles and a zero-range process. For the definition of the second auxiliary dynamics, we use the same notation as in the previous section, but no confusion should arise as the proofs will be given in two independent sections. The state space is

$$
\Omega_{L}:=\{h:\{-L, \ldots, L+1\} \mapsto \mathbb{Z}\} .
$$

For $h \in \Omega_{L}$ and $x \in\{-L+1, \ldots, L\}$ define $h^{+, x}$ (resp. $h^{-, x}$ ) as the configuration such that $h_{y}^{+, x}=h_{y}$ if $y \neq x$ and $h_{x}^{+, x}=h_{x}+1$ (resp. $h_{x}^{-, x}=h_{x}-1$ ). We consider the Markov chain $(h(t))_{t \geq 0}$ started from some $h^{0} \in \Omega_{L}$ and with generator $\mathcal{L}$ defined by

$$
\mathcal{L} f(h)=\frac{1}{2} \sum_{x=-L+1}^{L} c^{+, x}(h)\left(f\left(h^{+, x}\right)-f(h)\right)+c^{-, x}(h)\left(f\left(h^{-, x}\right)-f(h)\right)
$$

where

$$
\begin{aligned}
& c^{+, x}(h)=\mathbf{1}_{\left\{h_{x+1}>h_{x}\right\}}+\mathbf{1}_{\left\{h_{x-1}>h_{x}\right\}}, \\
& c^{-, x}(h)=\mathbf{1}_{\left\{h_{x+1}<h_{x}\right\}}+\mathbf{1}_{\left\{h_{x-1}<h_{x}\right\}} .
\end{aligned}
$$

Note that the values $h_{-L}$ and $h_{L+1}$ are fixed in time and should be considered as boundary conditions.

Remark 3.3. This dynamics corresponds to the motion of the interface for a modified Ising dynamics in a vertical strip of width $2 L$ with the following boundary condition: spins on the left (resp. right) boundary of the system are "+" if and only if their vertical coordinate is larger than $h_{-L}$ (resp. $\left.h_{L+1}\right)$. The dynamics is modified in the sense that updates are discarded if after the update the boundary between the "-" and "+" domain is not a simple (open) 
curve (see Figure 4). It is at times more convenient to identify $h \in \Omega_{L}$ with a càdlàg function $H:[-L-1 / 2, L+3 / 2] \mapsto \mathbb{Z}$ which equals identically $h_{n}$ on intervals $[n-1 / 2, n+1 / 2)$ for integer $n$.

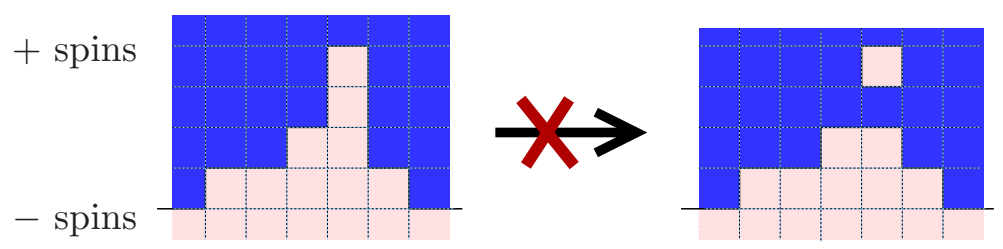

FiguRE 4. An example of spin update that splits the interface into two disconnected components. The interface dynamics presented in this section does not allow this kind of move.

Another way to interpret this dynamics [24] is to look at the gradients $\eta_{x}=h_{x+1}-h_{x}$ : one recognizes then a zero-range process with two type of particles (if $\eta_{x}=n>0$ we say there are $n$ particles of type $\mathrm{A}$ at $x$, if $\eta_{x}=-n<0$ we say there are $n$ type-B particles). Each particle performs a symmetric simple random walk with jump rate $1 /(2 n)$ (with $n$ the occupation number of the site where the particle sits) to either left or right and particles of different type annihilate instantaneously when they are at the same site. See Figure 5.

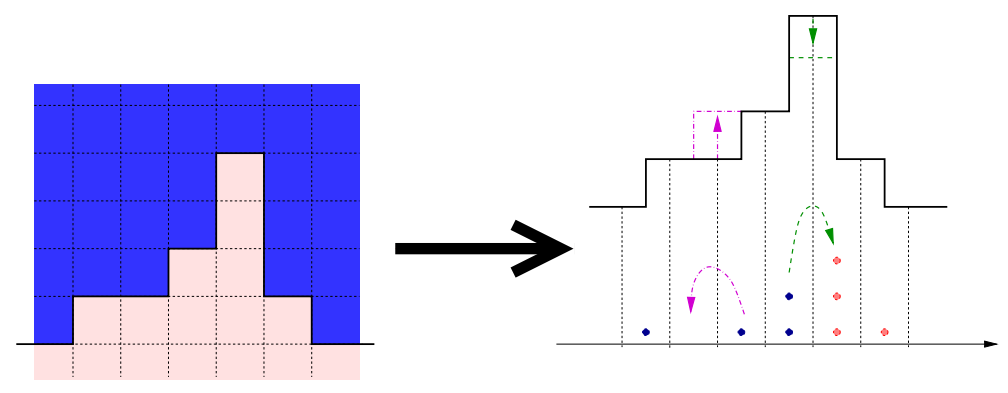

FiguRE 5. Correspondence between interface dynamics and zero-range process. Arrows represent possible motions for the interface and their representation in terms of particle moves. When an A particle jumps on a B particle (green arrow) both annihilate.

In [24, Appendix A], this dynamics was considered but in a periodized setup. A scaling limit result was given but the proof there is somewhat sketchy. Here we adapt the proof to the non-periodic case and write it in full details.

Consider $\phi^{0}:[-1,1] \mapsto \mathbb{R}$ a $C^{2}$ function with $\phi^{0}(1)=\phi^{0}(-1)=0$. We further assume that $\phi^{0}$ has a finite number of changes of monotonicity. Define $\Phi_{0}:\{-L, \ldots, L+1\} \mapsto \mathbb{R}$ as

$$
\Phi_{0}(x):=L \phi^{0}(x / L)
$$

and $h^{0}:\{-L, \ldots, L+1\} \mapsto \mathbb{Z}$ by

$$
h_{x}^{0}:=\left\lfloor\Phi_{0}(x)\right\rfloor .
$$


We define $\Phi:\{-L, \ldots, L+1\} \times \mathbb{R}_{+} \rightarrow \mathbb{R}$ as the solution of the following Cauchy problem:

$$
\begin{cases}\partial_{t} \Phi(x, t) & =\frac{1}{2}\left[\sigma\left(q_{x}(t)\right)-\sigma\left(q_{x-1}(t)\right)\right] \\ \Phi(L+1, t) & =\Phi(-L, t)=0 \\ \Phi(x, 0) & =\Phi_{0}(x)\end{cases}
$$

for every $t \geq 0$ and $x \in\{-L, \ldots, L+1\}$, where $\sigma(u)=u /(1+|u|)$ and

$$
q_{x}(t):=\Phi(x+1, t)-\Phi(x, t) .
$$

The result we state now is slightly weaker than Theorem 3.2 as it allows to control the profile $h$ only at a fixed time and not on a whole time interval.

Theorem 3.4. Given $\phi^{0}$ as above, consider $(h(t))_{t \geq 0}$ the dynamics described by (3.8) with initial condition $h^{0}$ as in (3.11). Then for any $t$, the following convergence holds in probability

$$
\lim _{L \rightarrow \infty} \max _{x \in\{-L, \ldots, L+1\}} \frac{1}{L}\left|h_{x}\left(L^{2} t\right)-\Phi\left(x, L^{2} t\right)\right|=0 .
$$

It is quite intuitive that one should have that $\frac{1}{L} \Phi\left(\lfloor L x\rfloor, L^{2} t\right) \rightarrow \phi(x, t)$ for any $x \in[-1,1]$, where $\phi:[-1,1] \times \mathbb{R}_{+} \rightarrow \mathbb{R}$ is the solution of

$$
\begin{cases}\partial_{t} \phi(x, t) & =\frac{1}{2} \frac{\partial_{x}^{2} \phi(x, t)}{\left(1+\left|\partial_{x} \phi(x, t)\right|\right)^{2}} \\ \phi(1, t) & =\phi(-1, t)=0 \\ \phi(x, 0) & =\phi^{0}(x)\end{cases}
$$

for $t \geq 0$ and $x \in(-1,1)$. The particular form of the non-linearity of this PDE makes the convergence question non-trivial, but fortunately Theorem 3.4 together with a comparison with the heat equation (cf. Section A.5) turns out to be sufficient for our purposes. Indeed, define $\bar{\phi}:[-1,1] \times \mathbb{R}_{+} \rightarrow \mathbb{R}$ to be the solution of

$$
\begin{cases}\partial_{t} \bar{\phi}(x, t) & =\frac{1}{2} \partial_{x}^{2} \bar{\phi}(x, t) \\ \bar{\phi}(1, t) & =\bar{\phi}(-1, t)=0 \\ \bar{\phi}(x, 0) & =\phi^{0}(x)\end{cases}
$$

Then

Corollary 3.5. Let $\phi^{0}$ be as above, and assume further that it is concave with $\left\|\partial_{x} \phi^{0}\right\|_{\infty} \leq \eta$. For every $t \geq 0$ and every $\varepsilon>0$ the following inequality holds w.h.p.

$$
\bar{\phi}(x / L, t)-\varepsilon \leq \frac{1}{L} h_{x}\left(L^{2} t\right) \leq \bar{\phi}\left(x / L,(1+\eta)^{-2} t\right)+\varepsilon \quad \text { for every } x \in\{-L, \ldots, L+1\} .
$$

Proof. The result follows by combining Theorem 3.4, Proposition A.9, and by taking limits of rescaled versions of $\Phi_{1}$ and $\Phi_{2}$ in (A.55) when $L$ tends to infinity (cf. Lemma 7.1).

3.3. About the scale-invariant shape. Now that we know how the interface should evolve locally (from Theorems 3.2 and 3.4) it is possible to explain why $\mathscr{D}$ should be scale invariant. By symmetries of the problem and the fact that motion is driven by curvature, the scale-invariant shape should be convex symmetric around the axes $\mathbb{R} \mathbf{e}_{\mathbf{1}}, \mathbb{R} \mathbf{e}_{\mathbf{2}}$. Therefore it is enough to consider the boundary of the intersection of $\mathscr{D}$ with the first quadrant.

From Theorem 3.2, if $f$ is a Lipschitz function and $\partial \mathscr{D}$ is the graph of $f$ in the coordinate system $\left(\mathbf{f}_{1}, \mathbf{f}_{2}\right)$, the initial drift in the $\mathbf{f}_{\mathbf{2}}$ direction is $(1 / 4) \partial_{x}^{2} f$, where the factor $1 / 4$ (instead of $1 / 2$ ) is due to the fact that in the correspondence between Ising dynamics and dynamics of nearest-neighboring paths, space has to be rescaled by $\sqrt{2}$, cf. Remark 3.1). One the other 
hand, the homothetic contraction of a shape $\mathcal{D}$ of initial velocity $\alpha$ gives an initial drift of the interface in the $\mathbf{f}_{2}$ direction

$$
\alpha\left(-f+x \partial_{x} f\right) .
$$

That leads to the partial differential equation

$$
\partial_{x}^{2} f=4 \alpha\left(-f+x \partial_{x} f\right) .
$$

Next we impose the correct boundary conditions on $f$ :

- We fix the scaling by imposing that the point $(1,0)$ (and therefore also $(0,1),(-1,0),(0,-1)$ ) belongs to $\partial \mathscr{D}$. This gives

$$
f( \pm 1 / \sqrt{2})=1 / \sqrt{2} .
$$

- To guarantee that the curvature of $\partial \mathscr{D}$ is well defined at the point $(0,1)$ we have to impose

$$
\partial_{x} f(-1 / \sqrt{2})=-\partial_{x} f(1 / \sqrt{2})=1 \text {. }
$$

We finally notice that

Lemma 3.6. The function $f_{0}$ defined in (2.12) is the unique solution of the Cauchy problem (3.19)-(3.20)-(3.21) for $x \in(-1 / \sqrt{2},+1 / \sqrt{2})$. For other values of $\alpha$ the above problem has no solution.

Proof. Uniqueness of the solution is standard from theory of ordinary differential equation. The rest is just a matter of checking.

3.4. Organization of the paper. Instead of proving directly Theorem 2.2 and then deducing Theorem 2.3 as a corollary, we decided for pedagogical reasons to give first the proof in the case of the scale-invariant droplet and then to point out what needs to be modified in the more general case of a convex droplet. The reason is that, this way, we can easily separate the question of comparing the stochastic evolution with the deterministic one (which works more or less the same in the two cases but is simpler for the invariant droplet, due to its symmetries) from the analytic, PDE-type issues which appear only in the general case.

The paper is therefore organized as follows:

- in Section 4, we show that to prove Theorem 2.3 it is sufficient to have a good control on the continuity of the interface motion (Proposition 4.2) and a result on the evolution after an "infinitesimal time" $\varepsilon L^{2}$ (Proposition 4.1). Such crucial results are proven in Sections 4.3 and 4.4 ;

- in Section 5 we first prove Theorem 2.1 on the existence of a solution to (2.7), and then we prove Theorem 2.2 via a suitable generalization of Propositions 4.2 and 4.1;

- finally, the hydrodynamic limit results of Theorems 3.2 and 3.4 are proven in detail in Section 7 and the Appendix A respectively.

\section{Proof of Theorem 2.3: evolution of the SCAle-Invariant Droplet}

4.1. Reducing to an "infinitesimal" time interval. We decompose the proof of Theorem 2.3 into two propositions. The first (and the main one) says that after a time $\varepsilon L^{2}$ the droplet looks very much the same but contracted by a factor $(1-\alpha \varepsilon+o(\varepsilon))$. 
Proposition 4.1. For all $\delta>0$ there exists $\varepsilon_{0}(\delta)>0$ such that for all $0<\varepsilon<\varepsilon_{0}(\delta)$, w.h.p.,

$$
\mathcal{A}_{L}\left(L^{2} \varepsilon\right) \subset(1-\varepsilon(\alpha-\delta)) L \mathscr{D},
$$

and

$$
\mathcal{A}_{L}\left(L^{2} \varepsilon\right) \supset(1-\varepsilon(\alpha+\delta)) L \mathscr{D} .
$$

The second proposition controls continuity in time of the rescaled motion:

Proposition 4.2. For every $\delta>0$, w.h.p.,

$$
\mathcal{A}_{L}\left(L^{2} t\right) \subset(1+\delta) L \mathscr{D} \text { for every } t \geq 0 .
$$

Moreover, for every $\delta>0$ there exists $\varepsilon>0$ such that w.h.p

$$
\mathcal{A}_{L}\left(L^{2} t\right) \supset(1-\delta) L \mathscr{D} \text { for every } t \in[0, \varepsilon] .
$$

Proof of Theorem 2.3 assuming Propositions 4.1 and 4.2. Given $\eta$ fix $\delta$ small enough and $\varepsilon<$ $\varepsilon_{0}(\delta)$. Then using (4.1) one gets that w.h.p.

$$
\mathcal{A}_{L}\left(L^{2} \varepsilon\right) \subset(1-(\alpha-\delta) \varepsilon) L \mathscr{D} .
$$

Let $\left(\mathcal{A}_{L}^{(1)}\left(L^{2} t\right)\right)_{t \geq 0}$ denote the evolution of the set of " -" spins for the dynamics started from initial condition "-" on $(1-\varepsilon(\alpha-\delta)) L \mathscr{D}$ and "+" elsewhere. Then using the Markov property and monotonicity of the dynamics, one can couple the dynamics $\left(\mathcal{A}_{L}\left(L^{2}(\varepsilon+t)\right)\right)_{t \geq 0}$ and $\left(\mathcal{A}_{L}^{(1)}\left(L^{2} t\right)\right)_{t \geq 0}$ such that on the event $(4.5)$

$$
\mathcal{A}_{L}\left(L^{2}(\varepsilon+t)\right) \subset \mathcal{A}_{L}^{(1)}\left(L^{2} t\right), \quad \text { for every } t \geq 0 .
$$

Therefore, after conditioning to the event in (4.5) and using (4.1) for $(1-(\alpha-\delta) \varepsilon) L$ instead of $L$, one gets that w.h.p.:

$$
\mathcal{A}_{L}\left(L^{2} \varepsilon\left(1+(1-(\alpha-\delta) \varepsilon)^{2}\right)\right) \subset \mathcal{A}_{L}^{(1)}\left(L^{2}(1-(\alpha-\delta) \varepsilon)^{2} \varepsilon\right) \subset(1-(\alpha-\delta) \varepsilon)^{2} L \mathscr{D} .
$$

Using this argument repeatedly one gets that, w.h.p., for all $k \in\left[1, \varepsilon^{-3 / 2}\right]$ (here and in the sequel we assume $\varepsilon^{-3 / 2}$ to be in $\mathbb{N}$ )

$$
\mathcal{A}_{L}\left(L^{2} t_{k}\right) \subset(1-(\alpha-\delta) \varepsilon)^{k} L \mathscr{D}
$$

where $t_{k}$ is defined by

$$
t_{k}:=\varepsilon \sum_{i=0}^{k-1}(1-(\alpha-\delta) \varepsilon)^{2 i}=\varepsilon \frac{1-(1-(\alpha-\delta) \varepsilon))^{2 k}}{1-(1-(\alpha-\delta) \varepsilon)^{2}} .
$$

One remarks that for all values of $k \in\left[1, \varepsilon^{-3 / 2}\right]$,

$$
\begin{aligned}
(1-(\alpha-\delta) \varepsilon)^{k} & =\sqrt{1-\frac{t_{k}\left(1-(1-(\alpha-\delta) \varepsilon)^{2}\right)}{\varepsilon}} \\
& =\sqrt{1-2(\alpha-\delta) t_{k}+t_{k} O(\varepsilon)} .
\end{aligned}
$$

As $\left(t_{k}\right)_{k \geqslant 0}$ is bounded above, there exists $C>0$ such that for every $k \in\left[1, \varepsilon^{-3 / 2}\right]$

$$
\mathcal{A}_{L}\left(L^{2} t_{k}\right) \subset\left(\sqrt{1-2(\alpha-\delta) t_{k}+C \varepsilon}\right) L \mathscr{D} \subset\left(\sqrt{1-2 \alpha t_{k}}+\eta / 2\right) L \mathscr{D}
$$

w.h.p. where the second inclusion holds provided that $\varepsilon$ and $\delta$ are small enough. Combining (4.11), Proposition 4.2 and stochastic coupling, one gets w.h.p. that, for every $k \in\left[0, \varepsilon^{-3 / 2}\right]$ and $t \in\left(t_{k}, t_{k+1}\right)$,

$$
\mathcal{A}_{L}\left(L^{2} t\right) \subset\left(\sqrt{1-2 \alpha t_{k}}+(3 \eta / 4)\right) L \mathscr{D} \subset(\sqrt{1-2 \alpha t}+\eta) L \mathscr{D}
$$


and that, w.h.p., for every $t \geq t_{\varepsilon^{-3 / 2}}$

$$
\mathcal{A}_{L}\left(L^{2} t\right) \subset\left(\sqrt{1-2 \alpha t_{\varepsilon^{-3 / 2}}}+(3 \eta / 4)\right) L \mathscr{D} \subset \eta L \mathscr{D} .
$$

This ends the proof of the upper inclusion in (2.17) (note that $t_{\varepsilon^{-3 / 2}}$ approaches $1 /(2 \alpha)$ for $\varepsilon, \delta$ small). Moreover, (4.13) and stochastic domination implies that, for some constant $C$, w.h.p.

$$
\tau_{+} \leq \frac{L^{2}}{2 \alpha}\left(1+C \eta^{2}\right)
$$

Indeed, it is known from [9] that a droplet of minus spins of linear size $\eta L$ disappears within a time $\tau_{+}$which w.h.p. is upper bounded by $C \eta^{2} L^{2}$.

The lower inclusion in (2.17) and the lower bound on $\tau_{+}$are proved in an analogous way using (4.2) instead of (4.1) and (4.4) instead of (4.3). Note that using (4.4) we have to take care to choose $\varepsilon$ small enough but it is possible as $t_{k}-t_{k-1}$ is a non-increasing function of $k$ and $\varepsilon$.

4.2. Strategy of the proof of Proposition 4.1. Our aim is to use Theorem 3.2 to control the motion of the interface away from the "poles" and Theorem 3.4 (or more precisely Corollary 3.5) to control the motion of the interface close to the "poles". It is therefore crucial to compare the local SSEP or the zero-range dynamics introduced in Sections 3.1 and 3.2 to the true evolution of the boundary between "+" and "-" spins.

As we have already discussed at the beginning of Section 3, however, there exists no exact mapping between the evolution of the height function associated to the two particle processes and the evolution of the +/- boundary, since the original "-" droplet can break into more droplets and, strictly speaking, the interface cannot be described, even locally, as a height function. The way out is that, thanks to monotonicity arguments and to the a priori "continuity" information provided by Proposition 4.2, we can remove certain updates of the Markov Chain, e.g. freeze certain spins to their initial value. This way, we can show that locally the interface can be stochastically compared to the height function associated to the SSEP (or to the zero-range process close to the poles). Of course, the detail of the "update removal procedure" is quite different according to whether we want to prove an upper or a lower bound on the "- domain". For instance, if we want an upper bound we are allowed to freeze "-" spins or to change some "+" into "-" spins in the initial condition (this is fine thanks to monotonicity) and at the same time we can freeze the spins outside $(1+\delta) L \mathscr{D}$ to "+" (this is not allowed directly by monotonicity, but (4.3) guarantees that such spins stay "+" for all times anyway, w.h.p.). If the "update removal procedure" is performed suitably, the effect is that the various portions of the $+/$ - interface (away from and close to the poles) then become independent and evolve exactly like the height functions of the SSEP/zero-range process.

The approach outlined here will be also used in Section 6 in the case with general convex initial condition (the generalization of Proposition 4.1 is Proposition 6.2).

4.3. Upper Bound: proof of (4.1) and (4.3). The inclusion (4.1) can be rewritten in the following manner, which is more convenient for the proof: for any positive $\delta$, for all $\varepsilon$ small enough, w.h.p.

$$
\sigma_{x}\left(\varepsilon L^{2}\right)=+ \text { for every } x \in[(1-\varepsilon(\alpha-\delta)) L \mathscr{D}]^{c} .
$$

Given $\delta$, we fix a value of $\xi$ which is small enough (depending on $\delta$ in a way that is specified in Section 4.3.2) and set (cf. Figure 6)

$$
\begin{aligned}
& M(\varepsilon, \xi):=\left\{(x, y) \in \mathbb{R}^{2}, x \geqslant \xi \text { and } y \geqslant \xi\right\} \backslash[(1-\varepsilon(\alpha-\delta)) \mathscr{D}] \\
& N(\varepsilon, \xi):=\left\{(x, y) \in \mathbb{R}^{2}, y \geqslant 0 \text { and }-\xi \leqslant x \leqslant \xi\right\} \backslash[(1-\varepsilon(\alpha-\delta)) \mathscr{D}] .
\end{aligned}
$$


Remark that for any $\varepsilon>0, M, N$ and their successive images by rotation of angle $\pi / 2, \pi, 3 \pi / 2$ form an 8-piece cover of the complementary set $[(1-\varepsilon(\alpha-\delta)) \mathscr{D}]^{c}$.

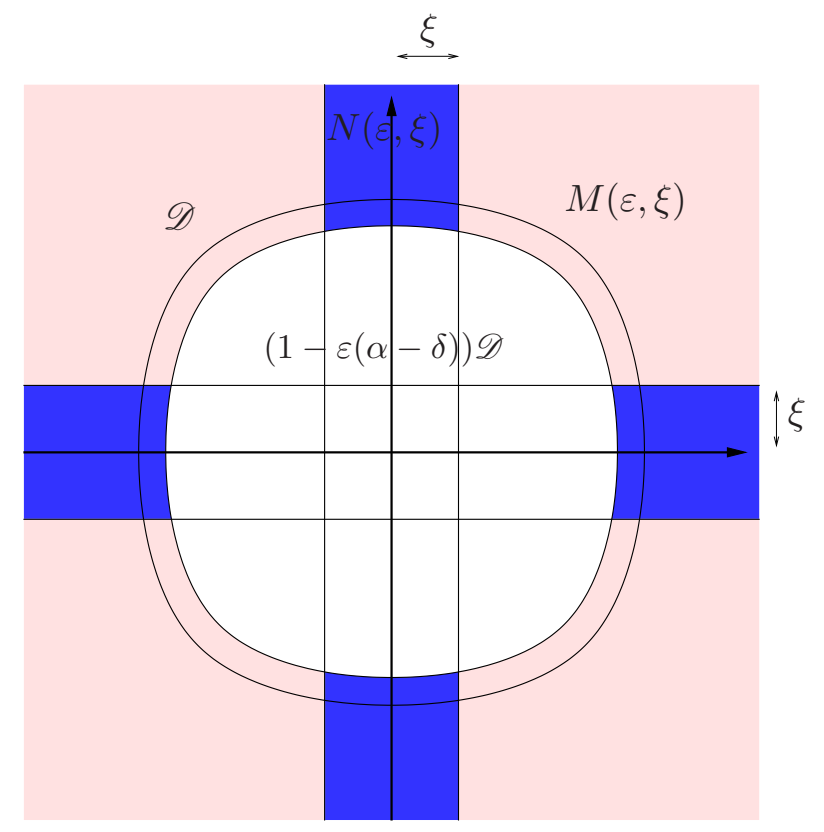

Figure 6. The light-colored (resp. dark-colored) zones correspond $M(\varepsilon, \xi)$ (resp. $N(\varepsilon, \xi))$ and its rotations. Together, they form a partition of the complement of $(1-\varepsilon(\alpha-\delta)) \mathscr{D}$ (white central region).

As the dynamics and the initial shape are invariant under these same rotations, (4.15) is proved if we can show that for $\varepsilon$ small enough, w.h.p.

$$
\begin{aligned}
& \sigma_{x}\left(\varepsilon L^{2}\right)=+ \text { for every } x \in \operatorname{LM}(\varepsilon, \xi) \\
& \sigma_{x}\left(\varepsilon L^{2}\right)=+ \text { for every } x \in \operatorname{LN}(\varepsilon, \xi) .
\end{aligned}
$$

The above new formulation of (4.1) is very convenient as it allows to consider separately the dynamics close to the poles and away from them.

4.3.1. Proof of (4.17). For any $L>0$, we consider the dynamics which has initial condition with "-" spins in $L \mathscr{D}$ and "+" otherwise, and the same generator as the original dynamics except that spins on the sites in $V_{1}:=\left\{ \pm \frac{1}{2}\right\} \times\left\{-L+\frac{1}{2}, \cdots, L-\frac{1}{2}\right\}$ and on $V_{2}:=\left\{-L+\frac{1}{2}, \cdots, L-\frac{1}{2}\right\} \times\left\{ \pm \frac{1}{2}\right\}$ are "frozen to -". (The construction of the dynamics is the same as in Section 2.3, except that there is no update for these sites). We denote $\left(\sigma_{L}^{(1)}(t)\right)_{t \geq 0}$ the evolution of this dynamics and define

$$
\mathcal{A}_{L}^{(1)}(t):=\bigcup_{\left\{x: \sigma_{x}^{(1)}(t)=-1\right\}} \mathcal{C}_{x} .
$$

The graphical construction of Section 2.3 gives a natural coupling of $\sigma$ and $\sigma^{(1)}$ :

$$
\mathcal{A}_{L}(t) \subset \mathcal{A}_{L}^{(1)}(t), \text { for every } t \geqslant 0 .
$$

The advantage of the "freezing procedure" is that then the evolution in the four quadrants of $\left(\mathbb{Z}^{*}\right)^{2}$ becomes independent. The reason is that the spins on sites $\left(\left\{ \pm \frac{1}{2}\right\} \times \mathbb{Z}^{*}\right) \backslash V_{1}$ and $\left(\mathbb{Z}^{*} \times\left\{ \pm \frac{1}{2}\right\}\right) \backslash V_{2}$ are "+" for all times, since they always have at least three "+" neighbors. 
The set $\mathcal{A}_{L}^{(1)}(t) \cap \mathbb{R}_{+}^{2}$ is a Young diagram (i.e. a collection of vertical columns of width 1 and non-negative integer heights, with heights non-increasing from left to right) for all $t \geq 0$ and we can thus consider $\partial \mathcal{A}_{L}^{(1)}(t) \cap \mathbb{R}_{+}^{2}$ as the graph of a (random) piecewise linear function in the coordinate system $\left(\mathbf{f}_{1}, \mathbf{f}_{2}\right)$, that we denote by $F_{L}(\cdot, t)$. Equation $(4.17)$ is thus proved (for any choice of $\xi$ ) if one proves that for any $\nu<2^{-1 / 2}$, for any $\varepsilon$ small enough, w.h.p.

$$
F_{L}\left(x, \varepsilon L^{2}\right) \leq L f((\alpha-\delta) \varepsilon, x / L) \text { for every } x \in(-\nu L, \nu L),
$$

where $f(\cdot, t)$ is the function whose graph in the coordinate system $\left(\mathbf{f}_{1}, \mathbf{f}_{2}\right)$ is given by the intersection of the boundary of $(1-t) \mathscr{D}$ with the half-plane $\left\{(x, y) \in \mathbb{R}^{2},(x, y) \cdot \mathbf{f}_{2} \geqslant 0\right\}$ (the domain of definition of $f(t, \cdot)$ depends on $t$ but includes $\left[-2^{-1 / 2}, 2^{-1 / 2}\right]$ for $t$ small enough). By definition of $\mathscr{D}$, one has $f(\cdot, 0)=f_{0}(\cdot)$ (recall the definition of $f_{0}$ in $(2.12)$ ).

In practice, to prove (4.17) one has to prove (4.21) with $\nu$ such that $1 / \sqrt{2}-\nu=\xi / \sqrt{2}+o(\xi)$ for $\xi$ small. The reason is that the point of $\partial \mathscr{D}$ with horizontal coordinate $\xi$ and positive vertical coordinate (in the coordinate system $\left(\mathbf{e}_{\mathbf{1}}, \mathbf{e}_{\mathbf{2}}\right)$ ) has horizontal coordinate $-(1-\xi) / \sqrt{2}+o(\xi)$ in the $\left(\mathbf{f}_{1}, \mathbf{f}_{2}\right)$ coordinate system.

As explained in Remark 3.1 and on Figure 3, the function $F_{L}(\cdot, t)$, up to space rescaling (by a factor $\sqrt{2}$ ) undergoes the corner flip-dynamics of Theorem 3.2. Thus the scaling limit of $F_{L}$ satisfies the heat-equation or more precisely we have the following convergence in probability for every fixed $T>0$ :

$$
\lim _{L \rightarrow \infty} \sup _{x \in\left[-\frac{1}{\sqrt{2}}, \frac{1}{\sqrt{2}}\right]} \sup _{t \leqslant T}\left|\frac{1}{L} F_{L}\left(x L, t L^{2}\right)-g(x, t)\right|=0
$$

where $g$ is the solution for $t \geq 0$ and $x \in(-1 / \sqrt{2}, 1 / \sqrt{2})$ of

$$
\left\{\begin{array}{l}
\partial_{t} g(x, t)=\frac{1}{4} \partial_{x}^{2} g(x, t) \\
g(\cdot, 0)=f_{0}(\cdot) \\
g\left(-\frac{1}{\sqrt{2}}, t\right)=g\left(\frac{1}{\sqrt{2}}, t\right)=\frac{1}{\sqrt{2}} .
\end{array}\right.
$$

Note that the above result plus equation (4.20), plus the fact that $g$ is decreasing in $t$ (since it stays concave through time) gives (4.3) of Proposition 4.2 for every $t \leq T<\infty$. Moreover, according to [9, Theorem 1.3], the disappearance time $\tau_{+}$of the minus-droplet is $O\left(L^{2}\right)$ with high probability, so that (4.3) also holds for $t>T$ provided that $T$ was chosen large enough. As a byproduct, we have proven (4.3).

Concerning (4.17), in order to prove (4.21) we are reduced to show that for every $\nu \in(0,1 / \sqrt{2})$ and every $x \in(-\nu, \nu)$,

$$
g(x, \varepsilon)<f(x,(\alpha-\delta) \varepsilon) .
$$

This is a consequence of the way $f_{0}$ was determined (see (3.19) and discussion in Section 3.3). First we notice that the time derivative of $g$ is uniformly continuous away from the boundary points $\pm 1 / \sqrt{2}$ :

Lemma 4.3. For any $0<\nu<\frac{1}{\sqrt{2}}$,

$$
\lim _{t \rightarrow 0} \sup \left\{\left|\partial_{t} g(x, s)-\partial_{t} g(x, 0)\right|, s \in[0, t] \text { and } x \in[-\nu, \nu]\right\}=0 .
$$

Proof of Lemma 4.3. This is well known but we sketch a probabilistic proof for the sake of completeness. Let $\left(B_{t}\right)_{t} \geqslant 0$ denote a standard Brownian motion starting at $x \in\left[-2^{-1 / 2}, 2^{-1 / 2}\right]$ (with the associated expectation denoted by $E_{x}$ ) and let $T$ denote the hitting time of $\{ \pm 1 / \sqrt{2}\}$. One has

$$
\partial_{x}^{2} g(x, t)=E_{x}\left[\partial_{x}^{2} f_{0}\left(B_{t}\right) \mathbf{1}_{\{t<T\}}\right] .
$$


We can thus rewrite (4.25) as

$$
\lim _{t \rightarrow 0} \sup \left\{\left|E_{x}\left(\partial_{x}^{2} f_{0}\left(B_{s}\right)-\partial_{x}^{2} f_{0}(x)\right)\right|, s \in[0, t] \text { and } x \in[-\nu, \nu]\right\}=0
$$

and we can conclude using the uniform continuity of $\partial_{x}^{2} f_{0}$ on $\left[-\frac{1}{\sqrt{2}}, \frac{1}{\sqrt{2}}\right]$ and well-known continuity properties of the Brownian motion. Remark that (4.25) would not hold with $\nu=1 / \sqrt{2}$ because of boundary effects: for $t>0$ one has that $\partial_{x}^{2} g(x, t)$ approaches zero as $x$ approaches $\pm 1 / \sqrt{2}$, since $P_{x}(T>t) \rightarrow 0$ when $x \rightarrow \pm 1 / \sqrt{2}$.

Therefore, for every $\eta>0$ arbitrarily small we have for all $x$ in $(-\nu, \nu)$, if $\varepsilon$ is small enough,

$$
g(x, \varepsilon)<f_{0}(x)+\varepsilon\left(\frac{1}{4} \partial_{x}^{2} f_{0}(x)+\eta\right) .
$$

We are left with proving that for $\varepsilon$ small enough and $x$ in $(-\nu, \nu)$

$$
f_{0}(x)+\varepsilon\left(\frac{1}{4} \partial_{x}^{2} f_{0}(x)+\eta\right)<f(x,(\alpha-\delta) \varepsilon) .
$$

From the definition of $f(\cdot, t)$ as graph in $\left(\mathbf{f}_{1}, \mathbf{f}_{2}\right)$ of the boundary of $(1-t) \mathscr{D}$, we get that if $\varepsilon$ is small enough, uniformly for all $x \in(-\nu, \nu)$,

$$
\begin{gathered}
f(x,(\alpha-\delta) \varepsilon)=[1-(\alpha-\delta) \varepsilon] f\left(\frac{x}{1-(\alpha-\delta) \varepsilon}, 0\right) \\
=f_{0}(x)+(\alpha-\delta) \varepsilon\left(x \partial_{x} f_{0}(x)-f_{0}(x)\right)+O\left(\varepsilon^{2}\right) .
\end{gathered}
$$

Now recall that $f_{0}$ satisfies equation (3.19), so we are reduced to check that for all $x \in(-\nu, \nu)$,

$$
\eta+\delta\left(x \partial_{x} f_{0}(x)-f_{0}(x)\right)=\eta+\frac{\delta}{4 \alpha} \partial_{x}^{2} f_{0}<0
$$

which holds provided $\eta=\eta(\delta)$ is small, since $\partial_{x}^{2} f_{0}(\cdot)$ is negative and uniformly bounded away from zero. Equation (4.17) is proven.

4.3.2. Proof of (4.18). The method is similar to the one we used for (4.17), the main difference being that, via a chain of monotonicity arguments, we analyze the evolution of the portion of interface near the "poles" by comparing it to the interface dynamics of Section 3.2 (which coincides with the height function of the zero-range process with two types of particles) instead of the "corner-flip dynamics".

Denote by $h(\cdot, t)$ the function whose graph in the coordinates system $\left(\mathbf{e}_{1}, \mathbf{e}_{2}\right)$ is given by the intersection of $(1-t) \partial \mathscr{D}$ with the upper half-plane $\mathbb{R} \times \mathbb{R}_{+}$, and $h_{0}(\cdot)=h(\cdot, 0)$. Note that $h_{0}$ is $C^{\infty}$ on $(-1,0)$ and on $(0,+1)$ by the definition of $\mathscr{D}$. The boundary condition (3.21) ensures continuity of the first derivative of $h_{0}$ at zero $\left(\partial_{x} h_{0}(0)=0\right)$; the reader can check that $h_{0}$ has also continuous second derivative and that

$$
\partial_{x}^{2} h_{0}(0)=\frac{1}{2 \sqrt{2}} \partial_{x}^{2} f_{0}(-1 / \sqrt{2})=-2 \alpha
$$

but that the third derivative exhibits a discontinuity in 0 .

Recall that $\xi$ is the positive constant appearing in (4.16). We set $\bar{h}:[-4 \xi, 4 \xi] \mapsto \mathbb{R}$ to be the function defined by the following conditions: $\bar{h} \equiv h_{0}$ on $[-2 \xi, 2 \xi], \bar{h}$ is affine on $[-4 \xi,-2 \xi]$ and on $[2 \xi, 4 \xi]$ and the derivative $\partial_{x} \bar{h}(\cdot)$ is continuous on $(-4 \xi, 4 \xi)$. Since $h_{0}(\cdot)$ is strictly convex, we have $h_{0}(x) \leq \bar{h}(x)$ with strict inequality outside $[-2 \xi, 2 \xi]$. Define also the following subsets of $\mathbb{R}^{2}$ (cf. Figure 7 ):

$$
J^{1}:=[4 \xi, \infty) \times[\bar{h}(4 \xi), \infty) \quad J^{2}:=(-\infty,-4 \xi] \times[\bar{h}(4 \xi), \infty) .
$$


To avoid notational complications with integer parts, we assume that $L \bar{h}(4 \xi)$ and $4 L \xi$ belong to $\mathbb{Z}^{*}$.

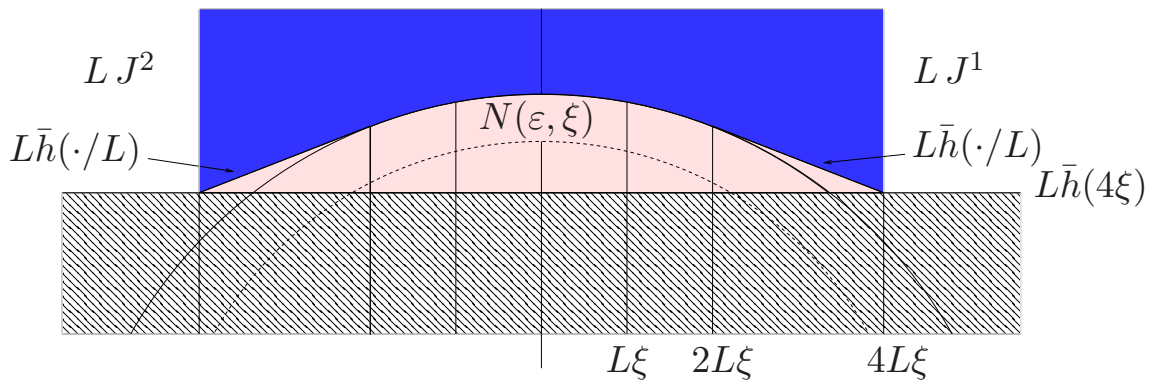

FiguRE 7. In the set $L\left(J^{1} \cup J^{2}\right)$ spins are frozen to "+" while in the dashed region they are is frozen to "-". The initial condition is "+" in the dark-colored region and "-" in the light-colored one. The boundary separating dark/light region is determined by the function $\bar{h}(\cdot)$.

First of all, observe that, thanks to (4.1), we can freeze the spins in $L\left(J^{1} \cup J^{2}\right)$ to their initial value "+" and, w.h.p., the dynamics will be identical for all times to the original one.

Next, we employ a chain of monotonicities, based on the graphical construction of Section 2.3. Since we are after an upper bound on the set of minus spins, we can freeze to "-" all spins whose vertical coordinate is below $L \bar{h}(4 \xi)$. Therefore, we have just a dynamics in the set

$$
Y:=[-4 L \xi+1,4 L \xi-1] \times[L \bar{h}(4 \xi), \infty) .
$$

In principle, its initial condition is such that the spin at site $\left(x_{1}, x_{2}\right) \in Y$ is "-" if and only if $x_{2} \in\left[L \bar{h}(4 \xi), L h_{0}\left(x_{1} / L\right)\right]$. The problem is however that the function $x \mapsto \max \left(\bar{h}(4 \xi), h_{0}(x)\right)$ is not concave, which prevents to apply directly Corollary 3.5 later. By monotonicity, we can modify such initial condition by adding extra " -" spins: we therefore stipulate that at time $t=0$ the spin at site $\left(x_{1}, x_{2}\right)$ is " -" if and only if $x_{2} \in\left[L \bar{h}(4 \xi), L \bar{h}\left(x_{1} / L\right)\right]$. Recall that $\bar{h}(x) \geq h_{0}(x)$, so monotonicity goes in the correct direction. With some abuse of notation, we still call $(\sigma(t))_{t \geq 0}$ the dynamics thus modified and $\mathcal{A}_{L}(t)$ the set of minus spins. We need a final step in order to map the evolution into the zero-range process. Note that, at time $t=0$, the boundary of $\mathcal{A}_{L}(t=0)$, intersected with the strip $[-4 L \xi+1 / 2,4 L \xi-1 / 2] \times \mathbb{R}$, can be identified with the graph of a càdlàg function

$$
H_{L}(\cdot, 0):[-4 L \xi+1 / 2,4 L \xi-1 / 2] \mapsto[L \bar{h}(4 \xi)-1 / 2, \infty) \cap \mathbb{Z},
$$

which is constant on intervals $[n, n+1)$ with $n \in \mathbb{Z}$ and takes boundary values $L \bar{h}(4 \xi)-1 / 2$ at the two endpoints $\left(H_{L}(x, 0)\right.$ is just a discretized version of $\left.L \bar{h}(x / L)\right)$. However, for time $t>0$ it is not true in general that the boundary of $\mathcal{A}_{L}(t)$ is still the graph of a function, simply because the set $\mathcal{A}_{L}(t)$ can be non-connected (see Figure 4 ). Let $\left(\sigma^{(2)}(t)\right)_{t \geq 0}$ be the dynamics obtained by erasing all the updates that would make $\mathcal{A}_{L}(t)$ non-connected. It is easy to realize that, since $H_{L}(\cdot, 0)$ has a single change of monotonicity (from non-decreasing to non-increasing, recall that $\bar{h}(\cdot)$ is concave) such erased updates can only correspond to a "-" spin turning into a "+" spin (see again Figure 4). Therefore, the set of minus spins of the dynamics $\left(\sigma^{(2)}(t)\right)_{t \geq 0}$ dominates stochastically $\mathcal{A}_{L}(t)$ : more precisely, we have shown that the coupling given by the graphical 
construction implies that, w.h.p. and for all $t \geq 0$,

$$
\mathcal{A}_{L}(t) \subset \mathcal{A}_{L}^{(2)}(t):=\bigcup_{\left\{x: \sigma_{x}^{(2)}(t)=-\right\}} \mathcal{C}_{x} .
$$

We let

$$
H_{L}(\cdot, t):[-4 L \xi+1 / 2,4 L \xi-1 / 2] \mapsto[L \bar{h}(4 \xi)-1 / 2, \infty) \cap \mathbb{Z},
$$

denote the piecewise constant (random) function whose graph in the usual coordinates system $\left(\mathbf{e}_{1}, \mathbf{e}_{2}\right)$ is the intersection between $\partial \mathcal{A}_{L}^{(2)}(t)$ and the strip $[-4 L \xi+1 / 2,4 L \xi-1 / 2] \times \mathbb{R}$. Note that $H_{L}(-4 L \xi+1 / 2, t)=H_{L}(4 L \xi-1 / 2, t)=L \bar{h}(4 \xi)-1 / 2$.

Equation (4.18) is proved if one shows that for any $\varepsilon$ small enough, w.h.p.

$$
\frac{1}{L} H_{L}\left(x, \varepsilon L^{2}\right) \leq h(x / L,(\alpha-\delta) \varepsilon) \text { for every } x \in(-\xi L, \xi L) .
$$

It is clear from Remark 3.3 that the function $H_{L}(\cdot, \cdot)$ follows the dynamics described in Section 3.2 , with generator $(3.3)$ (here we identify the function $H_{L}(\cdot, t)$ with an element of $\Omega_{4 \xi L-1 / 2}$, see (3.7)). According to Corollary 3.5 one has for arbitrarily small $\eta>0$, w.h.p, for all $x \in(-\xi L, \xi L)$

$$
\frac{1}{L} H_{L}\left(x, \varepsilon L^{2}\right) \leq \bar{\phi}\left(x / L,\left(1+\left\|\partial_{x} \bar{h}\right\|_{\infty}\right)^{-2} \varepsilon\right)+\eta
$$

where $\left\|\partial_{x} \bar{h}\right\|_{\infty}=\sup _{[-4 \xi, 4 \xi]}\left|\partial_{x} \bar{h}(x)\right|$ and $\bar{\phi}\left(x, L^{2} t\right)$ is the solution of

$$
\begin{cases}\partial_{t} \bar{\phi}(x, t) & =\frac{1}{2} \partial_{x}^{2} \bar{\phi}(x, t) \\ \bar{\phi}(-4 \xi, t) & =\bar{\phi}(4 \xi, t)=\bar{h}(4 \xi) \\ \bar{\phi}(x, 0) & =\bar{h}(x) \quad \text { for every } x \in[-4 \xi, 4 \xi] .\end{cases}
$$

The equation (4.36) is thus proved if one has

$$
\bar{\phi}\left(x,\left(1+\left\|\partial_{x} \bar{h}\right\|_{\infty}\right)^{-2} \varepsilon\right)<h(x,(\alpha-\delta) \varepsilon) \text { for every } x \in[-\xi, \xi] .
$$

Note that by Lemma 4.3 (which is applicable because the second derivative of $\bar{h}(\cdot)=h_{0}(\cdot)$ is uniformly continuous in $(-2 \xi, 2 \xi))$ one has, uniformly on $[-\xi, \xi]$

$$
\begin{aligned}
\bar{\phi}\left(x,\left(1+\left\|\partial_{x} \bar{h}\right\|_{\infty}\right)^{-2} \varepsilon\right)=\bar{\phi}(x, 0) & +\frac{\varepsilon}{2}\left(1+\left\|\partial_{x} \bar{h}\right\|_{\infty}\right)^{-2} \partial_{x}^{2} \bar{\phi}(x, 0)+o(\varepsilon) \\
& =h_{0}(x)+\frac{1}{2}\left(1+\left\|\partial_{x} \bar{h}\right\|_{\infty}\right)^{-2}\left(\partial_{x}^{2} h_{0}(0)+r(x)\right) \varepsilon+o(\varepsilon)
\end{aligned}
$$

where $r(x)$ tends to 0 for $x \rightarrow 0$. Finally, using (4.33), if $\xi$ is chosen small enough so that both $r(x)$ and $\left\|\partial_{x} \bar{h}\right\|_{\infty}$ are sufficiently smaller than $\delta$,

$$
\bar{\phi}\left(x,\left(1+\left\|\partial_{x} \bar{h}\right\|_{\infty}\right)^{-2} \varepsilon\right) \leq h_{0}(x)-(\alpha-\delta / 4) \varepsilon .
$$

On the other hand one has

$$
h(x,(\alpha-\delta) \varepsilon) \geq h_{0}(x)-(\alpha-\delta / 2) \varepsilon,
$$

which ends the proof of (4.18).

4.4. Lower bound: proof of (4.4) and (4.2). The proofs follow the same ideas as those of Section 4.3: we need to control the dynamics for different portions of the interface separately (around the poles and away from them) using the scaling limit results provided by Theorems 3.4 and 3.2 . 
4.4.1. Proof of (4.4). Equation (4.4) is absolutely crucial to start the proof of (4.2) and quite independent of the rest. The proof is very similar to that of [2, Theorem 2], so we only sketch the main steps. Set

$$
D:=\left\{x \in\left(\mathbb{Z}^{*}\right)^{2}: d(x,(1-\delta) L \mathscr{D}) \leq 1\right\}, \quad D^{\prime}:=\left(\mathbb{Z}^{*}\right)^{2} \cap\left(\left(1+\delta^{3}\right) L \mathscr{D}\right)^{c}
$$

and consider a modified dynamics $(\tilde{\sigma}(t))_{t \geq 0}$ (whose law is denoted $\tilde{\mathbf{P}}$ ), with the same initial condition as $(\sigma(t))_{t \geq 0}$ and the rules that: (i) after each update, any "-" spin which has more than two "+" neighbors is turned to "+", and the operation is repeated as long as such spins exist; (ii) the dynamics stops at the time $\tilde{\tau}_{D, D^{\prime}}$, the first time when there is either a "+" spin in $D$ or a "-" spin in $D^{\prime}$. We define also $\tilde{\tau}_{D}$ the first time when there is a "+" spin in $D$ and $\tau_{D, D^{\prime}}, \tau_{D}$ the analogous random times for the original dynamics.

Note that, by (4.3), w.h.p. $\tau_{D, D^{\prime}}=\tau_{D}$. Note also that the two dynamics can be coupled in a way that $\tau_{D, D^{\prime}}=\tau_{D}$ implies $\tilde{\tau}_{D, D^{\prime}}=\tilde{\tau}_{D} \leq \tau_{D}$ (thanks to point (i) above, since before $\tilde{\tau}_{D, D^{\prime}}$ the modified dynamics has less "-" spins that the original one). Therefore,

$$
\mathbf{P}\left(\tau_{D} \leq \varepsilon L^{2}\right)=\mathbf{P}\left(\tau_{D} \leq \varepsilon L^{2} ; \tau_{D, D^{\prime}}=\tau_{D}\right)+o(1) \leq \tilde{\mathbf{P}}\left(\tilde{\tau}_{D} \leq \varepsilon L^{2} ; \tilde{\tau}_{D, D^{\prime}}=\tilde{\tau}_{D}\right)+o(1)
$$

and it suffices to prove for instance that

$$
\tilde{\mathbf{P}}\left(\tilde{\tau}_{D, D^{\prime}} \leq \varepsilon L^{2} ; \tilde{\tau}_{D}=\tilde{\tau}_{D, D^{\prime}}\right) \leq \exp (-\gamma L)
$$

for some $\varepsilon=\varepsilon(\delta)>0, \gamma>0$. For this, one first observes (as in [2, Eq. (8.6)]) that when $\tilde{\tau}_{D, D^{\prime}}=\tilde{\tau}_{D}$ the difference between the number of "+" spins at time $\tilde{\tau}_{D}$ and the number of "+" spins at time 0 is at least $c \delta^{2} L^{2}$ deterministically, for some $c>0$.

Finally, (as in [2, Eq. (8.10)]) one proves that

$$
\tilde{\mathbf{P}}\left(\left|\left\{x: \tilde{\sigma}_{x}\left(\varepsilon L^{2}\right)=+\right\}\right|-\left|\left\{x: \tilde{\sigma}_{x}(0)=+\right\}\right| \geq c \delta^{2} L^{2}\right) \leq \exp (-\gamma L)
$$

if $\varepsilon=\varepsilon(\delta)$ is small enough. This is based on the fact (cf. [2, Lemma 8.5]) that, for times smaller than $\tilde{\tau}_{D, D^{\prime}}$, the rate of increase of the number of " +" spins is uniformly bounded by a constant.

4.4.2. Scheme of the proof of (4.2). Given some fixed $\delta>0$, we want to prove that for $\varepsilon>0$ small enough, w.h.p.

$$
(1-(\alpha+\delta) \varepsilon) L \mathscr{D} \subset \mathcal{A}_{L}\left(\varepsilon L^{2}\right)
$$

or equivalently

$$
\sigma_{x}\left(\varepsilon L^{2}\right)=- \text { for every } x \in(1-(\alpha+\delta) \varepsilon) L \mathscr{D} .
$$

Given $\xi$ small enough (depending on $\delta$ ) and $\nu$ small enough (depending on $\xi$ ), we define (cf. Figure 8)

$$
\begin{aligned}
U & :=(1-\nu) \mathscr{D}, \\
A_{1}(\varepsilon) & :=[((1-(\alpha+\delta) \varepsilon) \mathscr{D}) \backslash U] \cap[\xi,+\infty)^{2}, \\
B_{1}(\varepsilon) & :=[((1-(\alpha+\delta) \varepsilon) \mathscr{D}) \backslash U] \cap\left([-\xi, \xi] \times \mathbb{R}^{+}\right) .
\end{aligned}
$$

and $A_{i}, B_{i}, i=2,3,4$ as the images of $A_{1}(\varepsilon), B_{1}(\varepsilon)$ by the rotation of angle $i \frac{\pi}{2}$.

One has

$$
(1-(\alpha+\delta) \varepsilon) \mathscr{D}=U \cup\left(\bigcup_{i=1}^{4} A_{i}\right) \cup\left(\bigcup_{i=1}^{4} B_{i}\right)
$$




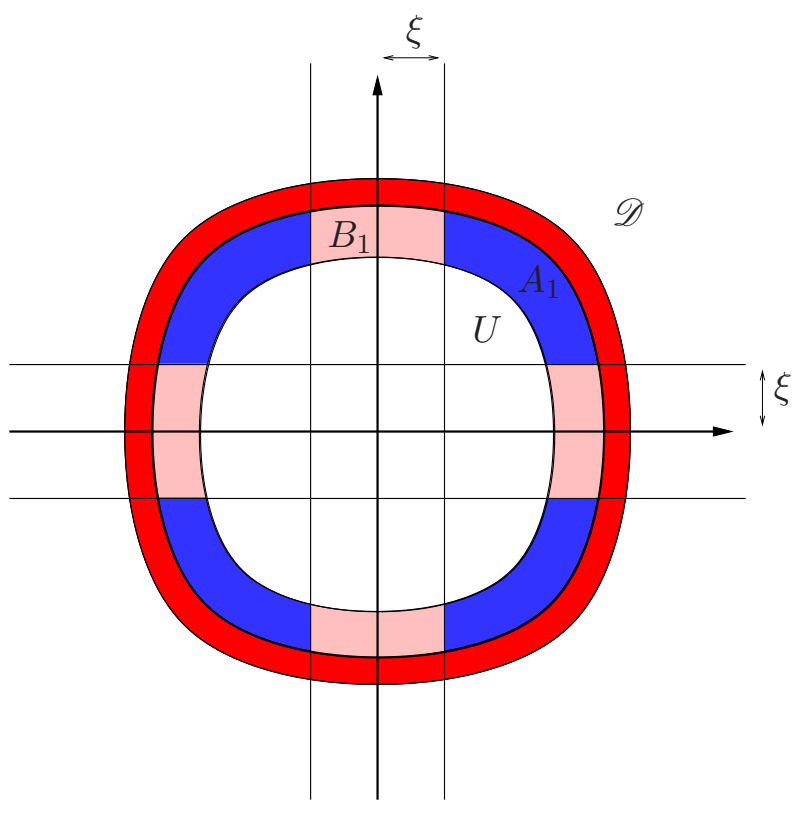

Figure 8. The large droplet is $\mathscr{D}$ and $(1-(\varepsilon(\alpha+\delta))) \mathscr{D}$ is obtained by removing the external dark layer. The white central region $U$, together with $A_{1}, B_{1}$ and its rotations (deformed rectangular regions) form a partition of $(1-\varepsilon(\alpha+\delta)) \mathscr{D}$.

and hence (using rotational symmetries), to prove (4.43), it is sufficient to prove that for $\varepsilon$ small enough, w.h.p.

$$
\begin{aligned}
L U & \subset \mathcal{A}_{L}\left(\varepsilon L^{2}\right), \\
L A_{1}(\varepsilon) & \subset \mathcal{A}_{L}\left(\varepsilon L^{2}\right), \\
L B_{1}(\varepsilon) & \subset \mathcal{A}_{L}\left(\varepsilon L^{2}\right) .
\end{aligned}
$$

The first line, i.e. Equation (4.47), is a direct consequence of (4.4) provided that $\varepsilon$ is chosen small enough (how small depending on $\nu$ ). Actually, one has the following stronger statement that will be useful for what follows: if $\varepsilon$ is small then w.h.p.

$$
L U \subset \mathcal{A}_{L}\left(t L^{2}\right) \text { for every } t \leq \varepsilon .
$$

The main work is thus to prove (4.48) and (4.49).

4.4.3. Proof of (4.49). This is similar to the proof of (4.18), except that monotonicities will be needed in the opposite direction.

Let $\bar{h}:[-2 \xi, 2 \xi] \mapsto \mathbb{R}$ be a concave, twice differentiable, even function such that

$$
\begin{array}{ll}
\bar{h}(x)=h_{0}(x), & \forall x \in[-\xi, \xi], \\
\bar{h}(x)<h_{0}(x), & \forall x \in[-2 \xi,-\xi) \cup(\xi, 2 \xi]
\end{array}
$$

where $h_{0}(\cdot)$ was defined in Section 4.3.2 to be the graph of $\partial \mathscr{D} \cap\left(\mathbb{R} \times \mathbb{R}^{+}\right)$in the $\left(\mathbf{e}_{1}, \mathbf{e}_{2}\right)$ coordinate system. Once $\xi$ is fixed, we choose $\nu$ and $\bar{h}$ such that the point $(2 \xi, \bar{h}(2 \xi))$ lies in the interior of $U$.

Using equation (4.50), we can freeze the spins with vertical coordinate $L \bar{h}(2 \xi)$ and horizontal coordinate in $(-2 L \xi, 2 L \xi)$ (we assume for notational convenience that $2 L \xi$ and $L \bar{h}(2 \xi)$ are in 
$\left.\mathbb{Z}^{*}\right)$ to their initial value "-", and w.h.p., the dynamics we obtain is identical to the original one up to time $\varepsilon L^{2}$.

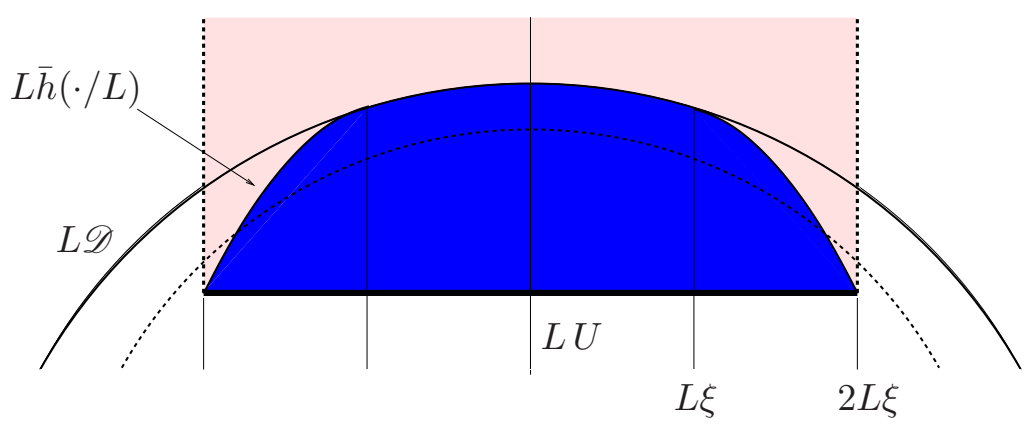

Figure 9. The sites in the dashed vertical lines are frozen to " +" and those of the horizontal bold segment to " -" so that the dynamics in the colored rectangle is independent of the rest of the system. At time $t=0$ the sites in the darkcolored region (whose upper boundary is determined by $\bar{h}(\cdot)$ are " -" while those of the light-colored one are " + ". The function $\bar{h}(\cdot)$ is such that the base of the dark-colored region is in $L U$.

Next we use a chain of monotonicities based on the graphical construction of Section 2.3. Since we are after a lower bound on the set of minuses, we can freeze to "+" all the spins with horizontal coordinate $\pm 2 L \xi$ and vertical coordinate larger than $L \bar{h}(2 \xi)$. Once this is done, we are reduced to considering the dynamics restricted to the set

$$
Y_{2}:=[-2 L \xi+1,2 L \xi-1] \times[L \bar{h}(2 \xi)+1, \infty),
$$

as spins on its boundary are fixed. In principle, the initial condition one should consider is such that $\left(x_{1}, x_{2}\right) \in Y_{2}$ has spin " -" iff $x_{2} \in\left[L \bar{h}(2 \xi)+1, L h^{0}\left(x_{1} / L\right)\right]$, but again by monotonicity, we can add extra "+" spins: we stipulate that, at time $t=0,\left(x_{1}, x_{2}\right)$ has spin " -" iff $x_{2} \in$ $\left[L \bar{h}(2 \xi)+1, L \bar{h}\left(x_{1} / L\right)\right]$. With some abuse of notation, the dynamics thus modified is still called $(\sigma(t))_{t \geq 0}$.

As for the proof of (4.18), we need a final step to map the dynamics onto the interface dynamics of Theorem 3.4, the problem being exactly the same as then: it is not true that the boundary of $\mathcal{A}_{L}(t)$ stays connected for all $t$. The solution adopted in the previous section (leading to the dynamics $\left(\sigma^{(2)}(t)\right)_{t}$, see discussion before (4.35)) does not work here as we are now looking for a lower bound.

Let $\left(\sigma^{(3)}(t)\right)_{t}$ be the dynamics that evolves like $(\sigma(t))_{t}$ except that any spin that has three "+" neighbors is turned instantaneously to "+" (see Figure 10). The coupling given by graphical construction implies that

$$
\bigcup_{\left\{x: \sigma_{x}^{(3)}(t)=-\right\}} \mathcal{C}_{x}=: \mathcal{A}_{L}^{(3)}(t) \subset \mathcal{A}_{L}(t) .
$$

Moreover our choice of initial condition guarantees that $\mathcal{A}_{L}^{(3)}(t)$ stays connected for all time, since the set $\mathscr{D}$ is convex.

We denote by $H_{L}(\cdot, t)$ the càdlàg function $[-2 \xi, 2 \xi] \mapsto \mathbb{R}$ whose graph corresponds to the intersection between $\partial \mathcal{A}_{L}^{(3)}(t)$ and the vertical strip $[-2 L \xi+1 / 2,2 L \xi-1 / 2] \times \mathbb{R}$. Note that 


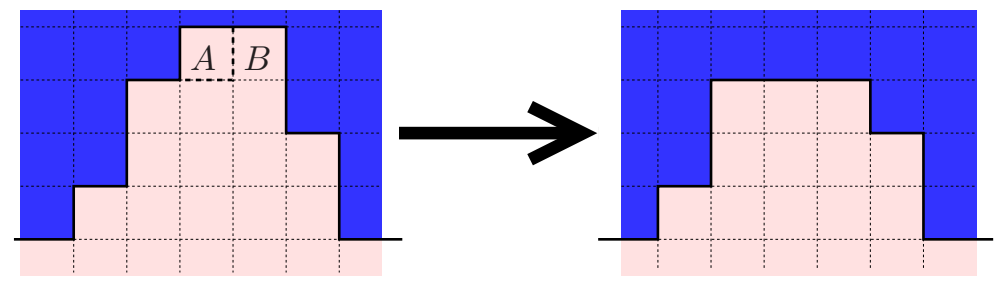

FiguRE 10. Light-colored (resp. dark-colored) squares denote "_" (resp. "+") spins. In our modified dynamics $\sigma^{(3)}$, when a spin has three "+" neighbors, it is instantaneously turned to "+". On the figure, if spin at $A$ is updated and turns to "+", then the spin $B$ has three "+" neighbors and therefore also turns instantaneously to "+".

$H_{L}(\cdot, t)$ can be visualized as a collection of columns of width 1 and integer height. With this notation and (4.53), equation (4.49) is proved if one has w.h.p.

$$
\left.\frac{1}{L} H_{L}\left(x, L^{2} \varepsilon\right) \geq h((\alpha+\delta) \varepsilon, x / L)\right) \text { for every } x \in(-\xi L, \xi L) .
$$

Now we want to relate the dynamics of $H_{L}$ to that of Theorem 3.4. The relation is almost identical to that discussed in Remark 3.3, except for a slight difference in the way particles of types $A$ and $B$ annihilate in the zero-range process. Given $\mathbb{Z} \ni x=-2 L \xi+1 / 2, \ldots, 2 L \xi-1 / 2$, we say again that there are $n>0$ particles of type A at time $t$ at site $x$ if $\lim _{y \rightarrow x^{+}} H_{L}(y, t)-$ $\lim _{y \rightarrow x^{-}} H_{L}(x, t)=n$ and that there are $n>0$ particles of type $\mathrm{B}$ if the same difference equals $-n$. Then it is easy to realize that, under the dynamics $\left(\sigma^{(3)}(t)\right)_{t \geqslant 0}$, each particle performs a symmetric simple random walk with jump rate $1 /(2 n)$ both to right or left (with $n$ the occupation number of the site where the particle is), and that particles of different type annihilate immediately if they are at sites of distance 1 (and not on the same site): this is the effect of flipping instantaneously " -" spins with more than two "+" neighbors. Note also that, due to convexity of $\bar{h}(\cdot)$, particles of type A are always to the left of particles of type B. Therefore, if we take $H_{L}(\cdot, t)$ and we eliminate one of the columns of maximal height (see Figure 11) (note that there are always at least two), the modified height function thus obtained follows exactly the evolution of Theorem 3.4.

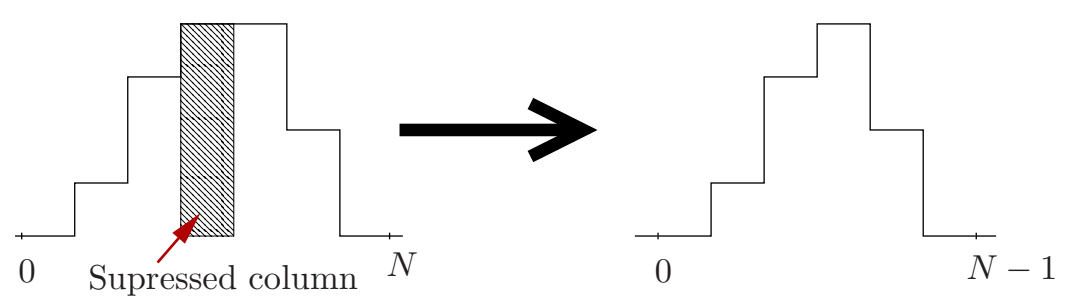

FIGURE 11. Left: the height function associated to the "+/-" boundary for the dynamics $\sigma^{(3)}(t)$. Right: the same height function, with one of the highest columns removed; this follows the same evolution as in Theorem 3.4. The fact that the new interface is step shorter makes no difference in the macroscopic limit. 
Of course, the erased column does not change the scaling limit so that one can apply Theorem 3.4 and Corollary 3.5 and get that for any $t$ and $\eta>0$, w.h.p.

$$
\frac{1}{L} H_{L}\left(x, L^{2} t\right) \geq \bar{\phi}(x / L, t)-\eta \text { for every } x \in\{-2 \xi L, \ldots, 2 \xi L\}
$$

where

$$
\begin{cases}\partial_{t} \bar{\phi}(x, t) & =\frac{1}{2} \partial_{x}^{2} \bar{\phi}(x, t) \\ \bar{\phi}(2 \xi, t) & =\bar{\phi}(-2 \xi, t)=\bar{h}(2 \xi) \\ \bar{\phi}(x, 0) & =\bar{h}(x) \quad \text { for every } x \in[-2 \xi, 2 \xi] .\end{cases}
$$

Therefore, (4.54) is proved if one can check that

$$
\bar{\phi}(x, \varepsilon)>h(x,(\alpha+\delta) \varepsilon) \text { for every } x \in[-\xi, \xi] .
$$

The above equation is proved is the same manner as (4.39): one just needs to choose $\xi$ small enough.

4.4.4. Proof of (4.48). First of all, one freezes to "-" all the spins on the cross-shaped region of sites in $L U$ (cf. (4.45)) such that at least one of their coordinates is $\pm 1 / 2$. Equation (4.50) guarantees that if $\varepsilon$ is chosen small enough, w.h.p. the so-obtained dynamics coincides with the original one up to time $\varepsilon L^{2}$ if $\varepsilon$ is small enough.

Then one defines $\left(\sigma^{(4)}(t)\right)_{t \geqslant 0}$ as the dynamics obtained by changing the initial condition in the following manner: all spins $(x, y) \in L \mathscr{D}$ with either $|x| \geqslant L(1-\nu)$ or $|y| \geqslant L(1-\nu)$ are changed from "-" to " +" (recall that $\nu$ is the constant that enters the definition (4.45) of $U$ ) and therefore they stay "+" forever, since they have at least three "+" neighbors. Note that, this way, the evolution in each quadrant of $\left(\mathbb{Z}^{*}\right)^{2}$ is independent. By monotonicity, we get that w.h.p, for every $t \leq \varepsilon L^{2}$,

$$
\bigcup_{\left\{x: \sigma_{x}^{(4)}(t)=-\right\}} \mathcal{C}_{x}=: \mathcal{A}_{L}^{(4)}(t) \subset \mathcal{A}_{L}(t) .
$$

and therefore (4.48) is proved if one can show that

$$
L A_{1}(\varepsilon) \subset \mathcal{A}_{L}^{(4)}\left(\varepsilon L^{2}\right) .
$$

Next, note that $\partial \mathcal{A}_{L}^{(4)}(t) \cap \mathbb{R}_{+}^{2}$ in the coordinate system $\left(\mathbf{f}_{1}, \mathbf{f}_{2}\right)$ is the graph of a random piecewise linear function

$$
F_{L}:\left[-\frac{(1-\nu) L}{\sqrt{2}}, \frac{(1-\nu) L}{\sqrt{2}}\right] \mapsto \mathbb{R}
$$

which undergoes the corner-flip dynamics described in Theorem 3.2 (apart from space rescaling by a factor $\sqrt{2}$ ). For this reason on gets that, w.h.p.,

$$
\lim _{L \rightarrow \infty} \sup _{x \in\left[-\frac{1-\nu}{\sqrt{2}}, \frac{1-\nu}{\sqrt{2}}\right]} \sup _{t \leqslant \varepsilon}\left|\frac{1}{L} F_{L}\left(x L, t L^{2}\right)-g(x, t)\right|=0
$$

where

$$
\begin{cases}\partial_{t} g(x, t) & =\frac{1}{4} \partial_{x}^{2} g(x, t) \\ g\left(-\frac{1-\nu}{\sqrt{2}}, t\right) & =g\left(\frac{1-\nu}{\sqrt{2}}, t\right)=\frac{1-\nu}{\sqrt{2}} \\ g(x, 0) & =\bar{f}(x) \quad \text { for every } x \in\left[\frac{1-\nu}{\sqrt{2}}, \frac{1-\nu}{\sqrt{2}}\right]\end{cases}
$$

and $\bar{f}$ is the profile of the initial condition, i.e.

$$
\left.\bar{f}(x):=\min \left(f_{0}(x),(1-\nu) \sqrt{2}-|x|\right)\right) .
$$


Let $p$ (resp. $p_{1}$ ) be the point on $\partial \mathscr{D}$ whose coordinates $(x, y)$ (resp. $\left.\left(x_{1}, x_{2}\right)\right)$ in the coordinate system $\left(\mathbf{e}_{1}, \mathbf{e}_{2}\right)$ satisfy $x>0, y=1-\nu\left(\right.$ resp. $\left.x_{1}>0, y_{1}=h_{0}(\xi)\right)$. Call $-d<0\left(\right.$ resp. $\left.-d_{1}<0\right)$ the horizontal coordinate of $p$ (resp. of $p_{1}$ ) in the coordinate system $\left(\mathbf{f}_{1}, \mathbf{f}_{2}\right)$.

In view of (4.60) and of Definition (4.45) of $A_{1}(\varepsilon)$, equation (4.59) is satisfied if

$$
g(x, \varepsilon)>f(x,(\alpha+\delta) \varepsilon) \text { for every } \quad x \in\left(-d_{1}, d_{1}\right),
$$

The proof of this is very similar to that of (4.24) provided that that $\bar{f}$ coincides with $f_{0}$ in a domain containing strictly $\left(-d_{1}, d_{1}\right)$ (this guarantees for instance that $\partial_{x}^{2} \bar{f}(\cdot)$ is uniformly continuous in a domain containing $\left(-d_{1}, d_{1}\right)$, so that the drift $\partial_{t} g$ is continuous in time, cf. Lemma 4.3). For this to hold, it is enough to assume that $d>d_{1}$, i.e. that $\nu$ in (4.45) has been chosen sufficiently small as a function of $\xi$ so that $1-\nu>h_{0}(\xi)$.

\section{Proof of Theorem 2.1: existence of Anisotropic CURVE-Shortening Flow With CONVEX INITIAL CONDITION}

Let us first recall some properties of the support function $h(\cdot)$ of a convex curve $\gamma$. First of all, if $\gamma \subset \gamma^{\prime}$ then $h(\theta) \leq h^{\prime}(\theta)$ for every $\theta$. Next, the support function is related to the curvature and to the length $L(\gamma)$ of $\gamma$ by (cf. [12, Lemma 1.1])

$$
\begin{gathered}
\partial_{\theta}^{2} h(\theta)+h(\theta)=\frac{1}{k(\theta)} \\
L(\gamma)=\int_{0}^{2 \pi} h(\theta) \mathrm{d} \theta=\int_{0}^{2 \pi} \frac{1}{k(\theta)} \mathrm{d} \theta .
\end{gathered}
$$

Also (cf. Lemma 4.1.1 in [13], with the warning that what they call $\theta$ is $\theta-\pi / 2$ for us), the Cartesian coordinates $(x(\theta), y(\theta))$ of the point of $\gamma$ where the outward directed normal forms an anticlockwise angle $\theta$ with the positive horizontal axis can be expressed as

$$
\begin{gathered}
x(\theta)=h(0)-\int_{0}^{\theta} \frac{\sin (s)}{k(s)} d s \\
y(\theta)=h(\pi / 2)+\int_{\pi / 2}^{\theta} \frac{\cos (s)}{k(s)} d s .
\end{gathered}
$$

Under the flow (2.7), the time derivatives of area and length are (cf. [12, Lemma 2.1])

$$
\begin{gathered}
\frac{d}{d t} \operatorname{Area}(\gamma(t))=-\int_{0}^{2 \pi} a(\theta) \mathrm{d} \theta \\
\frac{d}{d t} L(\gamma(t))=-\int_{0}^{2 \pi} a(\theta) k(\theta, t) \mathrm{d} \theta .
\end{gathered}
$$

For the moment these are formal statements since we do not know yet that the flow exists.

5.1. Proof of Theorem 2.1. Uniqueness of the flow is trivial, so we concentrate on existence. First of all, we need to regularize the functions $a(\cdot)$ and $k(\cdot)$. Given $0<w<1$ we define $a^{(w)}(\cdot)$ to be a family of smooth approximations of the anisotropy function $a(\cdot)$. More precisely:

\section{Assumption 5.1.}

(1) $a^{(w)}(\cdot)$ is $2 \pi$-periodic and $C^{\infty}$;

(2) $a^{(w)}(\theta) \stackrel{w \rightarrow 0}{\longrightarrow} a(\theta)$ uniformly in $\theta$;

(3) for fixed $\theta$, the function $w \mapsto a^{(w)}(\theta)$ is non-increasing;

(4) the function $a^{(w)}(\cdot)$ is Lipschitz, uniformly in $w>0$ (this is possible because the function $a(\cdot)$ itself is 1-Lipschitz); 
(5) the functions $w \mapsto\left\|\partial_{\theta}^{2} a^{(w)}\right\|_{\infty}:=\max _{\theta}\left|\partial_{\theta}^{2} a^{(w)}(\theta)\right|$ and $w \mapsto\left\|\partial_{\theta}^{3} a^{(w)}\right\|_{\infty}$ are bounded, uniformly for $w$ in any compact subset of $(0,1)$.

A possible choice is

$$
a^{(w)}(\theta)=\left(a * g^{(w)}\right)(\theta)+\varepsilon_{w}
$$

where $g^{(w)}$ is a centered Gaussian of variance $w^{2}$. In the convolution it is understood that $a(\cdot)$ is seen as a $2 \pi$-periodic function on $\mathbb{R}$ and $\varepsilon_{w}$ is chosen so that $a^{(w)}(\cdot)$ satisfies the monotonicity with respect to $w$. It is easy to check that one can choose $\varepsilon_{w}=-C w$ for some suitably large $C$. Indeed, monotonicity in $w$ is guaranteed if for $w^{\prime}<w$ one has

$$
\varepsilon_{w^{\prime}}-\varepsilon_{w} \geq\left\|a *\left(g^{(w)}-g^{\left(w^{\prime}\right)}\right)\right\|_{\infty} .
$$

On the other hand, since $a(\cdot)$ is Lipschitz, one sees easily that $\left\|a *\left(g^{(w)}-g^{\left(w^{\prime}\right)}\right)\right\|_{\infty}=O\left(w-w^{\prime}\right)$.

Also, we approximate $\gamma$ with a sequence of convex curves $\left(\gamma^{(w)}\right)_{0<w<1}$ that satisfy the following properties:

\section{Assumption 5.2.}

(1) $\gamma^{(w)} \supset \gamma^{\left(w^{\prime}\right)} \supset \gamma$ or equivalently $h^{(w)}(\cdot) \geq h^{\left(w^{\prime}\right)}(\cdot) \geq h(\cdot)$ if $0<w^{\prime}<w$;

(2) $\lim _{w \rightarrow 0} h^{(w)}(\cdot)=h(\cdot)$ uniformly in $\theta$, so that $\gamma$ is the limit of $\gamma^{(w)}$ in the topology of the Hausdorff distance;

(3) the Lipschitz constant $\mathbb{L}\left(k^{(w)}\right)$ of the curvature function $k^{(w)}(\cdot)$ is finite uniformly in $w$, $k^{(w)}(\cdot) \rightarrow k(\cdot)$ uniformly and $\lim _{\sup _{w \rightarrow 0}} \mathbb{L}\left(k^{(w)}\right) \leq \mathbb{L}(k) ;$

(4) The three first derivatives with respect to $\theta$ of $k^{(w)}(\theta)$ are bounded uniformly for $w$ in any compact subset of $(0,1)$.

(Like for the regularization of $a(\cdot)$ into $a^{(w)}(\cdot)$, a possible construction of $h^{(w)}(\cdot)$ is obtained convolving $h(\cdot)$ with a Gaussian of variance $w^{2}$ and adding a suitable constant $\left.\varepsilon_{w}\right)$.

For the regularized mean curvature motion, it follows from [12] that the equation

$$
\left\{\begin{array}{l}
\partial_{t} h^{(w)}(\theta, t)=-a^{(w)}(\theta) k^{(w)}(\theta, t) \\
h^{(w)}(\theta, 0)=h^{(w)}(\theta)
\end{array}\right.
$$

admits a solution corresponding to a flow of curves $\left(\gamma^{(w)}(t)\right)_{t \geq 0}$ which remain convex and shrink to a point in a finite time

$$
\tilde{t}_{f}:=t_{f}^{(w)}=\operatorname{Area}\left(\gamma^{(w)}(0)\right) / \int_{0}^{2 \pi} a^{(w)}(\theta) \mathrm{d} \theta
$$

(cf. (5.5) with $a(\cdot)$ replaced by $a^{(w)}(\cdot)$ ). For lightness of notation, we will often write $\tilde{h}(\cdot, \cdot), \tilde{\gamma}(t), \tilde{a}(\cdot)$, etc. for the regularized quantities $h^{(w)}(\cdot, \cdot), \gamma^{(w)}(t), a^{(w)}(\cdot)$, etc. Also, we abusively identify a convex curve $\gamma$ with the set enclosed by it. Thanks to Assumption 5.1, we have that $\int_{0}^{2 \pi} a^{(w)}(\theta) \mathrm{d} \theta \rightarrow \int_{0}^{2 \pi} a(\theta) \mathrm{d} \theta=2$ as $w \rightarrow 0$ and therefore $t_{f}^{(w)}=t_{f}(1+o(1))$ with $t_{f}$ defined in Theorem 2.1 when $w \rightarrow 0$.

From (5.1) and (5.7) one can check that the curvature satisfies the parabolic equation

$$
\left\{\begin{array}{l}
\partial_{t} \tilde{k}=\tilde{k}^{2} \partial_{\theta}^{2}(\tilde{a} \tilde{k})+\tilde{a} \tilde{k}^{3} \\
\tilde{k}(\theta, 0)=\tilde{k}(\theta) .
\end{array}\right.
$$

Also, following [13] it is possible to see that the curvature function stays $C^{\infty}$ until $\tilde{t}_{f}$ (since $\tilde{a}$ is $\left.C^{\infty}\right)$. However, estimates on the regularity will not be necessarily uniform in the regularization parameter $w$ and we will need to be very careful on this point. 
For fixed $t$, let

$$
\gamma(t)=\lim _{w \rightarrow 0} \gamma^{(w)}(t)
$$

where convergence is in the Hausdorff metric. A posteriori, since we will see that $(\gamma(t))_{t}$ provides the (unique) solution to our curve-shortening equation, it follows that the limit (5.9) does not depend on the choice of regularization. Existence of the limit is guaranteed by the fact that $\gamma^{\left(w^{\prime}\right)}(t) \subset \gamma^{(w)}(t)$ if $w^{\prime}<w$ and $t<t_{f}^{\left(w^{\prime}\right)}$ (this follows from the fact that $a^{(w)}(\theta)$ is decreasing in $w$ and that the curve is smooth at all times). One has to use the Blaschke selection theorem [8, Th. 32] which says that a family of convex subsets of a bounded subset of $\mathbb{R}^{n}$ admits a sub-sequence converging to a non-empty convex set. Convergence in Hausdorff distance also holds for the boundary curves.

Since the volume is continuous in the topology induced by the Hausdorff metric [8, Ch. 4] we also see that $\operatorname{Area}(\gamma(t))=\operatorname{Area}(\gamma)-t \int_{0}^{2 \pi} a(\theta) \mathrm{d} \theta=\operatorname{Area}(\gamma)-2 t$; for $t \rightarrow t_{f}$ the curve $\gamma(t)$ shrinks to a point (its diameter shrinks to zero).

Definition 5.1. For $t<\tilde{t}_{f}$ let $\tilde{k}_{\max }(t)$ (resp. $\tilde{k}_{\min }(t)$ ) be the maximal (resp. minimal) curvature of $\tilde{\gamma}(t)$. We let $\tilde{k}_{\max }:=\tilde{k}_{\max }(0)$ and similarly for $\tilde{k}_{\min }$ and $\tilde{a}_{\max (\min )}:=\max _{\theta}\left(\min _{\theta}\right) \tilde{a}(\theta)$. Also, $k_{\min (\max )}$ and $a_{\min (\max )}$ are defined similarly to $\tilde{k}_{\min (\max )}, \tilde{a}_{\min (\max )}$ but with $\tilde{k}(\cdot), \tilde{a}(\cdot)$ replaced by $k(\cdot), a(\cdot)$.

It is crucial that $\tilde{k}_{\max }(t)$ stays bounded, uniformly for $w$ small, as long as the disappearance time is not approached:

Proposition 5.2 (Regularity estimate). Assume that the curvature function $k(\cdot)$ is Lipschitz. There exists $w_{0}>0$ such that, for every $b>0, t<t_{f}(1-b), 0<w \leq w_{0}$ one has

$$
\tilde{k}_{\max }(t) \leq C_{1}
$$

and

$$
\max _{\theta}\left|\partial_{\theta}(\tilde{a}(\theta) \tilde{k}(\theta, t))\right| \leq C_{2}+\mathbb{L}(k)
$$

where we recall that $\mathbb{L}(k)$ is the Lipschitz constant of the function $k(\cdot)$. The constants $C_{1}$ and $C_{2}$ depend only on $b$ and on $k_{\max }$.

We will prove

Theorem 5.3. The flow of curves $(\gamma(t))_{t<t_{f}}$ defined in (5.9) is a classical solution of the anisotropic curve shortening flow (2.7) for $0 \leq t<t_{f}$.

Proof of Proposition 5.2. The proof is based on ideas of [12]. However, it is important to make sure that estimates are uniform in $w \leq w_{0}$ (in [12] the anisotropy function $a(\cdot)$ is assumed to be $C^{2}$, so there was no need to regularize it).

Fix $w>0$. First we get a lower bound on $\tilde{k}_{\min }(t)$. Note first of all that at time zero the minimal curvature is bounded away from zero (uniformly in $w$ ): indeed, using (5.2) and the fact that the curvature function is $\mathbb{L}(k)$-Lipschitz,

$$
L(\gamma(0))=\int_{0}^{2 \pi} \frac{1}{k(\theta)} \mathrm{d} \theta \geq 2 \int_{0}^{\pi} \frac{1}{k_{\min }+\mathbb{L}(k) \theta} \mathrm{d} \theta=\frac{2}{\mathbb{L}(k)} \log \frac{\mathbb{L}(k) \pi+k_{\min }}{k_{\min }} .
$$

Then, since the length of $\gamma(0)$ is finite, $k_{\min }$ must be positive.

Set for simplicity

$$
g=g(\theta, t)=\tilde{a}(\theta) \tilde{k}(\theta, t)
$$


Formula (5.8) gives

$$
\partial_{t} g=\frac{1}{\tilde{a}}\left(g^{2} \partial_{\theta}^{2} g+g^{3}\right)=: g(\theta, t) u(\theta, t) .
$$

This, together with the fact that $\tilde{a}(\cdot)$ and $\tilde{k}(\cdot, t)$ are smooth, implies that

$$
\frac{d}{d t} \min _{\theta} g \geq \frac{\min _{\theta} g^{3}}{\tilde{a}_{\max }} \geq 0
$$

(at the minimum point the second derivative is positive) so that

$$
\tilde{k}_{\min }(t) \geq \frac{\tilde{a}_{\min }}{\tilde{a}_{\max }} \tilde{k}_{\min } \geq C k_{\min }>0
$$

with $C$ independent of $w$ (say for $w \leq w_{0}$ ) thanks to the uniform convergence $a^{(w)}(\cdot) \rightarrow a(\cdot)$ and $k^{(w)}(\cdot) \rightarrow k(\cdot)$.

Next the real work: bounding $\tilde{k}_{\max }(t)$ uniformly in $w$. From $(5.13)$ one sees that, since $\tilde{a}(\cdot)$ and $\tilde{k}(\cdot, t)$ are smooth,

$$
\frac{d}{d t} \max _{\theta} g \leq \frac{1}{\tilde{a}_{\min }} \times\left(\max _{\theta} g\right)^{3} .
$$

From this one immediately gets that $\tilde{k}_{\max }(t)$ is upper bounded uniformly in $w \leq w_{0}$, up to some time $t_{1}$ depending only on $k_{\max }$. However the solution of $\dot{x}=x^{3}$ explodes in finite time, certainly before the time $\tilde{t}_{f}$ when the curve shrinks to a point, so we need to do better.

For this, we define $z(t)=\min _{\theta} u(\theta, t)$ (cf. (5.13)). Then, taking the derivative of $u$ with respect to $t$ shows (cf. Lemma 4.2 of [12] for details) that

$$
\frac{\mathrm{d}}{\mathrm{d} t} z(t) \geq 2 z(t)^{2}
$$

so that if $z(0) \geq 0$ we get $z(t) \geq 0$, if $z(0) \leq 0$ we get $z(t) \geq-1(1 /|z(0)|+2 t)$. Altogether, we get that

$$
u(\theta, t) \geq-\frac{1}{2 t}
$$

uniformly in $\theta$ and $w \leq w_{0}$. Now we use this to get a uniform bound on $\left\|\partial_{\theta} g\right\|_{\infty}$ in terms of $\tilde{k}_{\max }(t)$. Without loss of generality suppose that there exists $\theta_{1}$ such that $\partial_{\theta} g\left(\theta_{1}, t\right)=\left\|\partial_{\theta} g\right\|_{\infty}$ (if this is not the case one can still find $\theta_{1}$ such that $\partial_{\theta} g\left(\theta_{1}, t\right)=-\left\|\partial_{\theta} g\right\|_{\infty}$ and apply the same method). Let also $\theta_{2}>\theta_{1}$ be such that $\partial_{\theta} g\left(\theta_{2}, t\right)=0$ (such angle exists since $g$ is periodic). Then, from the definition (5.13) of $u$,

$$
\begin{aligned}
&\left\|\partial_{\theta} g\right\|_{\infty}=-\int_{\theta_{1}}^{\theta_{2}} \partial_{\theta}^{2} g \mathrm{~d} \theta=-\int_{\theta_{1}}^{\theta_{2}}\left(\frac{u(\theta, t)}{\tilde{k}(\theta, t)}-\tilde{a}(\theta, t) \tilde{k}(\theta, t)\right) \mathrm{d} \theta \\
& \leq \frac{1}{2 t} \int_{\theta_{1}}^{\theta_{2}} \frac{\mathrm{d} \theta}{\tilde{k}(\theta, t)}+\left(\theta_{2}-\theta_{1}\right) \tilde{a}_{\max } \tilde{k}_{\max }(t) \leq \frac{L(\gamma(0))}{t}+C_{4} \tilde{k}_{\max }(t) .
\end{aligned}
$$

In the last inequality we used (5.2) and then (5.6) which says that $L(\tilde{\gamma}(t)) \leq L(\tilde{\gamma}(0))(\leq$ $2 L(\gamma(0)))$. Since $g=\tilde{a} \tilde{k}$ and by assumption $\tilde{a}$ is $C^{\infty}$ and Lipschitz uniformly in $w$, one deduces that

$$
\left\|\partial_{\theta} \tilde{k}\right\|_{\infty} \leq \frac{L(\gamma(0))}{t}+C_{5} \tilde{k}_{\max }(t) \leq C_{6}(t) \tilde{k}_{\max }(t)
$$

and $C_{6}$ can be chosen to be decreasing in $t$. From this it is trivial to see that, if $\theta_{0}$ is such that $\tilde{k}\left(\theta_{0}, t\right)=\tilde{k}_{\max }(t)$, one has

$$
\tilde{k}(\theta, t) \geq \tilde{k}_{\max }(t) / 2 \text { whenever }\left|\theta-\theta_{0}\right| \leq \alpha(t)
$$


for some $\alpha(t)$ increasing in $t$ (it could vanish for $t \rightarrow 0$ ). Next, one proves that for $t<(1-b) t_{f}$

$$
E(t):=\int_{0}^{2 \pi} \tilde{a}(\theta) \log (g(\theta, t)) \mathrm{d} \theta \leq C_{7}
$$

where $C_{7}$ depends only on $a_{\max }$ and on $b$ and on the maximal curvature $k_{\text {max }}$ of the initial curve $\gamma(0)$. Indeed, (5.21) is obvious for $t=0$, since the initial curvature is bounded by assumption. To get the control for $t>0$, one observes (cf. Propositions 5.3 and 5.4 of [12]) that

$$
\frac{\mathrm{d}}{\mathrm{d} t} E(t) \leq 2 \tilde{a}_{\max } \frac{L(\tilde{\gamma}(0))}{\operatorname{Area}\left(\tilde{\gamma}\left((1-b) t_{f}\right)\right)}\left(-\frac{\mathrm{d}}{\mathrm{d} t} L(\tilde{\gamma}(t))\right) .
$$

The prefactor is bounded since $b>0$ and the time-integral of the time-derivative of the length gives at most $L(\tilde{\gamma}(0))$. At this point we are almost done: using (5.20)

$$
\begin{gathered}
C_{7} \geq \int_{0}^{2 \pi} \tilde{a}(\theta) \log (g(\theta, t)) \mathrm{d} \theta \\
\geq 2 \alpha(t) \tilde{a}_{\min } \log \left(\tilde{a}_{\min } \tilde{k}_{\max }(t) / 2\right)+2 \pi \tilde{a}_{\max } \log \left[\min \left(1, \tilde{a}_{\min } \tilde{k}_{\min }(t)\right)\right]
\end{gathered}
$$

and this (recall that $\tilde{k}_{\min }(t) \geq C k_{\min }>0$, cf. (5.14)) gives us an upper bound on $\tilde{k}_{\max }(t)$ uniformly in $w \leq w_{0}$ and $t<(1-b) t_{f}$ : up to $t_{1}$ one uses the upper bound which comes from (5.16) and after $t_{1}$ the one from (5.22); Eq. (5.10) is proven. When $t$ approaches the disappearance time $\tilde{t}_{f}$ (i.e. when $b$ approaches zero) the upper bound diverges (because $C_{7}$ diverges), as it should.

Equation (5.19) says that the curvature function is Lipschitz with a Lipschitz constant $C$ that depends on $t, b$ and $L(0)$ but not on $w$. This is not yet the desired (5.11) because the bound diverges for $t \rightarrow 0$. To prove (5.11) remark that, using (5.13),

$$
\begin{aligned}
\partial_{t} \partial_{\theta} g & =\partial_{\theta}\left(\frac{g^{2}}{\tilde{a}} \partial_{\theta}^{2} g+\frac{g^{3}}{\tilde{a}}\right)=-\frac{\partial_{\theta} \tilde{a}}{\tilde{a}^{2}}\left(g^{2} \partial_{\theta}^{2} g+g^{3}\right) \\
& +\frac{1}{\tilde{a}}\left(2 g \partial_{\theta} g \partial_{\theta}^{2} g+g^{2} \partial_{\theta}^{3} g+3 g^{2} \partial_{\theta} g\right) .
\end{aligned}
$$

At the point where $\partial_{\theta} g$ is maximized, $\partial_{\theta}^{2} g$ cancels and $\partial_{\theta}^{3} g$ is non-positive. This, together with the boundedness of $g$ uniformly in $w \leq w_{0}, \theta \in[0,2 \pi]$ and $t<(1-b) t_{f}$, implies

$$
\partial_{t} \max _{\theta} \partial_{\theta} g(\theta, t) \leq C_{8}\left(1+\max _{\theta} \partial_{\theta} g(\theta, t)\right) .
$$

where $C_{8}$ just depends on $k_{\max }$ and $b$. Integrating with respect to time, one gets

$$
\max _{\theta} \partial_{\theta} g(\theta, t) \leq \max _{\theta} \partial_{\theta}\left(a^{(w)}(\theta) k^{(w)}(\theta)\right)+C_{9}
$$

with $C_{9}$ depending only on $C_{8}$. Also, observe that

$$
\partial_{\theta}\left(a^{(w)}(\theta) k^{(w)}(\theta)\right) \leq(3 / 4)\left|\partial_{\theta} k^{(w)}(\theta)\right|+C_{10} \leq(3 / 4) \mathbb{L}\left(k^{(w)}\right)+C_{10}
$$

with $C_{10}$ a constant depending on $k_{\max }$, since for $w$ small $a_{\max }^{(w)}<(3 / 4)$ and $a^{(w)}$ is uniformly Lipschitz. Finally, from Assumption $5.2(3)$, we can conclude $\partial_{\theta}\left(a^{(w)}(\theta) k^{(w)}(\theta)\right) \leq C_{10}+\mathbb{L}(k)$ for $w$ small. An analogous lower bound can be found on $\partial_{t} \min _{\theta} \partial_{\theta} g(\theta, t)$ and this gives (5.11).

Following [13] it is possible to prove that, once we have bounds on curvature and on $\left\|\partial_{\theta} g(\cdot, t)\right\|_{\infty}$, for every $n \geq 2$ and $t<t_{f}(1-b)$ the derivatives $\partial_{\theta}^{n} g(\theta, t)$ are also bounded. The bounds we get are in general not uniform in $w$ but this is not very important for our purposes. Indeed, we will need only: 
Proposition 5.4. Fix $b>0$. There exists a function $c(w)$, which is non-increasing with respect to $w \in\left(0, w_{0}\right]$ such that for $t<(1-b) t_{f}$

$$
\max _{\theta}\left|\partial_{t}^{2} \tilde{h}(\theta, t)\right| \leq c(w)
$$

Proof. Recall (5.7) and (5.13):

$$
\partial_{t}^{2} \tilde{h}=-\frac{1}{\tilde{a}}\left(g^{2} \partial_{\theta}^{2} g+g^{3}\right) .
$$

Thus we just have to bound $\partial_{\theta}^{2} g$, since we have already proved that $g$ itself is bounded. For this, we adapt the method used by Gage and Hamilton in [13] for the special case of the isotropic curve shortening flow where $a \equiv 1$. What they observed [13, Lemma 4.4.2] is that, if the curvature and its $\theta$-derivative are bounded (which we proved in Proposition 5.2), the $t$-derivative of $\Phi(t):=\int_{0}^{2 \pi}\left[\partial_{\theta}^{2} g(\theta, t)\right]^{4} \mathrm{~d} \theta$ can be upper bounded by a constant times $\Phi(t)$ itself and then one can integrate the inequality with respect to $t$ to get a bound on $\Phi(t)$ in terms of $\Phi(0)$. In our case, with a similar computation, we find that $(d / d t) \Phi(t)$ is upper bounded by $\Phi(t)$ times a constant depending on $\left\|\partial_{\theta} a^{(w)}\right\|_{\infty}$, which is finite uniformly for $w \leq 1$. Since $\Phi(0)$ is also bounded for $w$ in compact subsets of $(0,1)$ (cf. Assumption 5.1 (5) and Assumption5.2 (4)), we get that $\Phi(t) \leq c_{1}(w)$ for $w \in(0,1)$ and $t<(1-b) t_{f}$ and we can choose $c_{1}$ to be decreasing. In general, $c_{1}$ will diverges when $w$ approaches zero.

A similar computation (cf. [13, Lemma 4.4.3] when $a(\theta) \equiv 1$ ) shows that

$$
\Psi(t):=\int_{0}^{2 \pi}\left[\partial_{\theta}^{3} g(\theta, t)\right]^{2} \mathrm{~d} \theta \leq c_{2}(w)
$$

with $c_{2}(\cdot)$ decreasing in $w \in(0,1)$. Then one uses the fact that for a smooth, $2 \pi$-periodic function $f$ one has (cf. [13, Corollary 4.4.4])

$$
\|f\|_{\infty}^{2} \leq C \int_{0}^{2 \pi}\left(f^{2}+\left(\partial_{\theta} f\right)^{2}\right) \mathrm{d} \theta
$$

for some universal constant $C$, applied with $f(\cdot)=\partial_{\theta}^{2} g(\cdot, t)$, to get that $\left\|\partial_{\theta}^{2} g\right\|_{\infty} \leq c_{3}(w)$ as we wished.

Proof of Theorem 5.3. We are now ready to prove that $(\gamma(t))_{t}$ provides a classical solution of (2.7). This is based on the following easy consequence of the Arzelà-Ascoli Theorem:

Lemma 5.5. Let $f^{(n)}$ be a sequence of periodic $C^{1}$ functions on $[0,2 \pi]$, such that both sequences $f^{(n)}$ and $\partial_{x} f^{(n)}$ are uniformly bounded and equicontinuous. If $f^{(n)} \rightarrow f$ as $n \rightarrow \infty$, then $f$ is $C^{1}$ and $\partial_{x} f=\lim _{n} \partial_{x} f^{(n)}$, where the convergence is uniform and does not require sub-sequences.

First of all, we note that $\tilde{h}(\cdot, t)$ does converge (for $w \rightarrow 0$ ) to $h(\cdot, t)$ for every fixed $t<t_{f}$. This just follows from the fact that $\tilde{\gamma}(t)$ converges to $\gamma(t)$ in terms of Hausdorff distance. Applying Lemma 5.5 and recalling (5.1), we get that, for $t$ fixed, $\partial_{\theta} \tilde{h}(\theta, t)$ and $\tilde{k}(\theta, t)$ converge to $\partial_{\theta} h(\theta, t)$ and $k(t, \theta)$ respectively and that convergences are uniform in $\theta$ (knowing that the curvature is Lipschitz is important here). Note by the way that $k(\cdot, t)$ is Lipschitz, since $\left\|\partial_{\theta} \tilde{k}(\cdot, t)\right\|_{\infty}$ is uniformly bounded.

Then applying dominated convergence (which is allowed in view of Proposition 5.2), one gets that

$$
h(\theta, t)-h(\theta, s)=-\int_{s}^{t} a(\theta) k(\theta, u) \mathrm{d} u
$$


which is an integrated version of (2.7). To get the stronger statement (2.7), we need to prove that $k(\theta, t)$ is continuous as a function of $t$.

First of all, we prove that one can find a function $\varepsilon:(0,1) \ni w \mapsto \varepsilon(w) \in \mathbb{R}_{+}$, increasing and going to zero as $w \rightarrow 0$ such that for all $\theta$, for all $t \leq(1-b) t_{f}$,

$$
|\tilde{k}(\theta, t)-k(\theta, t)| \leq \varepsilon(w) .
$$

If this were not the case then, thanks to the fact that $\tilde{k}(\cdot, t)$ and $k(\cdot, t)$ are uniformly Lipschitz, we would have, say, for arbitrarily small $w$ and for some $\varepsilon>0$,

$$
\tilde{k}(t, \theta)-k(t, \theta) \geq \varepsilon
$$

for $\theta \in[\bar{\theta}, \bar{\theta}+\varepsilon]$ for some $\bar{\theta} \in[0,2 \pi]$. But then, since (cf. (5.1))

$$
\left(\partial_{\theta}^{2}+1\right)(h(\theta, t)-\tilde{h}(\theta, t))=\frac{1}{k(\theta, t)}-\frac{1}{\tilde{k}(\theta, t)},
$$

this would contradict the uniform convergence of $\tilde{h}(\cdot, t)$ to $h(\cdot, t)$.

On the other hand, from Proposition 5.4, for all $\theta$ and for all $t, s \leq(1-b) t_{f}$

$$
|\tilde{k}(\theta, t)-\tilde{k}(\theta, s)| \leq c(w)|t-s| .
$$

Together with (5.30) this implies that

$$
|k(\theta, t)-k(\theta, s)| \leq \inf _{w}(2 \varepsilon(w)+c(w)|t-s|) .
$$

The right-hand side clearly tends to zero with $|t-s|$ (choose a sequence $\left\{w_{k}\right\}$ tending to zero. If $c\left(w_{k}\right)$ does not diverge we are done. Otherwise, compute the right-hand side for the $w=w_{k}$ with the largest value of $k$ such that $\left.c\left(w_{k}\right) \leq|t-s|^{-1 / 2}\right)$. This shows that $t \mapsto k(\theta, t)$ is continuous away from $t_{f}$ and the proof is complete.

\section{Proof of Theorem 2.2: evolution of A CONVEX Droplet}

The proof is very similar to that of Theorem 2.3 in the scale-invariant case (Section 4), and therefore it will be only sketched. We will also try to use as much as possible the same notations as in Section 4.

First we present two statements that are analogous to Propositions 4.2 and 4.1:

Proposition 6.1. Let $\mathcal{D}$ be convex with a Lipschitz curvature function. For every $\alpha>0$, w.h.p.

$$
\mathcal{A}_{L}\left(L^{2} t\right) \subset L \mathcal{D}^{(\alpha)} \text { for every } t \geq 0
$$

(recall definition (2.8)). Moreover, for every $\alpha>0$ there exists $\varepsilon_{1}\left(\alpha, k_{\max }\right)>0$ such that w.h.p

$$
\mathcal{A}_{L}\left(L^{2} t\right) \supset L \mathcal{D}^{(-\alpha)} \text { for every } t \in\left[0, \varepsilon_{1}\right] .
$$

Proof. The proof of (6.1) is essentially identical to that of (4.3), so we give no detail. As for (6.2), given $\alpha$ it is possible to give a finite collection $\left\{\mathscr{D}_{i}\right\}_{i}$ such that:

- each $\mathscr{D}_{i}$ is an open convex subset of $\mathbb{R}^{2}$, obtained from (the interior of) the invariant shape $\mathscr{D}$ via a suitable translation and shrinking;

- $\mathscr{D}_{i} \subset \mathcal{D}$ for every $i$;

- $\cup_{i} \mathscr{D}_{i} \supset \mathcal{D}^{(-\alpha / 2)}$. 
Given $\eta>0$, thanks to Proposition 4.2 there exists $\varepsilon>0$ such that, w.h.p., for every $t<\varepsilon$ one has

$$
\mathcal{A}_{L}\left(L^{2} t\right) \supset \cup_{i}\left(L \mathscr{D}_{i}^{(-\eta)}\right)
$$

Here we use monotonicity (because $\mathscr{D}_{i} \subset \mathcal{D}$ ) and the fact that the union of a finite number of events which occur w.h.p. still has probability tending to 1 . Note that the choice of $\varepsilon$ is depending on $\eta$ but also on the diameter of the smallest set in the collection $\left\{\mathscr{D}_{i}\right\}_{i}$ and consequently on $k_{\text {max }}$. Then, if $\eta$ is small enough (depending on $\alpha$ ) it is clear that $\cup_{i} \mathscr{D}_{i}^{(-\eta)} \supset \mathcal{D}^{(-\alpha)}$ (recall that the $\mathscr{D}_{i}$ are open sets, so that every $x \in \mathcal{D}^{(-\alpha)}$ is contained in the interior of at least one $\left.\mathscr{D}_{i}\right)$.

Proposition 6.2. For all $\delta>0$ there exists $\varepsilon_{0}\left(\delta, k_{\min }, k_{\max }\right)>0$ such that for all $0<\varepsilon<\varepsilon_{0}$, w.h.p.,

$$
\mathcal{A}_{L}\left(L^{2} \varepsilon\right) \subset L \mathcal{D}(\varepsilon(1-\delta))
$$

and

$$
\mathcal{A}_{L}\left(L^{2} \varepsilon\right) \supset L \mathcal{D}(\varepsilon(1+\delta))
$$

where we recall that $\mathcal{D}(t)$ is the set enclosed by the curve $\gamma(t)$.

Proof of Theorem 2.2 assuming Propositions 6.2 and 6.1. It is enough to prove (2.9) for $t<$ $(1-b) t_{f}$ for arbitrary $b>0$. Then, the statement for $t \geq(1-b) t_{f}$ and also (2.11) follows from the fact that the disappearence time of a droplet of diameter $\ell$ is w.h.p. $O\left(\ell^{2}\right)$ (recall that $\gamma(t)$ shrinks to a point $t \rightarrow t_{f}$ in the sense that its diameter converges to zero). Define $k_{\min }^{*}>0$ (resp. $\left.k_{\max }^{*}<\infty\right)$ to be the infimum (resp. maximum) of $k_{\min }(s)\left(\operatorname{resp} . k_{\max }(s)\right)$ on $\left[0,(1-b) t_{f}\right]$. Fix $\delta^{\prime}$ small and let $\varepsilon<\varepsilon_{0}\left(\delta^{\prime}, k_{\min }^{*}, k_{\max }^{*}\right)$ and $\varepsilon<\varepsilon_{1}\left(\delta / 2, k_{\max }^{*}\right)$ with $\varepsilon_{0}, \varepsilon_{1}$ defined in Propositions 6.1 and 6.2. Using the Markov property and the monotonicity of our process we get that, w.h.p., for any $k$ such that $\varepsilon k<(1-b) t_{f}$

$$
\mathcal{A}_{L}\left(L^{2} k \varepsilon\right) \subset L \mathcal{D}\left(k \varepsilon\left(1-\delta^{\prime}\right)\right)
$$

From (6.5) and Proposition 6.1 we get that w.h.p., for every $t \leqslant(1-b) t_{f}$,

$$
\mathcal{A}_{L}\left(L^{2} t\right) \subset L\left[\mathcal{D}\left(\left\lfloor\frac{t}{\varepsilon}\right\rfloor \varepsilon\left(1-\delta^{\prime}\right)\right)\right]^{(\delta / 2)} \subset L\left[\mathcal{D}\left((t-\varepsilon)\left(1-\delta^{\prime}\right)\right)\right]^{(\delta / 2)} .
$$

Setting $\varepsilon^{\prime}=t_{f} \delta^{\prime}+\varepsilon$ this implies that w.h.p.

$$
\mathcal{A}_{L}\left(L^{2} t\right) \subset L \mathcal{D}\left(t-\varepsilon^{\prime}\right)^{(\delta / 2)} \quad \text { for every } \quad t \leqslant(1-b) t_{f} .
$$

Finally observe (this follows from (2.7)) that the Hausdorff distance between $\mathcal{D}\left(t-\varepsilon^{\prime}\right)$ and $\mathcal{D}(t)$ is at most $\varepsilon^{\prime} k_{\max }^{*} \max _{\theta}|a(\theta)|$ so that if $\varepsilon^{\prime}$ is chosen such that

$$
\varepsilon^{\prime} k_{\max }^{*} \max _{\theta}|a(\theta)|<\delta / 2 \text {. }
$$

we get $(2.9)$.

The lower bound is proven similarly and this is where one has to use the assumption $\varepsilon<$ $\varepsilon_{1}\left(\delta / 2, k_{\max }^{*}\right)$.

\subsection{Upper bound: Proof of (6.3).}

Definition 6.3. Define $\left(P_{i}(t)\right)_{i=1}^{4}$ to be the four "poles" of $\mathcal{D}(t)$, where the tangent vector is either horizontal or vertical (recall that $\mathcal{D}(t)$ is strictly convex at all times under our assumptions, cf. discussion after (5.12), so that the four poles are distinct and uniquely defined). $P_{1}(t)$ denotes the "north pole" and the others are numbered in the clockwise order. Denote by $\left(x\left(P_{i}(t)\right), y\left(P_{i}(t)\right)\right)$ (resp. $\left.\left(u\left(P_{i}(t)\right), v\left(P_{i}(t)\right)\right)\right)$ the coordinates of $P_{i}(t)$ in the coordinate system $\left(\mathbf{f}_{1}, \mathbf{f}_{2}\right)$ (resp. $\left(\mathbf{e}_{1}, \mathbf{e}_{2}\right)$ ). When $t=0$ we omit the time argument. 


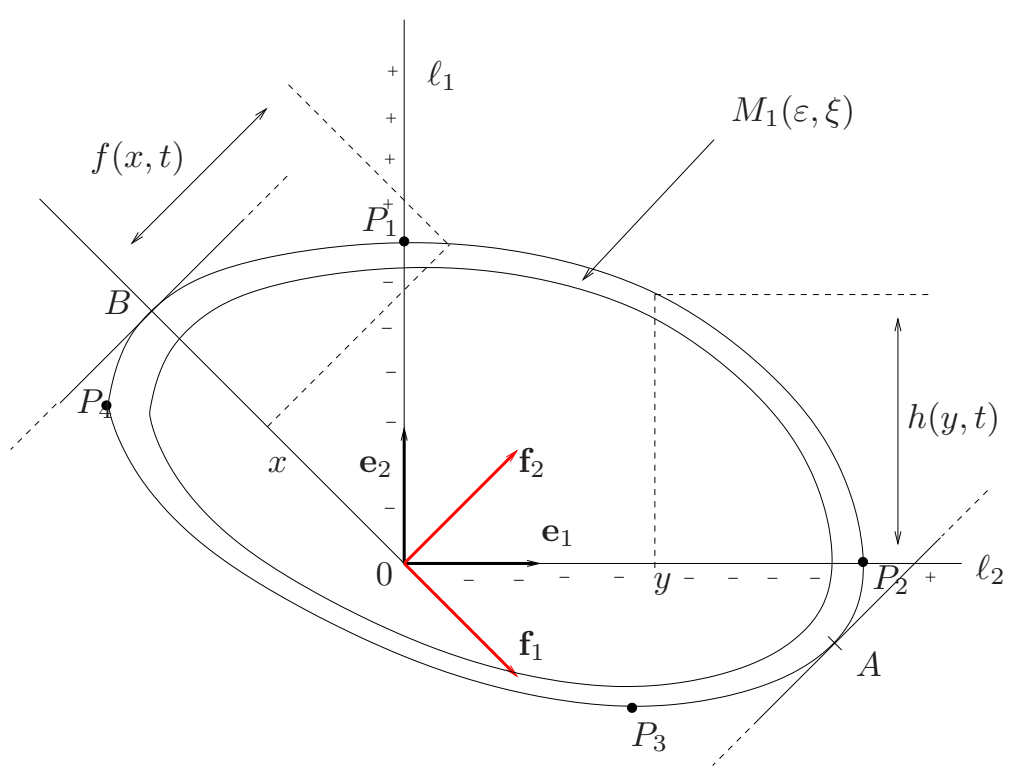

Figure 12. The larger convex set is $\mathcal{D}$ and the smaller one is $\mathcal{D}(\varepsilon(1-\delta))$. The poles $P_{i}$ of $\mathcal{D}$ are marked with black dots (for convenience we have chosen $P_{1}$ one the vertical axis and $P_{2}$ on the horizontal one). The graph in $\left(\mathbf{f}_{1}, \mathbf{f}_{2}\right)$ of the anticlockwise portion of $\partial \mathcal{D}$ between $A$ and $B$ is $f(\cdot, 0)$ and the graph in $\left(\mathbf{e}_{1}, \mathbf{e}_{2}\right)$ of the portion of $\partial \mathcal{D}$ between $P_{4}$ and $P_{2}$ is $h(\cdot, 0)$. For the proof of $(6.11)$, boundary spins to the left of $\ell_{1}$ are set to "-" below $P_{1}$ and "+" above; boundary spins below $\ell_{2}$ are set to "-" to the left of $P_{2}$ and "+" to the right.

An equivalent formulation of (6.3) is: for all $\delta>0$ and $\varepsilon$ small enough w.h.p.

$$
\sigma_{x}\left(\varepsilon L^{2}\right)=+ \text { for every } x \in L[\mathcal{D}(\varepsilon(1-\delta))]^{c} .
$$

Given some small $\xi$ we divide $[\mathcal{D}(\varepsilon(1-\delta))]^{c}$ in eight pieces $\left(M_{i}\right)_{i=1}^{4}$ and $\left(N_{i}\right)_{i=1}^{4}$ as follows (this is analogous to the definition (4.16) in the scale-invariant case, cf. Figure 6):

$$
M_{1}(\varepsilon, \xi):=\left(\left[u\left(P_{1}\right)+\xi, \infty\right) \times\left[v\left(P_{2}\right)+\xi, \infty\right)\right) \backslash \mathcal{D}(\varepsilon(1-\delta))
$$

while $N_{1}(\varepsilon, \xi)$ is the infinite component of $\left(\left[u\left(P_{1}\right)-\xi, u\left(P_{1}\right)+\xi\right] \times \mathbb{R}\right) \backslash \mathcal{D}(\varepsilon(1-\delta))$ which contains $P_{1}$. The sets $M_{i}, N_{i}$ are defined analogously for $i=2,3,4$, so that $[\mathcal{D}(\varepsilon(1-\delta))]^{c}=\bigcup_{i=1}^{4}\left(M_{i} \cup N_{i}\right)$. Equation (4.15) is proved if one can prove that for every $i$, and $\varepsilon$ small enough, w.h.p.

$$
\begin{aligned}
& \sigma_{x}\left(\varepsilon L^{2}\right)=+ \text { for every } x \in L M_{i}(\varepsilon, \xi) \\
& \sigma_{x}\left(\varepsilon L^{2}\right)=+ \text { for every } x \in L N_{i}(\varepsilon, \xi) .
\end{aligned}
$$

Of course one can focus on $i=1$, the other cases being obtained by a permutation of coordinates.

6.1.1. Proof of (6.11). We use the notation $f(\cdot, t)$ for the function whose graph in the coordinate system $\left(\mathbf{f}_{1}, \mathbf{f}_{2}\right)$ is the portion of $\partial \mathcal{D}(t)$ which goes in the anti-clockwise direction from point $A$ where the tangent forms an angle $\pi / 4$ with the horizontal axis (cf. Figure 12) to the point $B$ where the angle is $(5 / 4) \pi$. The domain of definition of $f(\cdot, t)$ decreases with time (because $\mathcal{D}(t)$ shrinks) but for $t$ small enough it includes $\left[x\left(P_{1}\right), x\left(P_{2}\right)\right]$. Let $\mathcal{D}_{1}$ be the "triangular-shaped" region delimited by $\partial \mathcal{D}$, by the vertical line $\ell_{1}$ passing through $P_{1}$ and by the horizontal line $\ell_{2}$ passing through $P_{2}$ (note that $\mathcal{D}_{1}$ may not be included in $\mathcal{D}$ ). 
We consider a modified dynamics in the north-east quadrant $\left[L u\left(P_{1}\right), \infty\right) \times\left[L v\left(P_{2}\right), \infty\right)$ delimited by the lines $L \ell_{1}, L \ell_{2}$. All the spins are initially "-" in $L \mathcal{D}_{1}$ and "+" otherwise. As for boundary spins, the spins at distance at most 1 to the left of $L \ell_{1}$ are frozen to "-" if they are below $L P_{1}$ and to "+" if they are above. The spins at distance at most 1 below $L \ell_{2}$ are frozen to "-" if they are to the left of $L P_{2}$ and to "+" otherwise, see Figure 12. In the quadrant under consideration, this dynamics dominates the original one (for the inclusion order of the set of "-" spins). Let $F_{L}(\cdot, t)$ denote the function whose graph in $\left(\mathbf{f}_{\mathbf{1}}, \mathbf{f}_{\mathbf{2}}\right)$ is the interface between "_" and "+" spins for this dynamics. Using exactly the same argument as in (4.22) we get that

$$
\lim _{L \rightarrow \infty} \sup _{x \in\left[x\left(P_{1}\right), x\left(P_{2}\right)\right]} \sup _{t \leqslant T}\left|\frac{1}{L} F_{L}\left(x L, t L^{2}\right)-g(x, t)\right|=0
$$

where $g$ is the solution for $t \geqslant 0$ and $x \in\left(x\left(P_{1}\right), x\left(P_{2}\right)\right)$ of

$$
\left\{\begin{array}{l}
\partial_{t} g(x, t)=\frac{1}{4} \partial_{x}^{2} g(x, t) \\
g(\cdot, t)=f(\cdot, 0) \\
g\left(x\left(P_{1}\right), t\right)=y\left(P_{1}\right) \text { and } g\left(x\left(P_{2}\right), t\right)=y\left(P_{2}\right) .
\end{array}\right.
$$

We are thus reduced to prove that for every $\tilde{x}_{1}, \tilde{x}_{2}$ satisfying $x\left(P_{1}\right)<\tilde{x}_{1}<\tilde{x}_{2}<x\left(P_{2}\right)$ and every $x \in\left(\tilde{x_{1}}, \tilde{x_{2}}\right)$

$$
g(x, \varepsilon)<f(x,(1-\delta) \varepsilon) .
$$

Lemma 4.3 (which is valid also in this case) allows us to write that for any fixed $\eta$, for $\varepsilon$ small enough,

$$
g(x, \varepsilon) \leqslant f(x, 0)+\frac{\varepsilon}{4}\left(\partial_{x}^{2} f(x, 0)+\eta\right) .
$$

We are left to estimate the right-hand side of (6.15). For any $\theta \in(0, \pi / 2)$ and $s>0$ define $x(\theta, s)$ to be the $\mathbf{f}_{1}$ coordinate, in the $\left(\mathbf{f}_{1}, \mathbf{f}_{2}\right)$ coordinate system, of the point of $\gamma(s)$ where the outward normal vector forms an anticlockwise angle $\theta$ with the horizontal vector $\mathbf{e}_{1}$. Note that for $s \geqslant 0 x(\cdot, s)$ defines a bijective function. We denote $\theta(\cdot, s)$ its inverse.

It is more practical for the purposes of this section to rewrite the curve-shortening flow in the $\left(\mathbf{f}_{1}, \mathbf{f}_{2}\right)$ coordinate system. Using the explicit expression (2.4) of $a(\theta)$, some trigonometry and the expression $\left|f^{\prime \prime}(x)\right| /\left(1+\left(f^{\prime}(x)\right)^{2}\right)^{3 / 2}$ for the absolute value of the curvature at the point $(x, f(x))$ of the curve given by the graph of a function $x \mapsto f(x)$, one gets that for $\theta \in(0, \pi / 2)$

$$
a(\theta) k(\theta, s)=-\frac{1}{4} \partial_{x}^{2} f(x(\theta, s), s) \cos \left(\theta-\frac{\pi}{4}\right)
$$

and

$$
\partial_{t} f(x, s)=-\frac{a(\theta(x, s)) k(\theta(x, s), s)}{\cos \left(\theta(x, s)-\frac{\pi}{4}\right)}=\frac{1}{4} \partial_{x}^{2} f(x, s),
$$

so that

$$
f(x,(1-\delta) \varepsilon)=f(x, 0)+\int_{0}^{(1-\delta) \varepsilon} \frac{1}{4} \partial_{x}^{2} f(x, s) \mathrm{d} s .
$$

We need therefore to prove time-regularity of $\partial_{x}^{2} f(\cdot, s)$ :

Lemma 6.4. For every $x\left(P_{1}\right)<\tilde{x}_{1} \leqslant \tilde{x}_{2}<x\left(P_{2}\right)$

$$
\lim _{t \rightarrow 0} \sup \left\{\left|\partial_{t} f(x, s)-\partial_{t} f(x, 0)\right|, s \in[0, t] \text { and } x \in\left[\tilde{x}_{1}, \tilde{x}_{2}\right]\right\}=0 \text {. }
$$


Proof of Lemma 6.4. Recall from Section 5 that the curvature function $k(\theta, s)$ is jointly continuous in $(\theta, s)$. Thus using equation (6.18) it is sufficient to prove that $\theta(x, s)$ is a continous function in $s$ uniformly in $x$ :

$$
\lim _{t \rightarrow 0} \sup \left\{|\theta(x, s)-\theta(x, 0)|, s \in[0, t] \text { and } x \in\left[\tilde{x}_{1}, \tilde{x}_{2}\right]\right\}=0 .
$$

This comes from the continuity of $x(\theta, \cdot)$

$$
\lim _{t \rightarrow 0} \sup \left\{|x(\theta, s)-x(\theta, 0)|, s \in[0, t] \text { and } \theta \in\left[0, \frac{\pi}{2}\right]\right\}=0
$$

and from the fact that $x(\cdot, s)$ is strictly monotone: for $t \geqslant 0$,

$$
\inf \left\{\left|\partial_{\theta} x(\theta, s)\right|, s \leqslant t, \theta \in\left[0, \frac{\pi}{2}\right]\right\}>0 .
$$

Both properties are a consequence of

$$
x(\theta, t)=x(\pi / 4, t)-\int_{\pi / 4}^{\theta} \frac{\cos \left(\theta^{\prime}-\pi / 4\right) \mathrm{d} \theta^{\prime}}{k\left(\theta^{\prime}, t\right)}
$$

which is easily derived from (5.3)-(5.4).

We finally get that for $x \in\left(\tilde{x}_{1}, \tilde{x}_{2}\right)$ and $\varepsilon$ small enough,

$$
f(x,(1-\delta) \varepsilon) \geq f(x, 0)+(1-\delta) \frac{\varepsilon}{4}\left(\partial_{x}^{2} f(x, 0)-\eta\right) .
$$

Thus, combining this with $(6.16),(6.15)$ is proved if one has

$$
\partial_{x}^{2} f(x, 0)+\eta \leqslant(1-\delta)\left(\partial_{x}^{2} f(x, 0)-\eta\right)
$$

i.e.

$$
2 \eta+\delta \partial_{x}^{2} f(x, 0) \leqslant 0 .
$$

For this it is sufficient to have $\eta$ small enough, since (cf. (6.18)) $\sup \left\{\partial_{x}^{2} f(x, 0), x \in\left[x\left(P_{1}\right), x\left(P_{2}\right)\right]\right\}$ can be upper bounded by a negative constant times the minimal curvature $k_{\min }$, which is strictly positive.

6.1.2. Proof of (6.12). Set $h(\cdot, t)$ to be the continuous concave function whose graph in the $\left(\mathbf{e}_{1}, \mathbf{e}_{2}\right)$ coordinate system is the portion of of $\gamma(t)$ which goes from $P_{2}(t)$ to $P_{4}(t)$ with the anticlockwise orientation. Given a small $\eta$ choose $\xi$ small enough so that $\sup \left\{\left|\partial_{x} h(x, 0)\right|, u\left(P_{1}\right)-\right.$ $\left.\xi \leqslant x \leqslant u\left(P_{1}\right)+\xi\right\} \leq \eta$.

Consider $\bar{h}(\cdot)$ the $C^{1}$ function equal to $h(\cdot, 0)$ on $\left[u\left(P_{1}\right)-2 \xi, u\left(P_{1}\right)+2 \xi\right]$ and linear outside. Assume for definiteness that $\bar{h}\left(u\left(P_{1}\right)-4 \xi\right) \leq \bar{h}\left(u\left(P_{1}\right)+4 \xi\right)$. Define $\xi^{-}=u\left(P_{1}\right)-4 \xi$ and $\xi^{+}=\inf \left\{x>u\left(P_{1}\right), \bar{h}(x)=\bar{h}\left(\xi^{-}\right)\right\}$. We consider the restriction of $\bar{h}$ to $\left[\xi^{-}, \xi^{+}\right]$and still call it $\bar{h}$. Define

$$
J^{1}:=\left[\xi^{+}, \infty\right) \times\left[\bar{h}\left(\xi^{+}\right), \infty\right), \quad J^{2}:=\left(-\infty, \xi^{-}\right] \times\left[\bar{h}\left(\xi^{+}\right), \infty\right) .
$$

We consider the same chain of monotonicities as in the scale-invariant case (Section 4.3.2) and we end up with a dynamics in the half-strip $\left[L \xi^{-}, L \xi^{+}\right] \times\left[L \bar{h}\left(\xi^{+}\right), \infty\right)$ with boundary spins frozen to "+" in $L\left(J^{1} \cup J^{2}\right)$ and to "-" in $\mathbb{Z}^{*} \times\left(-\infty, L \bar{h}\left(\xi^{+}\right)\right]$and an initial condition with "-" spins under the graph of $L \bar{h}(\cdot / L)$. Also, the dynamics thus obtained does not allow moves that make the interface non-connected. Calling $\left(\sigma_{2}(t)\right)_{t \geqslant 0}$ this dynamics, (4.35) is satisfied.

Define $H_{L}:\left[L \xi^{-}, L \xi^{+}\right] \rightarrow \mathbb{Z}$ to be the function whose graph in $\left(\mathbf{e}_{1}, \mathbf{e}_{2}\right)$ is the interface between "+" and "-" spins. We have to prove

$$
\frac{1}{L} H_{L}\left(L x, \varepsilon L^{2}\right) \leq h(x,(1-\delta) \varepsilon) \text { for every } x \in\left(u\left(P_{1}\right)-\xi, u\left(P_{1}\right)+\xi\right) \text {. }
$$


Following the same steps as in (4.37) to (4.40) one finds that the left-hand side of (6.29) is upper bounded w.h.p. by

$$
h(x, 0)+\frac{\varepsilon}{2}(1+\eta)^{-2}\left(\partial_{x}^{2} h\left(u\left(P_{1}\right), 0\right)+r(x)\right)+o(\varepsilon),
$$

where $r(x)$ tends to 0 when $x \rightarrow u\left(P_{1}\right)$.

To estimate the r.h.s of (6.29), one remarks that, in analogy with (6.18),

$$
\partial_{s} h(x, s)=-a(\theta(x, s)) k(\theta(x, s), s) / \sin (\theta(x, s))
$$

so that $\partial_{t} h(x, t)$ is continuous in $x$ and $t$ ( $\operatorname{since} \theta$ is around $\pi / 2, \sin (\theta(x, s))$ is bounded away from zero). Moreover

$$
\partial_{t} h\left(u\left(P_{1}\right), 0\right)=\frac{1}{2} \partial_{x}^{2} h\left(u\left(P_{1}\right), 0\right),
$$

which can be obtained directly from $a(0)=1 / 2$ and from the fact that the curvature of $\mathcal{D}$ at the north pole $P_{1}$ equals minus the second derivative of $h(x, 0)$ computed at $x=u\left(P_{1}\right)$. Thus

$$
h(x,(1-\delta) \varepsilon) \geqslant h(x, 0)+(1-\delta) \frac{\varepsilon}{2}(1+\eta) \partial_{x}^{2} h\left(u_{1}, 0\right),
$$

and (6.29) is proven (combining (6.30) and (6.33)) choosing $\eta$ and $\xi$ small enough.

6.2. Lower bound: Proof of (6.4). We are confident that the reader is by now convinced that the proof of Theorem 2.2 is essentially identical to that in the scale-invariant case, modulo the fact that the definitions of the various subsets of $\mathbb{R}^{2}$ needed to define the regions where spins are frozen to "-" or "+" $\left(U, J^{1}, J^{2}\right.$, etc) have to be adapted in the obvious way due to the lack of discrete-rotation symmetry of the general initial droplet $\mathcal{D}$. We will therefore skip altogether the proof of (6.4) and we limit ourselves to indicating the only point where some (minor) care has to be taken.

The definition (4.45) of the set $U$ is replaced by $U:=\mathcal{D}^{(-\nu)}$, cf. (2.8). Let $s_{1}$ be the vertical segment obtained moving downwards from the "north pole of $U$ " until the point $c$ where $s_{1}$ meets $s_{2}$, the horizontal segment obtained moving to the left from the "east pole" of $U$ until $c$ is reached. To prove the analog of (4.48), mimicking the proof given in Section 4.4.4, one would like to apply (6.2) in order to freeze to "-" all the spins along the two rescaled segments $L s_{1}, L s_{2}$. This is however not allowed in general, because nothing guarantees that they are entirely contained in $L U$, i.e., that $c \in U$ (this problem does not occur for the invariant shape $\mathscr{D}$, where $c$ is the origin). The solution however is simple: one just freezes to "-" all the spins along the portions of $L s_{1}, L s_{2}$ which are inside $L U$, and along the shorter portion of $L \partial U$ which connects them (call $\Gamma$ this portion). The point is that in this situation the $+/-$ interface between north and east poles follows again the corner dynamics and Theorem 3.2 is applicable. The freezing of "-" spins along $\Gamma$ is equivalent to putting a hard-wall constraint in the corner dynamics (the interface is not allowed to cross a zig-zag path which approximates $\Gamma$ ) but this is irrelevant: since $\Gamma$ is at distance of order $L$ away from the linear profile the corner dynamics approaches for long times, the probability that the interface even feels the hard-wall constraint within the diffusive times of order $L^{2}$ we are interested in goes to zero with $L$ (this again can be seen via Theorem 3.2). Other than that, the proof of (6.4) is identical to that in the $\mathcal{D}=\mathscr{D}$ case. 


\section{Proof of Theorem 3.2: ScAling Limit For SSEP}

The first step is to discretize (3.6), so that instead of working with $\phi(\cdot, \cdot)$ we get $\Phi(\cdot, \cdot)$ solution of the analogous discrete Cauchy problem:

$$
\left\{\begin{array}{l}
\partial_{t} \Phi(x, t)=\frac{1}{2} \Delta \Phi(x, t) \\
\Phi^{L}(0, t)=h_{0}^{0}=0 \\
\Phi^{L}(L, t)=h_{L}^{0} \\
\Phi^{L}(x, 0)=h_{x}^{0}
\end{array}\right.
$$

for every $t \geq 0$ and $x \in\{1, \ldots, L-1\}$. Here $\Delta$ is the discrete Laplacian operator:

$$
(\Delta f)(x):=f(x+1)+f(x-1)-2 f(x) \quad \forall x \in\{1, \ldots, L-1\} .
$$

Note that $\Phi(x, t)=\mathbb{E}\left[h_{x}(t)\right]$, with $(h(t))_{t \geq 0}$ the process with generator (3.3), and that $\Phi(0, t)-$ $h_{0}(t)=\Phi(L, t)-h_{L}(t)=0$. It is a standard result that $\Phi$, solution of the discrete space heatequation, converges to $\phi$ in all reasonable norms when $L \rightarrow \infty$ in the diffusive limit. We record this result here:

\section{Lemma 7.1.}

$$
\lim _{L \rightarrow \infty} \max _{t \in[0, T]} \max _{x \in[0,1]} \frac{1}{L}\left|\Phi\left(\lfloor x L\rfloor, t L^{2}\right)-L \phi(x, t)\right|=0
$$

Using Lemma 7.1, we are reduced to prove

$$
\lim _{L \rightarrow \infty} \mathbb{P}\left[\max _{t \in\left[0, T L^{2}\right]} \max _{x \in\{1, \ldots, L-1\}}\left|h_{x}(t)-\Phi(x, t)\right|<\varepsilon L\right]=1 .
$$

Both $h .(t)$ and $\Phi(\cdot, t)$ are 1-Lipschitz functions (for all $t$ ) so that $|h .(t)-\Phi(\cdot, t)|$ is 2-Lipschitz and

$$
\left\{\max _{x \in\{1, \ldots, L-1\}}\left|h_{x}(t)-\Phi(x, t)\right| \geqslant a\right\} \Longrightarrow\left\{\sum_{x=1}^{L-1}\left[h_{x}(t)-\Phi(x, t)\right]^{2} \geqslant a^{3} / 3\right\} .
$$

As a consequence, (7.4) is equivalent to prove the following $\mathbb{L}_{2}$ convergence statement:

Proposition 7.2. The following convergence in probability holds:

$$
\lim _{L \rightarrow \infty} \sup _{t \in\left[0, L^{2} T\right]} \frac{1}{L^{3}} \sum_{x=1}^{L-1}\left[h_{x}(t)-\Phi(x, t)\right]^{2}=0 .
$$

Proof of Proposition 7.2. The restriction of the operator $\Delta$ to

$$
\Lambda_{L}=\{g:\{0, \ldots, L\} \mapsto \mathbb{R}, g(0)=g(L)=0\}
$$

is self-adjoint (for the canonical scalar product on $\mathbb{R}^{L-1}$ denoted in the sequel by $\langle\cdot, \cdot\rangle$ ) and the family of functions

$$
f_{k}:\{0, \ldots, L\} \ni x \mapsto \sqrt{\frac{2}{L}} \sin \left(\frac{k \pi x}{L}\right), \quad k=1, \ldots, L-1
$$

forms an orthonormal basis of $\Lambda_{L}$ of $\Delta$-eigenfunctions, with respective eigenvalues

$$
-\lambda_{k}:=2 \cos \left(\frac{\pi k}{L}\right)-2<0 .
$$


As the function $x \mapsto h_{x}(t)-\Phi(x, t)$ is in $\Lambda_{L}$ it can be decomposed on this basis. We use the notation $H_{t}^{k}$ for its $k$-th coordinate (multiplied by $\sqrt{L / 2}$ for convenience):

$$
H_{t}^{k}:=\sum_{x=0}^{L}\left[h_{x}(t)-\Phi(x, t)\right] \sin \left(\frac{k \pi x}{L}\right) .
$$

The quantity one wants to estimate in (7.6) is equal to

$$
\sup _{t \in\left[0, L^{2} T\right]} \frac{2}{L^{4}} \sum_{k=1}^{L-1}\left(H_{t}^{k}\right)^{2} \leq \frac{2}{L^{4}} \sum_{k=1}^{L-1} \sup _{t \in\left[0, L^{2} T\right]}\left(H_{t}^{k}\right)^{2} .
$$

We control the right-hand side by controlling each $H_{t}^{k}$ separately.

Lemma 7.3. For every $L, k \in\{1, \ldots, L-1\}$ and $t>0$ one has deterministically

$$
\left|H_{t}^{k}\right| \leq 4 L^{2} / k \text {. }
$$

Moreover for any given T, w.h.p.

$$
\left|H_{t}^{k}\right| \leq L^{7 / 4} \quad \text { for every } \quad k \leq(\log L)^{1 / 3} \quad \text { and every } t \leq L^{2} T .
$$

Proof of Lemma 7.3. The first point is easy. Using summation by parts

$$
H_{t}^{k}=\sum_{x=1}^{L}\left(\left[h_{x}(t)-\Phi(x, t)\right]-\left[h_{x-1}(t)-\Phi(x-1, t)\right]\right) \sum_{y=x}^{L} \sin \left(\frac{k \pi y}{L}\right) .
$$

Then one can check that for every $x$ and $k$

$$
\begin{gathered}
\left|\left[h_{x}(t)-\Phi(x, t)\right]-\left[h_{x-1}(t)-\Phi(x-1, t)\right]\right| \leq 2, \\
\left|\sum_{y=x}^{L} \sin \left(\frac{k \pi y}{L}\right)\right| \leq \frac{2 L}{k}
\end{gathered}
$$

so that (7.11) follows.

For the second point, first, one notices that for all $k \in\{1, \ldots, L-1\}$, the functions

$$
F_{k}: \Omega_{M_{L}, N_{L}} \ni h \mapsto \sum_{x=0}^{L} \sin \left(\frac{\pi k x}{L}\right)\left[h_{x}-\frac{h_{L}-h_{0}}{L} x\right]
$$

are eigenfunctions of $\mathcal{L}$ with respective eigenvalues $-\lambda_{k}$. Indeed $F_{k}$ is just a linear combination of the coordinate function $A_{x}: h \mapsto h_{x}$ (plus a constant), and it can be seen from the very definition of the generator $\mathcal{L}(3.3)$

$$
\mathcal{L}\left(A_{x}\right)(h)=\frac{1}{2}(\Delta h)(x)=\frac{1}{2}(\Delta \tilde{h})(x)
$$

using the notation $\tilde{h}_{x}=h_{x}-\frac{h_{L}-h_{0}}{L} x$ so that (note that $\tilde{h} \in \Lambda_{L}$ )

$$
2 \mathcal{L} F_{k}(h)=\sqrt{L / 2}\left\langle f_{k}, \Delta \tilde{h}\right\rangle=\sqrt{L / 2}\left\langle\Delta f_{k}, \tilde{h}\right\rangle=-\lambda_{k} \sqrt{L / 2}\left\langle f_{k}, \tilde{h}\right\rangle=-\lambda_{k} F_{k}(h) .
$$

As a consequence one can rewrite

$$
H_{t}^{k}=\sum_{x=0}^{L} \sin \left(\frac{k \pi x}{L}\right) \tilde{h}_{x}(t)-e^{-\lambda_{k} t / 2} \sum_{x=0}^{L} \sin \left(\frac{k \pi x}{L}\right) \tilde{h}_{x}(0)
$$

and notice that $M_{t}^{k}:=e^{\lambda_{k} t / 2} H_{t}^{k}$ is a martingale. Therefore one can get the result by computing the second moment of $M_{t}^{k}$ and using Doob's inequality. 
It is not difficult to bound the quadratic variation of $M^{k}$. Notice that

$$
\mathbb{E}\left[\left(M_{t}^{k}\right)^{2}\right]=\mathbb{E}\left[\int_{0}^{t} \mathrm{~d}\left\langle M^{k}\right\rangle_{s}\right]
$$

and that

$$
\mathrm{d}\left\langle M^{k}\right\rangle_{s}=e^{\lambda_{k} s} \mathrm{~d}\left\langle H^{k}\right\rangle_{s}=e^{\lambda_{k} s} \sum_{k=1}^{L-1} \sin ^{2}\left(\frac{k \pi x}{L}\right) \frac{(\Delta(h(t))(x))^{2}}{4} \mathrm{~d} s \leq L e^{\lambda_{k} s} \mathrm{~d} s
$$

so that $\mathbb{E}\left[\left(M_{t}^{k}\right)^{2}\right] \leq L \int_{0}^{t} e^{\lambda_{k} s} \mathrm{~d} s$. Therefore (using $\lambda_{k}=\pi^{2} k^{2} / L^{2}(1+o(1)$ ) uniformly for all $\left.k \leqslant(\log L)^{1 / 3}\right)$,

$$
\mathbb{P}\left[\sup _{t \in\left[0, L^{2} T\right]}\left|H_{t}^{k}\right| \geq a\right] \leq \mathbb{P}\left[\sup _{t \in\left[0, L^{2} T\right]}\left|M_{t}^{k}\right| \geq a\right] \leq C \frac{L^{3} e^{\lambda_{k} L^{2} T}}{a^{2} k^{2}} .
$$

Using this inequality for $a:=L^{7 / 4}$ and all $k \leqslant(\log L)^{1 / 3}$ one gets that

$$
\mathbb{P}\left[\exists t \in\left[0, L^{2} T\right], \exists k \leqslant(\log L)^{1 / 3},\left|H_{t}^{k}\right| \geq L^{7 / 4}\right] \leq \sum_{k \leqslant(\log L)^{1 / 3}} \frac{C}{k^{2} \sqrt{L}} e^{k^{2} \pi^{2} T} .
$$

One can check that the right-hand side above tends to zero when $L$ goes to infinity, which finishes the proof of Lemma 7.3.

We now turn to $(7.10)$ :

$$
\frac{2}{L^{4}} \sum_{k=1}^{L-1} \sup _{t \in\left[0, L^{2} T\right]}\left(H_{t}^{k}\right)^{2} \leq \frac{2}{L^{4}} \sum_{k \leqslant(\log L)^{1 / 3}} \sup _{t \in\left[0, L^{2} T\right]}\left(H_{t}^{k}\right)^{2}+32 \sum_{k=\left\lceil(\log L)^{1 / 3}\right]}^{L} k^{-2} .
$$

The second term tends to zero (it is roughly $(\log L)^{-1 / 3}$ ). The first one is less than

$$
\frac{2}{L^{4}} \sum_{k \leqslant(\log L)^{1 / 3}} L^{7 / 2} \leqslant \frac{\log L}{\sqrt{L}} .
$$

This achieves the proof of Proposition 7.2 and thus also the one of Theorem 3.2.

\section{Appendix A. Proof of Theorem 3.4: scaling limit for the Zero-range process}

This section follows quite closely computations in Appendix A of [24].

A.1. Particle system and monotonicity. For $x \in\{-L, \ldots, L\}$ we denote $\eta_{x}:=h_{x+1}-h_{x}$ the discrete gradient of $h$ in $x$. A configuration $h \in \Omega_{L}$ can be alternatively given by $\eta \in \Theta_{L}:=$ $\{\eta:\{-L, \ldots, L\} \rightarrow \mathbb{Z}\}$. It turns out that the zero-range process description of the dynamics (cf. Section 3.2) is easier to work with.

For a more formal description of the dynamics we write explicitly its generator. For $\eta \in \Theta_{L}$ and $x \in\{-L, \ldots, L-1\}$, we define the configuration $\vec{\eta}^{(x)}$ as

$$
\begin{aligned}
\vec{\eta}^{(x)}(x) & :=\eta_{x}-\operatorname{sg}\left(\eta_{x}\right), \\
\vec{\eta}^{(x)}(x+1) & :=\eta_{x+1}+\operatorname{sg}\left(\eta_{x}\right), \\
\vec{\eta}^{(x)}(y) & :=\eta_{y}, \quad \forall y \notin\{x, x+1\} .
\end{aligned}
$$


We define $\overleftarrow{\eta}^{(x)}$ analogously for $x \in\{-L+1, \ldots, L\}$ replacing $x+1$ in the second and third lines by $x-1$. The sign function sg is given by

$$
\operatorname{sg}(a):=\left\{\begin{array}{l}
1 \text { if } a>0, \\
-1 \text { if } a<0, \\
0 \text { if } a=0 .
\end{array}\right.
$$

The generator of the chain seen in the state-space $\Theta_{L}$ is given by

$$
\mathcal{L} f:=\frac{1}{2} \sum_{x=-L}^{L-1}\left[f\left(\vec{\eta}^{(x)}\right)+f\left(\overleftarrow{\eta}^{(x+1)}\right)-2 f(\eta)\right]
$$

Note that the dynamics conserves the sum of the $\eta$ 's, i.e. the value of $h_{L+1}$.

Before going to the core of the proof, we need to change slightly the initial condition. In order to compare with the original one, one needs the following monotonicity statement:

Proposition A.1. [Coupling]

(i) There is a canonical way of constructing simultaneously the dynamics with generator (3.8) from all possible initial configurations $h^{0}$. It satisfies the following monotonicity property: given $h^{0}$ and $\bar{h}^{0}$ with $h_{x}^{0} \geq \bar{h}_{x}^{0}$ for all $x$, the dynamics $h$ and $\bar{h}$ starting from $h^{0}$ and $\bar{h}^{0}$ respectively satisfy $h_{x}(t) \geq \bar{h}_{x}(t)$ for every $t$ and $x$. Moreover, the dynamics started from $h^{0}+a, a \in \mathbb{Z}$, (a vertically translated version of $h^{0}$, including the boundary conditions $h_{0}$ and $\left.h_{L+1}\right)$, is simply $(h(t)+a)_{t \geq 0}$.

(ii) There is a canonical way of constructing the dynamics with generator (A.3) from all possible initial configurations $\eta^{0}$. It satisfies the following monotonicity property: given $\eta^{0}$ and $\bar{\eta}^{0}$ with $\eta_{x}^{0} \geq \bar{\eta}_{x}^{0}$ for all $x$, the dynamics $\eta$ and $\bar{\eta}$ starting from $\eta^{0}$ and $\bar{\eta}^{0}$ respectively satisfy $\eta_{x}(t) \geq \bar{\eta}_{x}(t)$ for every $t$ and $x$.

Proof. The idea of the proof is using a canonical construction of the process, similarly to what is done in Section 2.3. It is quite classic but we perfom it here for the sake of completeness.

- For $x \in\{-L+1, L\}$ we define $\left(\tau_{n, x}\right)_{n \geq 0}$ and $\left(\tau_{n, x}^{\prime}\right)_{n \geq 0}$ to be two IID clock processes, with $\tau_{0, x}=0$ and $\tau_{n+1, x}-\tau_{n, x}$ IID exponential variables of mean 2 .

- The process $h(\cdot)$ is constant in time except at the of the ringing times of the clock processes. At time $\tau_{n, x}$ only $h_{x}$ is modified, as follows: $h_{x}\left(\tau_{n, x}+0^{+}\right)=h_{x}\left(\tau_{n, x}\right)+$ $\operatorname{sg}\left(h_{x-1}\left(\tau_{n, x}\right)-h_{x}\left(\tau_{n, x}\right)\right)$, the other coordinates being left unchanged. At time $\tau_{n, x}^{\prime}$ only $h_{x}$ is modified, as follows: $h_{x}\left(\tau_{n, x}^{\prime}+0^{+}\right)=h_{x}\left(\tau_{n, x}^{\prime}\right)+\operatorname{sg}\left(h_{x+1}\left(\tau_{n, x}^{\prime}\right)-h_{x}\left(\tau_{n, x}^{\prime}\right)\right)$, the other coordinates being left unchanged.

The reader can check that this allows to couple the dynamics from all possible initial conditions and that our coupling has the desired properties. This coupling induces a coupling on $\eta$ that also has the right properties.

A.2. Changing the initial condition. We prove (3.14) working with an initial condition which is not the one, $h^{0}$, described in (3.11), which is random and for which the number of particle at a site is given by a geometric variable. The reason for this change of initial condition will appear in the proof of $(\mathrm{iii})$ in Lemma A.3. We explain in this section why this implies the result starting from $h^{0}$.

Given a continuous function $\phi^{0}:[-1,1] \rightarrow \mathbb{R}$ with $\phi^{0}( \pm 1)=0$ and with a finite number of changes of monotonicity, set $\left(\hat{\eta}_{x}\right)_{x \in\{-L, \ldots, L\}}$ to be a family of independent variables with the following distribution: if $\phi^{0}((x+1) / L)-\phi^{0}(x / L) \geq 0$ then $\hat{\eta}_{x}$ is a geometric variable of mean 
$L\left(\phi^{0}((x+1) / L)-\phi^{0}(x / L)\right)$ and if $\left(\phi^{0}((x+1) / L)-\phi^{0}(x / L)\right)<0$ then $-\hat{\eta}_{x}$ is a geometric variable of mean $L\left(\phi^{0}(x / L)-\phi^{0}((x+1) / L)\right)$ (with the convention that $\phi^{0}(1+1 / L)=0$ ). One sets

$$
\hat{h}_{x}^{0}=\sum_{y=-L}^{x-1} \hat{\eta}_{y} .
$$

Note that for every $\varepsilon>0$, w.h.p,

$$
\hat{h}_{x}^{0}-L^{1 / 2+\varepsilon} \leq h_{x}^{0} \leq \hat{h}_{x}^{0}+L^{1 / 2+\varepsilon} \text { for every } \quad x \in\{-L, \ldots, L+1\} .
$$

Let $(h(t))_{t \geq 0},(\hat{h}(t))_{t>0}$ be the dynamics with generator (3.8) started with initial condition $h^{0}$, $\hat{h}^{0}$ respectively, constructed using the canonical way of Proposition A.1 (i). Then with high probability, for every $t>0$ and $x \in\{-L, \ldots, L\}$

$$
\hat{h}_{x}(t)-L^{1 / 2+\varepsilon} \leq h_{x}(t) \leq \hat{h}_{x}(t)+L^{1 / 2+\varepsilon} .
$$

Therefore in order to prove $(3.14)$ for $h(\cdot)$, it is sufficient to prove it for $\hat{h}(\cdot)$. We let $\hat{\eta}_{x}(t)=$ $\hat{h}_{x+1}(t)-\hat{h}_{x}(t)$ denote the gradients of $\hat{h}$.

A.3. Proof of an $\mathbb{L}_{2}$ statement. For $(\hat{h}(t))_{t \geq 0}$ defined above one has

Proposition A.2. For any $t \geq 0$

$$
\lim _{L \rightarrow \infty} \mathbb{E}\left[\frac{1}{L^{3}} \sum_{x=-L}^{L+1}\left(\Phi\left(x, L^{2} t\right)-\hat{h}_{x}\left(L^{2} t\right)\right)^{2}\right]=0
$$

This result does not directly imply (3.14) ( $\hat{h}$ may have a priori unbounded gradients), but it is not to difficult conclude from Proposition A.2, see Section A.4. In the rest of the section, for lightness of notation we write $h, \eta$ instead of $\hat{h}, \hat{\eta}$.

Before starting the proof we need some technical statements. First note, recalling the definition of the generator (3.8), that for every $x \in\{-L+1, \ldots, L\}$

$$
\begin{aligned}
2 \partial_{t} \mathbb{E}\left[h_{x}(t)\right] & =\mathbb{E}\left[\operatorname{sg}\left(\eta_{x}(t)\right)-\operatorname{sg}\left(\eta_{x-1}(t)\right)\right], \\
2 \partial_{t} \mathbb{E}\left[h_{x}^{2}(t)\right] & =\mathbb{E}\left[2 h_{x}(t)\left(\operatorname{sg}\left(\eta_{x}(t)\right)-\operatorname{sg}\left(\eta_{x-1}(t)\right)+\left(\left|\operatorname{sg}\left(\eta_{x}(t)\right)\right|+\left|\operatorname{sg}\left(\eta_{x-1}(t)\right)\right|\right)\right] .\right.
\end{aligned}
$$

Now some remarks:

Lemma A.3. The following holds (recall notations in (3.13)):

(i) $\max _{x}\left|q_{x}(t)\right|$ is a non-increasing function of $t$. As a consequence

$$
\forall t>0, \forall x \in\{-L, \ldots, L\},\left|q_{x}(t)\right| \leq\left\|\partial_{x} \phi^{0}\right\|_{\infty} .
$$

(ii) $\max _{x}\left|\sigma\left(q_{x+1}(t)\right)-\sigma\left(q_{x}(t)\right)\right|$ is a non-increasing function of $t$ (recall $\left.\sigma(u)=u /(1+|u|)\right)$. Then, for some $C\left(\phi^{0}\right)=C\left(\left\|\partial_{x} \phi^{0}\right\|_{\infty},\left\|\partial_{x}^{2} \phi^{0}\right\|_{\infty}\right)<\infty$

$$
\forall t>0, \forall x \in\{-L, \ldots, L\},\left|q_{x+1}(t)-q_{x}(t)\right| \leq C\left(\phi^{0}\right) / L .
$$

(iii) For any $t$, the random vectors $\left(\eta_{x}(t)\right)_{x \in\{-L, \ldots, L\}}$ and $\left(-\eta_{x}(t)\right)_{x \in\{-L, \ldots, L\}}$ are stochastically dominated by $2 L+1$ IID geometric variables with mean $\left\|\partial_{x} \phi^{0}\right\|_{\infty}$.

Proof. For $(i)$ it is sufficient to show that $Q(t)=\max _{x} q_{x}(t)$ is non-increasing (by a similar argument one shows that $\min q_{x}(t)$ is non-decreasing). As the maximum over finitely many differentiable functions, $\max _{x} q_{x}(t)$ possesses a right and a left-derivative everywhere and the right-derivative is equal to

$$
\partial_{t}^{+} Q(t)=\max _{x \in \operatorname{argmax} q \cdot(t)} \partial_{t} q_{x}(t)
$$


For any $x$ in $\max _{x \in \operatorname{argmax} q .(t)}$, one has

$$
2 \partial_{t} q_{x}(t)=\sigma\left(q_{x+1}(t)\right)+\sigma\left(q_{x-1}(t)\right)-2 \sigma\left(q_{x}(t)\right) \leq 0
$$

(as $\sigma\left(q_{x}(t)\right)$ is maximal), and therefore $Q(t)$ is decreasing.

For $(i i)$ : Using the same argument as for the point $(i)$, we just have to note that for any fixed time $T$ and $x_{0}$ where $\max _{x}\left[\sigma\left(q_{x+1}\right)-\sigma\left(q_{x}\right)\right](T)$ is attained one has

$$
\begin{aligned}
2\left[\partial_{t}\left\{\sigma\left(q_{x_{0}+1}\right)-\sigma\left(q_{x_{0}}\right)\right\}\right] & (T)=\sigma^{\prime}\left(q_{x_{0}+1}(T)\right)\left[\sigma\left(q_{x_{0}+2}(T)\right)-\sigma\left(q_{x_{0}+1}(T)\right)\right] \\
& +\sigma^{\prime}\left(q_{x_{0}}(T)\right)\left[\sigma\left(q_{x_{0}}(T)\right)-\sigma\left(q_{x_{0}-1}(T)\right)\right] \\
- & {\left[\sigma^{\prime}\left(q_{x_{0}+1}(T)\right)+\sigma^{\prime}\left(q_{x_{0}}(T)\right)\right]\left[\sigma\left(q_{x_{0}+1}(T)\right)-\sigma\left(q_{x_{0}}(T)\right)\right] \leq 0 . }
\end{aligned}
$$

For (iii): One has that $L\left(\phi^{0}\left(\frac{x+1}{L}\right)-\phi^{0}\left(\frac{x}{L}\right)\right) \leq\left\|\partial_{x} \phi^{0}\right\|_{\infty}$ so that the initial configuration $\eta^{0}$ is stochastically dominated by $\tilde{\eta}^{0}$ the configuration given by $2 L+1$ IID geometric variables with mean $\left\|\partial_{x} \phi^{0}\right\|_{\infty}$. According to Proposition A.1 (ii), one can couple the two dynamics $\eta$ and $\tilde{\eta}$ starting from $\eta^{0}$ and $\tilde{\eta}^{0}$ so that $\eta(t) \leq \tilde{\eta}(t)$ for all $t \geq 0$. For fixed $t$ the law of $\tilde{\eta}(t)$ is the same as the one of $\tilde{\eta}^{0}$ as this distribution is stationary for the dynamics. The other domination is proved in the same way.

Proof of Proposition A.2. We estimate the difference between $\mathbb{E}\left[\frac{1}{L^{3}} \sum_{x=-L}^{L+1}\left(\Phi\left(x, L^{2} t\right)-h_{x}\left(L^{2} t\right)\right)^{2}\right]$ and the same quantity at time zero, by considering it as the integral of the derivative.

$$
\begin{gathered}
\mathbb{E}\left[\frac{1}{L^{3}} \sum_{x=-L}^{L+1}\left(\Phi\left(x, L^{2} t\right)-h_{x}\left(L^{2} t\right)\right)^{2}\right]-\mathbb{E}\left[\frac{1}{L^{3}} \sum_{x=-L}^{L+1}\left(\Phi(x, 0)-h_{x}(0)\right)^{2}\right] \\
=\frac{1}{L^{3}} \int_{0}^{L^{2} t} \sum_{x=-L+1}^{L} \partial_{s} \mathbb{E}\left[\left(\Phi(x, s)-h_{x}(s)\right)^{2}\right] \mathrm{d} s \\
=\frac{1}{L^{3}} \sum_{x=-L+1}^{L} \int_{0}^{L^{2} t} \mathbb{E}\left\{\left(\Phi(x, s)-h_{x}(s)\right)\left(\sigma\left(q_{x}(s)\right)-\sigma\left(q_{x-1}(s)\right)\right)\right. \\
+h_{x}(s)\left(\operatorname{sg}\left(\eta_{x}(s)\right)-\operatorname{sg}\left(\eta_{x-1}(s)\right)+\frac{1}{2}\left(\left|\operatorname{sg}\left(\eta_{x}(s)\right)\right|+\mid \operatorname{sg}\left(\eta_{x-1}(s) \mid\right)\right\} \mathrm{d} s\right. \\
=\frac{1}{L^{3}} \sum_{x=-L}^{L} \int_{0}^{L^{2} t} \mathbb{E}\left[-q_{x}(s) \sigma\left(q_{x}(s)\right)+\eta_{x}(s) \sigma\left(q_{x}(s)\right)+q_{x}(s) \operatorname{sg}\left(\eta_{x}(s)\right)\right. \\
-\frac{1}{L^{3}} \int_{0}^{L^{2} t} \mathbb{E}\left[h_{L+1}(s)\left(\operatorname{sg}\left(\eta_{L}(s)\right)-\sigma\left(\eta_{L}(s)\right)\right)+\frac{1}{2}\left(\mid \operatorname{sg}\left(\eta_{-L}(s)|+| \operatorname{sg}\left(\eta_{L}(s) \mid\right)\right] \mathrm{d} s .\right.\right.
\end{gathered}
$$

The second equality is obtained by expanding the product and using (A.8) and (3.12) to estimate all the derivated terms. The third equality is obtained via summation by parts, it gives a term 
that is due to boundary effect (the second one) which can be bounded as follows

$$
\begin{array}{r}
L^{-3}\left|\int_{0}^{L^{2} t} \mathbb{E}\left[h_{L+1}(s)\left(\operatorname{sg}\left(\eta_{L}(s)\right)-\sigma\left(q_{L}(s)\right)\right)+\frac{1}{2}\left(\left|\operatorname{sg}\left(\eta_{-L}(s)\right)\right|+\left|\operatorname{sg}\left(\eta_{L}(s)\right)\right|\right)\right] \mathrm{d} s\right| \\
\leq C L^{-1}\left(1+\mathbb{E}\left|h_{L+1}\right|\right)=O\left(L^{-1 / 2}\right) .
\end{array}
$$

Indeed $h_{L+1}(t)$ is constant through time and is the sum of $2 L+1$ independent variables. The mean of this sum is 0 and the variance of each term is bounded as we supposed $\phi^{0}$ to be smooth. The variance of $h_{L+1}$ is thus $O(L)$. We can also neglect the second term in the first line as

$$
\mathbb{E}\left[\frac{1}{L^{3}} \sum_{x=-L}^{L+1}\left(\Phi(x, 0)-h_{x}(0)\right)^{2}\right]=\frac{1}{L^{3}} \sum_{x=-L}^{L+1} \operatorname{Var}\left(h_{x}(0)\right)=O\left(L^{-1}\right)
$$

where the last equality is easy to obtain once noticed that $h_{x}$ is the sum of $(L+x)$ independent geometric variables with bounded variance.

Set

$$
A(x, s):=-q_{x}(s) \sigma\left(q_{x}(s)\right)+\eta_{x}(s) \sigma\left(q_{x}(s)\right)+q_{x}(s) \operatorname{sg}\left(\eta_{x}(s)\right)-\left(\left|\eta_{x}(s)\right|-\mid \operatorname{sg}\left(\eta_{x}(s) \mid\right) .\right.
$$

From the previous equations one gets that

$$
\mathbb{E}\left[\frac{1}{L^{3}} \sum_{x=-L+1}^{L}\left(\Phi\left(x, L^{2} t\right)-h_{x}\left(L^{2} t\right)\right)^{2}\right]=\frac{1}{L^{3}} \int_{0}^{L^{2} t} \sum_{x=-L+1}^{L} \mathbb{E}[A(s, x)] \mathrm{d} s+o(1) .
$$

To understand better the rest of the proof, the reader should notice that if $\left(\eta_{x}(s)\right)_{x \in\{-L, \ldots, L\}}$ were distributed like geometric variables it would be possible to factorize $\mathbb{E}[A(x, s)]$ in a product of negative sign and from equation (A.18) the proof would be over. Indeed, for $q>0$ and $\eta$ distributed like a geometric variable of mean $u>0$ (or $-\eta$ is distributed like a geometric variable of mean $-u>0$ ),

$$
\mathbb{E}[-q \sigma(q)+\eta \sigma(q)+q \operatorname{sg}(\eta)-(|\eta|-|\operatorname{sg}(\eta)|)]=-(q-u)(\sigma(q)-\sigma(u)) \leq 0,
$$

(recall that $\sigma(\cdot)$ is an increasing function). It is not true in general that $\eta_{x}(s)$ are geometrically distributed for $s>0$ but this is reasonable to think that their distribution is close to geometric: as the system mixes locally in finite time, what one should observe on finite but large windows is close to an equilibrium measure, and from [1] it is known that the only (infinite-volume translation invariant) equilibrium measures for the zero-range process are convex combinations of products of geometric variables. Most of our efforts will therefore be focused on proving convergence to the infinite volume measure for a space-time averaged version of the probability distribution of the $\eta_{x}(s)$ (using this space-time average is somehow crucial for the proof to work).

As the limiting object is an infinite volume measure, it is somehow more convenient to consider $\eta(s)$ as an element of $\mathbb{Z}^{\mathbb{Z}}$ by periodizing it: for the system of size $(2 L+1)$ one sets $\eta_{x+k(2 L+1)}=\eta_{x}$ for every $k \in \mathbb{Z}, x \in\{-L, \ldots, L\}$. For $y \in \mathbb{Z}$ one defines $\theta_{y}$ to be the shift operator $\eta \mapsto \theta_{x} \eta$ defined by

$$
\forall x \in \mathbb{Z}, \quad\left(\theta_{y} \eta\right)_{x}:=\eta_{x+y} .
$$

We define for each $L>0$ the measure $\mu_{t}^{L}$ on $\mathbb{Z}^{\mathbb{Z}}$ our space-time averaged measures by its action on local functions (for $K \in \mathbb{N}$ we call $f(\eta)$ a $K$-local function if $f$ is bounded and can be 
written as a function of $\eta_{\mid[-K, K]} ; f$ is a local function if there exists a $K$ such that it is $K$-local):

$$
\mu_{t}^{L}(f):=\mathbb{E}\left[\frac{1}{t L^{2}} \frac{1}{2 L+1} \int_{0}^{L^{2} t} \sum_{y=-L}^{L} f\left(\theta_{y}(\eta(s))\right) \mathrm{d} s\right],
$$

We want to prove that any limit point (when $L \rightarrow \infty$ ) of $\mu_{t}^{L}$ is an equilibrium and use this information to bound the right-hand side of (A.18).

We introduce some notation to describe the limiting measure. For $u \in \mathbb{R}$ define $\rho^{u}$ to be a measure on $\eta=\left(\eta_{x}\right)_{x \in \mathbb{Z}}$ such that the $\eta_{x}$ are IID geometric variables of mean $u$ if $u \geq 0$ while the $-\eta_{x}$ are IID geometric variables of mean $-u$ if $u<0$. If $\nu$ is a probability measure on $\mathbb{R}$ define

$$
\rho^{\nu}:=\int \rho^{u} \nu(\mathrm{d} u)
$$

Proposition A.4. Fix $t>0$. For any subsequence of $\left(\mu_{t}^{L_{n}}\right)_{n \geq 0}$, it is possible to find a subsubsequence $\left(\mu_{t}^{L_{n}^{\prime}}\right)_{n \geq 0}$ that converges locally to $\rho^{\nu}$ with $\nu$ a probability measure on $\mathbb{R}$ with support included in $\left[-\left\|\partial_{x} \phi^{0}\right\|_{\infty},\left\|\partial_{x} \phi^{0}\right\|_{\infty}\right]$, in the sense that for any local function $f$

$$
\lim _{n \rightarrow \infty} \mu_{t}^{L_{n}^{\prime}}(f)=\rho^{\nu}(f) .
$$

As a consequence for any local function $f$

$$
\limsup _{L \rightarrow \infty} \mu_{t}^{L}(f) \leq \max _{u \in\left[-\left\|\partial_{x} \phi^{0}\right\|_{\infty},\left\|\partial_{x} \phi^{0}\right\|_{\infty}\right]} \rho^{u}(f) .
$$

Remark A.5. Note that the convergence does not hold in the topology induced by the total variation distance: indeed $\mu_{t}^{L}$ give mass one to $L$-periodic $\eta$ whereas these configurations have mass zero for the limiting measure.

Proof of Proposition A.4. For any fixed $K>0$, the sequence of laws of $\left(\eta_{x}\right)_{x \in[-K, K]}$ under $\mu_{t}^{L_{n}}$ is tight by Lemma A.3 (iii) and hence we can extract a converging subsequence. By diagonal extraction it is possible to extract a subsequence $L_{n}^{\prime}$ of $L_{n}$ and a family of measures $\left(\mu_{K}\right)_{K} \geqslant 0$ on $\mathbb{Z}^{[-K, K]}$ such that the law of $\left(\eta_{x}\right)_{x \in[-K, K]}$ under $\mu_{t}^{L_{n}}$ converges to $\mu^{K}$ for all $K$. By construction for $H \geqslant K, \mu_{H}$ projected on $\mathbb{Z}^{[-K, K]}$ is equal to $\mu_{K}$ and by Kolmogorov extension theorem, there exists a measure $\mu$ on $\mathbb{Z}^{\mathbb{Z}}$ such that $\mu$ projected on $\mathbb{Z}^{[-K, K]}$ equals $\mu_{K}$ for all $K$. One has therefore for all local function $f$

$$
\lim _{n \rightarrow \infty} \mu_{t}^{L_{n}^{\prime}}(f)=\mu(f) .
$$

We have to show that $\mu$ can be written as $\rho^{\nu}$. First one remarks that $\mu_{t}^{L_{n}}$ is translation invariant, so that $\mu$ is too. A second point to make is that $\mu$-almost surely all the $\eta_{x}$ (that are not equal to zero) have the same sign. Indeed

$$
\begin{aligned}
\mu\left(\exists x, x^{\prime} \in \mathbb{Z}, \eta_{x} \eta_{x}^{\prime}<0\right)=\lim _{K \rightarrow \infty} \mu(\exists x, & \left.x^{\prime} \in[-K, K], \eta_{x} \eta_{x}^{\prime}<0\right) \\
& =\lim _{K \rightarrow \infty} \lim _{n \rightarrow \infty} \mu_{t}^{L_{n}^{\prime}}\left(\exists x, x^{\prime} \in[-K, K], \eta_{x} \eta_{x}^{\prime}<0\right)
\end{aligned}
$$

and

$$
\begin{aligned}
\mu_{t}^{L}\left(\exists x, x^{\prime}\right. & \left.\in[-K, K], \eta_{x} \eta_{x}^{\prime}<0\right) \\
& =\frac{1}{t L^{2}(2 L+1)} \int_{0}^{L^{2} t} \sum_{y=-L}^{L} \mathbb{P}\left[\exists x, x^{\prime} \in[-K+y, K+y], \eta_{x}(s) \eta_{x^{\prime}}(s)<0\right] \mathrm{d} s .
\end{aligned}
$$


One realizes easily that

$$
\sum_{y=-L}^{L} \mathbf{1}_{\left\{\exists x, x^{\prime} \in[-K+y, K+y], \eta_{x} \eta_{x^{\prime}}<0\right\}}
$$

is upper bounded by $(2 K+1)$ times the number of changes of sign in $\left(\eta_{x}\right)_{x \in[-L, L+1]}$. From the definition of the dynamics, a transition can only lower the number of changes of sign. Its initial value is smaller than the number of changes of monotonicity of $\phi^{0}$ (which is assumed to be finite) plus one (the "plus one" can come from periodizing). Therefore

$$
\sum_{y=-L}^{L} \mathbb{P}\left[\exists x, x^{\prime} \in[-K+y, K+y], \eta_{x}(s) \eta_{x^{\prime}}(s)<0\right] \leq 2 K C\left(\phi^{0}\right) .
$$

A third point is to show is that $\mu$ is an invariant measure for the infinite volume dynamics (the infinite volume version of (A.3), call its generator $\mathcal{L}^{\infty}$ ). For $f$ a $K$-local function one has (for $L \geq K$ large enough)

$$
\mu_{t}^{L}\left(\mathcal{L}^{\infty} f\right)=\frac{1}{t L^{2}} \frac{1}{2 L+1} \int_{0}^{L^{2} t} \sum_{y=-L}^{L} \mathbb{E}\left(\mathcal{L}^{\infty}\left(f \circ \theta_{y}\right)(\eta(s))\right) \mathrm{d} s .
$$

For $y \in[-L+K, L-K]$ the infinite volume generator applied to $f$ has the same effect as the finite volume generator so that

$$
\int_{0}^{t} \mathbb{E}\left[\mathcal{L}^{\infty}\left(f \circ \theta_{y}\right)(\eta(s))\right] \mathrm{d} s=\int_{0}^{t} \partial_{s} \mathbb{E}\left[\left(f \circ \theta_{y}\right)(\eta(s))\right] \mathrm{d} s=\mathbb{E}\left[\left(f \circ \theta_{y}\right)(\eta(t))-\left(f \circ \theta_{y}\right)(\eta(0))\right] .
$$

Therefore

$$
\begin{aligned}
& \mu_{t}^{L}\left(\mathcal{L}^{\infty} f\right)=\frac{1}{t L^{2}} \frac{1}{2 L+1} \sum_{y=-L+K}^{L-K} \mathbb{E}\left[\left(f \circ \theta_{y}\right)\left(\eta\left(t L^{2}\right)\right)-\left(f \circ \theta_{y}\right)(\eta(0))\right] \\
&+\frac{1}{t L^{2}} \frac{1}{2 L+1} \int_{0}^{L^{2} t}\left(\sum_{y=-L}^{-L+K-1}+\sum_{y=L-K+1}^{L}\right) \mathbb{E}\left(\mathcal{L}^{\infty}\left(f \circ \theta_{y}\right)(\eta(s))\right) \mathrm{d} s=O(1 / L) .
\end{aligned}
$$

As a consequence, for any local function

$$
\mu\left(\mathcal{L}^{\infty} f\right)=\lim _{n \rightarrow \infty} \mu_{t}^{L_{n}^{\prime}}\left(\mathcal{L}^{\infty} f\right)=0 .
$$

Restricted on the event $\eta_{x}$ have all the same sign, $\mathcal{L}^{\infty}$ is the generator of the zero-range process with one type of particle and therefore $\mu$ is a translation invariant measure for the zero-range process. From [1, Theorem 1.9] one can write $\mu=\rho^{\nu}$ for some $\nu$. By Lemma A.3 (iii) - (iv), under $\mu$, at time zero $\eta$ is dominated by a IID family of geometric variables of mean $\left\|\partial_{x} \phi^{0}\right\|_{\infty}$ and so is $-\eta$. This implies the claim on the support of $\nu$.

The second point of Proposition A.4 is standard; we include its proof for completeness. Given a local $f$ one can extract a subsequence $L_{n}$ such that

$$
\lim _{n \rightarrow \infty} \mu_{t}^{L_{n}}(f)=\limsup _{L \rightarrow \infty} \mu_{t}^{L}(f) .
$$

From $L_{n}$ one can extract a subsequence $L_{n}^{\prime}$ such that $\mu_{t}^{L_{n}^{\prime}}$ converges to $\rho^{\nu}$ so that

$$
\lim _{n \rightarrow \infty} \mu_{t}^{L_{n}}(f)=\lim _{n \rightarrow \infty} \mu_{t}^{L_{n}^{\prime}}(f)=\int \rho^{u}(f) \nu(\mathrm{d} u),
$$


which ends the proof.

Fix $l$ to be a large fixed integer. Set for $y \in \mathbb{Z}$

$$
B_{y}:=\{1+y, \ldots, l+y\} .
$$

For notational convenience, similarly to $\eta$ with (A.20), one considers now periodized version $\left(q_{x}(s)\right)_{s \in \mathbb{Z}}$ of $q(s)$ and $(A(x, s))_{x \in \mathbb{Z}}$ of $A(\cdot, s)$.

Now, one uses Proposition A.4 to control each term in $\mathbb{E} \sum A(s, x)$.

\section{Lemma A.6.}

$$
\lim _{l \rightarrow \infty} \limsup _{L \rightarrow \infty} \frac{1}{L^{3}} \mathbb{E} \int_{0}^{L^{2} t} \sum_{y=-L}^{L}\left|\left(\frac{1}{l} \sum_{x \in B_{y}} q_{x}(s) \operatorname{sg}\left(\eta_{x}(s)\right)\right)-q_{y}(s) \sigma\left(\frac{1}{l} \sum_{x \in B_{y}} \eta_{x}(s)\right) \mathrm{d} s\right|=0 .
$$

Proof of Lemma A.6. Fix $l>0$. For $L$ large enough, any all $y \in\{-L, \ldots, L-l\}$,

$$
\begin{aligned}
& \left|\left(\frac{1}{l} \sum_{x \in B_{y}} q_{x}(s) \operatorname{sg}\left(\eta_{x}(s)\right)\right)-q_{y}(s) \sigma\left(\frac{1}{l} \sum_{x \in B_{y}} \eta_{x}(s)\right)\right| \\
& \leq\left|q_{y}(s)\right|\left|\left(\frac{1}{l} \sum_{x \in B_{y}} \operatorname{sg}\left(\eta_{x}(s)\right)\right)-\sigma\left(\frac{1}{l} \sum_{x \in B_{y}} \eta_{x}(s)\right)\right|+\max _{x \in B_{y}}\left|q_{x}(s)-q_{y}(s)\right| .
\end{aligned}
$$

Moreover, uniformly in $y \in\{-L, \ldots, L-l\}$, as a consequence of Lemma A.3 (ii)

$$
\max _{y \in\{-L, \ldots, L-l\}, x \in B_{y}, s \geq 0}\left|q_{x}(s)-q_{y}(s)\right|=O(l / L) .
$$

The contribution of $y \in\{L-l+1, L\}$ to the sum under the integral in (A.37) is $O(l)$. Therefore summing over $y \in\{-L, \ldots, L\}$, integrating over $s$ and taking expectation one gets

$$
\begin{aligned}
& \left|\int_{0}^{t L^{2}} \mathbb{E}\left[\sum_{y=-L}^{L}\left(\frac{1}{l} \sum_{x \in B_{y}} q_{x}(s) \operatorname{sg}\left(\eta_{x}(s)\right)\right)-q_{y}(s) \sigma\left(\frac{1}{l} \sum_{x \in B_{y}} \eta_{x}(s)\right)\right]\right| \\
& \leq\left(\max _{y}\left|q_{y}(s)\right|\right)\left|\int_{0}^{t L^{2}} \mathbb{E}\left[\sum_{y=-L}^{L}\left(\frac{1}{l} \sum_{x \in B_{y}} \operatorname{sg}\left(\eta_{x}(s)\right)\right)-\sigma\left(\frac{1}{l} \sum_{x \in B_{y}} \eta_{x}(s)\right)\right]\right|+O\left(l L^{2}\right) \\
& \quad=\left(\max _{y}\left|q_{y}(s)\right|\right) t L^{2}(2 L+1) \mu_{t}^{L}\left(\left|\left(\frac{1}{l} \sum_{x \in B_{0}} \operatorname{sg}\left(\eta_{x}\right)\right)-\sigma\left(\frac{1}{l} \sum_{x \in B_{0}} \eta_{x}\right)\right|\right)+O\left(l L^{2}\right)
\end{aligned}
$$

where $\mu_{t}^{L}$ is defined in (A.21). Therefore, the proof of our statement is finished provided one proves

$$
\lim _{l \rightarrow \infty} \limsup _{L \rightarrow \infty} \mu_{t}^{L}\left(\left|\left(\frac{1}{l} \sum_{x \in B_{0}} \operatorname{sg}\left(\eta_{x}\right)\right)-\sigma\left(\frac{1}{l} \sum_{x \in B_{0}} \eta_{x}\right)\right|\right)=0 .
$$


From Proposition A.4 one has

$$
\begin{aligned}
\limsup _{L \rightarrow \infty} \mu_{t}^{L}\left(\mid\left(\frac{1}{l} \sum_{x \in B_{0}} \operatorname{sg}\left(\eta_{x}\right)\right)\right. & \left.-\sigma\left(\frac{1}{l} \sum_{x \in B_{0}} \eta_{x}\right) \mid\right) \\
\leq & \sup _{0 \leq u \leq\left\|\partial_{x} \phi^{0}\right\|_{\infty}} \rho^{u}\left(\left|\left(\frac{1}{l} \sum_{x \in B_{0}} \operatorname{sg}\left(\eta_{x}\right)\right)-\sigma\left(\frac{1}{l} \sum_{x \in B_{0}} \eta_{x}\right)\right|\right)
\end{aligned}
$$

and one can check that the right-hand side term tends to zero when $l$ tends to infinity: we note that under $\rho^{u}$, for every $x$ one has $\rho^{u}\left(\operatorname{sg}\left(\eta_{x}\right)\right)=\sigma(u)$, and the law of large numbers tell us that the two terms $\frac{1}{l} \sum_{x \in B_{y}} \operatorname{sg}\left(\eta_{x}\right)$ and $\sigma\left(\frac{1}{l} \sum_{x \in B_{y}} \eta_{x}\right)$ have the same limit when $l$ tends to infinity. However, because of the sup over $u$ one needs more quantitative estimates than the law of large numbers to conclude. For instance we can get them by the use of second moment method; we leave the details to the reader.

Similarly to Lemma A.6 one shows that

\section{Lemma A.7.}

$$
\lim _{l \rightarrow \infty} \limsup _{L \rightarrow \infty} \frac{1}{L^{3}} \int_{0}^{L^{2} t} \sum_{y=-L}^{L} \mathbb{E}(G(\eta(s)))=\lim _{l \rightarrow \infty} \limsup _{L \rightarrow \infty} \frac{t(2 L+1)}{L} \mu_{t}^{L}(G(\eta))=0
$$

where

$$
G(\eta)=\mid\left(\frac{1}{l} \sum_{x \in B_{y}}\left|\eta_{x}(s)\right|-\left|\operatorname{sg}\left(\eta_{x}(s) \mid\right)-\left(\frac{1}{l} \sum_{x \in B_{y}} \eta_{x}(s)\right) \sigma\left(\frac{1}{l} \sum_{x \in B_{y}} \eta_{x}(s)\right)\right| .\right.
$$

Proof. The proof is very similar to that of Proposition A.6, the only additional technical point being that the function $G(\eta)$ is not bounded so that one cannot use directly Proposition A.4. However stochastic domination given by Lemma A.3 (iii) allows us to get the same conclusion by considering the function $\eta \mapsto \min (G(\eta), K)$, and letting $K$ tend to infinity afterwards. Altogether one gets

$$
\limsup _{L \rightarrow \infty} \mu_{t}^{L}(G) \leq \sup _{0 \leq u \leq\left\|\partial_{x} \phi^{0}\right\|_{\infty}} \rho^{u}(G)
$$

We end the proof in the same way that for the previous Lemma remarking that

$$
\rho^{u}\left(\left|\eta_{x}\right|-\left|\operatorname{sg}\left(\eta_{x}\right)\right|\right)=u \sigma(u)
$$


Now we are ready to conclude:

$$
\begin{gathered}
\sum_{y=-L}^{L} A(y, s) \\
=\sum_{y=-L}^{L}\left\{-q_{y}(s) \sigma\left(q_{y}(s)\right)+\frac{1}{l}\left(\sum_{x \in B_{y}} \eta_{x}(s) \sigma\left(q_{x}(s)\right)+q_{x}(s) \operatorname{sg}\left(\eta_{x}(s)\right)-\left(\left|\eta_{x}(s)\right|-\mid \operatorname{sg}\left(\eta_{x}(s) \mid\right)\right)\right\}\right. \\
\leq \sum_{y=-L}^{L}-q_{y}(s) \sigma\left(q_{y}(s)+\left(\frac{1}{l} \sum_{x \in B_{y}} \eta_{x}(s)\right) \sigma\left(q_{y}(s)\right)+q_{y}(s) \sigma\left(\frac{1}{l} \sum_{x \in B_{y}} \eta_{x}(s)\right)\right. \\
-\left(\frac{1}{l} \sum_{x \in B_{y}} \eta_{x}(s)\right) \sigma\left(\frac{1}{l} \sum_{x \in B_{y}} \eta_{x}(s)\right)+R(s, l, L) \\
=R(s, l, L)-\sum_{y=-L}^{L}\left[q_{y}(s)-\left(\frac{1}{l} \sum_{x \in B_{y}} \eta_{x}(s)\right)\right]\left[\sigma\left(q_{y}(s)\right)-\sigma\left(\frac{1}{l} \sum_{x \in B_{y}} \eta_{x}(s)\right)\right] \quad(\mathrm{A} .46)
\end{gathered}
$$

where

$$
\begin{aligned}
R(s, l, L)=- & \sum_{y=-L}^{L} \frac{1}{l}\left(\sum_{x \in B_{y}} \eta_{x}(s)\left(\sigma\left(q_{y}(s)\right)-\sigma\left(q_{x}(s)\right)\right)\right) \\
+ & \left|\left(\frac{1}{l} \sum_{x \in B_{y}} q_{x}(s) \operatorname{sg}\left(\eta_{x}(s)\right)\right)-q_{y}(s) \sigma\left(\frac{1}{l} \sum_{x \in B_{y}} \eta_{x}(s)\right)\right| \\
+ & \mid\left(\frac{1}{l} \sum_{x \in B_{y}}\left|\eta_{x}(s)\right|-\left|\operatorname{sg}\left(\eta_{x}(s) \mid\right)-\left(\frac{1}{l} \sum_{x \in B_{y}} \eta_{x}(s)\right) \sigma\left(\frac{1}{l} \sum_{x \in B_{y}} \eta_{x}(s)\right)\right|\right.
\end{aligned}
$$

and the second term is non positive ( $a-b$ and $\sigma(a)-\sigma(b)$ have the same sign).

According to $(i i)-(i i i)$ in Lemma A.3 (to control the first term) and Lemmata A.6 and A.7

$$
\lim _{l \rightarrow \infty} \limsup _{L \rightarrow 0} \frac{1}{L^{3}} \int_{0}^{L^{2} t} \mathbb{E} R(s, l, L) \mathrm{d} s=0 .
$$

This implies

$$
\limsup _{L \rightarrow \infty} \frac{1}{L^{3}} \int_{0}^{L^{2} t} \sum_{y=-L}^{L} \mathbb{E} A(x, s) \mathrm{d} s \leq 0
$$

and therefore the result that we want to prove, from (A.18).

A.4. Concluding the proof of Theorem 3.4. It is not hard to transform the $\mathbb{L}_{2}$ statement of Proposition A.2 into the desired "almost sure" statement:

Proposition A.8. For any $\varepsilon>0, t \geq 0$, w.h.p

$$
\max _{x \in\{-L, \ldots, L+1\}} \frac{1}{L}\left|\Phi\left(x, L^{2} t\right)-\hat{h}_{x}\left(L^{2} t\right)\right| \leq \varepsilon .
$$


Proof. Also here we write $h$ for $\hat{h}$. Note that from Lemma A.3 $(i i i)$ the random vector $\left(\left|\eta_{x}(t)\right|\right)_{x \in\{-L, \ldots, L\}}$ is stochastically dominated for every $t$ by a vector of IID time-independent geometric variables. This implies that there exists a constant $C$ such that for any $t \geq 0$, w.h.p.

$$
\left|h_{x}(t)-h_{y}(t)\right| \leq C|x-y| \text { for every } x, y \in\{-L, \ldots, L\},|x-y| \geq \log L
$$

(this can be proved by using large deviation estimates and a union bound on $x, y \in\{-L, \ldots, L\}$ ). Moreover Lemma A.3 $(i)$ ensures that $\Phi(\cdot, t)$ is always Lipschitz so that (A.51) holds also for $\Phi(\cdot, t)-h .(t)$.

With (A.51) and $L$ large enough, one has

$$
\begin{gathered}
\left\{\max _{x \in\{-L, \ldots, L+1\}}\left|\Phi\left(x, L^{2} t\right)-h_{x}\left(L^{2} t\right)\right| \geq \varepsilon L\right\} \\
\Longrightarrow\left\{\sum_{x \in\{-L, \ldots, L+1\}}\left|\Phi\left(x, L^{2} t\right)-h_{x}\left(L^{2} t\right)\right|^{2} \geq \frac{\varepsilon^{3} L^{3}}{10 C}\right\}
\end{gathered}
$$

so that the left-hand side event has small probability when $L$ is large, otherwise Proposition A.2 would be false.

A.5. Laplacian bounds. Recall that $\Phi(x, t)$ is the solution of the Cauchy problem (3.12). We want to bound $\Phi(x, t)$ above and below with the solution of a suitable heat equation. For this, we will suppose that the function $\phi^{0}$, through which the initial condition $\Phi_{0}$ for $\Phi(x, t)$ is defined, is concave on $[-1,1]$ (in addition to the assumptions required for Theorem 3.4). One defines the evolution $\Phi_{1}(x, t)$ as the solution of

$$
\begin{cases}\partial_{t} \Phi_{1}(x, t) & =\frac{1}{2} \Delta \Phi_{1}(x, t) \\ \Phi_{1}(-L, t) & =\Phi(L+1, t)=0 \\ \Phi_{1}(x, 0) & =\Phi_{0}(x)\end{cases}
$$

for $t \geq 0, x \in\{-L+1, L\}$. Also we define $\Phi_{2}(x, t)$ as the solution of the analogous equation (with the same boundary values) where the discrete Laplacian is multiplied by $(1 / 2) \sigma^{\prime}\left(\left\|\partial_{x} \phi^{0}\right\|_{\infty}\right)=$ $1 /\left(1+\left\|\partial_{x} \phi^{0}\right\|_{\infty}\right)^{2}$.

Proposition A.9. For every $t \geq 0$ every $x \in\{-L, \ldots, L+1\}$ one has

$$
\Phi_{1}(x, t) \leq \Phi(x, t) \leq \Phi_{2}(x, t) .
$$

Proof. We prove the upper bound, the lower one being very similar. Suppose that the result does not hold and set

$$
T:=\max \left\{t \mid \Phi(x, t) \leq \Phi_{2}(x, t) \text { for every } t \leq T, x \in\{-L, \ldots, L+1\}\right\} .
$$

Note that by property of the heat-equation, $\Phi_{2}(x, t)$ is a strictly concave function of $x$ for all positive $t$ (except in the case where one starts from the flat initial condition but in that case the statement is trivial). Let $x_{0}$ be such that

$$
\Phi\left(x_{0}, T\right)=\Phi_{2}\left(x_{0}, T\right) .
$$

Then one remarks that $q_{x_{0}}(T)-q_{x_{0}-1}(T)<0$ (by strict concavity of $\Phi_{2}(\cdot, T)$ ) and that by Lemma A.3 $\max _{x}\left|q_{x}(t)\right| \leq\left\|\partial_{x} \phi^{0}\right\|_{\infty}$ so that

$$
\sigma\left(q_{x_{0}}(T)\right)-\sigma\left(q_{x_{0}-1}(T)\right)<\left(q_{x_{0}}(T)-q_{x_{0}-1}(T)\right) \sigma^{\prime}\left(\left\|\partial_{x} \phi_{0}\right\|_{\infty}\right)
$$


(since $\sigma^{\prime}(\cdot)$ is decreasing on $\mathbb{R}^{+}$) and hence

$$
\begin{aligned}
2 \partial_{t}\left[\Phi_{2}-\Phi\right] & \left(x_{0}, T\right)=\sigma^{\prime}\left(\left\|\partial_{x} \phi_{0}\right\|_{\infty}\right) \Delta \Phi_{2}(x, t)-\sigma\left(q_{x_{0}}(T)\right)+\sigma\left(q_{x_{0}-1}(T)\right) \\
& >\sigma^{\prime}\left(\left\|\partial_{x} \phi_{0}\right\|_{\infty}\right)\left[\left(\Phi_{2}(x+1, t)+\Phi_{2}(x-1, t)\right)-(\Phi(x+1, t)+\Phi(x-1, t))\right] .
\end{aligned}
$$

Since the last expression is non-negative, one has $\Phi(x, t)<\Phi_{2}(x, t)$ on an interval $[T, T+\varepsilon(x)]$ for some $\varepsilon(x)>0$, for every $x \in\{-L, \ldots, L+1\}$ and that concludes the proof since the only possibility is that $T=\infty$.

\section{ACKNOWLEDGMENTS}

The authors would like to thank P. Cardaliaguet for enlightening discussions and valuable comments on various analytical aspects of the present work. During part of the writing, H.L. was hosted by Instituto Nacional de Matemática Pura e Aplicada; he acknowledges kind hospitality and support.

\section{REFERENCES}

[1] E. D. Andjel, Invariant measure for the zero-range process, Ann. Probab. 10 (1982), 525-547.

[2] P. Caputo, F. Martinelli, F. Simenhaus and F. L. Toninelli, "Zero" temperature stochastic 3D Ising model and Dimer covering fluctuation: a first step towards mean curvature motion, Comm. Pure Appl. Math. 64 (2011), 778-831.

[3] R. Cerf, S. Louhichi, The initial drift of a $2 D$ droplet at zero temperature, Probab. Theory Relat. Fields 137 (2007), 379-428.

[4] L. Chayes, R. H. Schonmann and G. Swindle, Lifshitz' Law for the Volume of a 2-Dimensional Droplet at Zero Temperature, J. Stat. Phys. 79 (1995) 821-831

[5] A. De Masi, E. Orlandi, E. Presutti, L. Triolo, Motion by curvature by scaling nonlocal evolution equations, J. Statist. Phys. 73 (1993), 543-570.

[6] A. De Masi, E. Orlandi, E. Presutti, L. Triolo, Glauber evolution with the Kac potentials. I. Mesoscopic and macroscopic limits, interface dynamics, Nonlinearity 7 (1994), 633-696.

[7] P. Diaconis and M. Shahshahani, Generating a random permutation with random transpositions, Z. Wahrsch. Verw. Gebiete 57 (1981), 159-179.

[8] H. G. Eggleston, Convexity, Cambridge University Press, New York 1958

[9] L. R. Fontes, R. H. Schonmann and V. Sidoravicius, Stretched exponential fixation in stochastic Ising models at zero temperature, Comm. Math. Phys. 228 (2002), 495-518.

[10] T. Funaki and H. Spohn, Motion by Mean Curvature from the Ginzburg-Landau $\nabla \phi$ Interface Model, Comm. Math. Phys. 185 (1997), 1-36.

[11] M. E. Gage, Evolving plane curvature in relative geometries I, Duke Math. J. 74 (1993) 441-466.

[12] M. E. Gage and Y. Li, Evolving plane curvature in relative geometries II, Duke Math. J. 75 (1994) $79-98$.

[13] M. E. Gage and R. S. Hamilton, The heat equation shrinking of convex plane curves, J. Diff. Geom. 23 (1986), 69-96.

[14] M. A. Grayson, The heat equation shrinks embedded plane curves to round points, J. Differential Geometry 26 (1986), 285-314.

[15] M. A. Katsoulakis and P. E. Souganidis, Stochastic Ising models and anisotropic front propagation, J. Statist. Phys. 87 (1997), 63-89.

[16] C. Kipnis and C. Landim, Scaling limits of interacting particle systems, Springer-Verlag, Berlin, 1999.

[17] P. L. Krapivsky, Limiting Shapes of Ising Droplet, Ising Finger and Ising Soliton, arXiv:1111.6209v1

[18] H. Lacoin, Approximate Lifshitz law for the zero-temperature stochastic Ising model in any dimension, (preprint) arXiv:1102.3466 [math-ph].

[19] D. Levin, Y. Peres and E. Wilmer, Markov Chains and Mixing Times, American Mathematical Society, Providence, RI, (2009).

[20] I. M. Lifshitz, Kinetics of ordering during second order phase transitions, Soviet Physics JETP 15 (1962), 939-942.

[21] T. M. Liggett, Interacting Particle Systems, Springer-Verlag, New York, 1985

[22] E. Lubetzky, F. Martinelli, A. Sly and F. L. Toninelli, Quasi-polynomial mixing of the 2D stochastic Ising model with "plus" boundary up to criticality, to appear on J. Eur. Math. Soc. 
[23] H. Rost, Nonequilibrium behaviour of a many particle process: density profile and local equilibria, Z. Wahrsch. Verw. Gebiete 58 (1981), 41-53.

[24] H. Spohn, Interface motion in models with stochastic dynamics, J. Stat. Phys. 71 (1993), 1081-1132.

[25] D. B. Wilson Mixing times of Lozenge tiling and card shuffling Markov chains, Ann. Appl. Probab. 14 (2004) $274-325$.

H. Lacoin, CEREMAde - UMR CNRS 7534 - Université Paris Dauphine, Place du Maréchal de LATtre de TASsigny, 75775 CEDEX-16 PARIs, France.

E-MAIL: lacoin@ceremade.dauphine.fr

F. Simenhaus, Ceremade - Université Paris Dauphine - UMr CNRS 7534, Place du Maréchal DE Lattre de TAssigny, 75775 CEDEX-16 PARis France.

E-MAIL: simenhaus@ceremade.dauphine.fr

F. L. Toninelli, Université de Lyon and CNRS, Ecole Normale Superieure de Lyon, Laboratoire De Physique, 46 Allée d'Italie, 69364 Lyon, France.

E-MAIL: fabio-lucio.toninelli@ens-lyon.fr 